\title{
Um modelo de pressão arterial sistêmica utilizando equações diferenciais com impulso
}

\author{
Sergio Augusto Malacrida Junior \\ DISSERTAÇÃO APRESENTADA \\ $\mathrm{AO}$ \\ INSTITUTO DE MATEMÁTICA E ESTATÍSTICA \\ DA \\ UNIVERSIDADE DE SÃO PAULO \\ PARA \\ OBTENÇÃO DO GRAU DE MESTRE \\ EM \\ CIÊNCIAS \\ Área de Concentração: Matemática \\ Orientador Prof. Dr. Luciano Barbanti
}

São Paulo, abril de 2007. 


\title{
Um modelo de pressão arterial sistêmica utilizando \\ equações diferenciais com impulso
}

\author{
Este exemplar corresponde à redação final da \\ dissertação devidamente corrigida e defendida \\ por Sergio Augusto Malacrida Junior \\ e aprovada pela Comissão Julgadora.
}

São Paulo, 9 de abril de 2007.

Banca Examinadora:

Prof. Dr. Luciano Barbanti (orientador) - IME/USP

Prof. Dr. Bruno Caramelli - FM/USP

Prof. Dr. Pedro Tonelli - IME/USP 
Mara, Pedrinho, Rafa, Lúcia e Gó 


\section{Agradecimentos}

Esse trabalho teria sido impossível sem a co-orientação de João Carlos Prandini. Agradeço a ele também pela amizade e pelas conversas que tivemos ao longo dos mais de cinco em que nos conhecemos.

Agradeço muito também a Luciano Barbanti, orientador e amigo, que me incentivou e apoiou incondicionalmente o meu sonho de estudar Matemática.

Aproveito ainda para agradecer aos amigos Marcelo Rabbat, Ricardo Pires, Roberto Masaishi, Guilherme Benites e Rodrigo De Losso que me estimularam a continuar estudando Matemática.

Por fim, desculpo-me com minha família, de modo especial com Mara, Pedrinho e Rafa, pela ausência, apesar de saber que a compreensão, o amor e a paciência que eles têm comigo são infinitas. 


\title{
Resumo
}

As Equações Diferenciais com Impulso (EDIs) têm aplicações em diversos ramos do conhecimento, indo desde a mensuração de riscos (de mercado, de crédito, etc) até a Física e a Medicina. Neste trabalho, as EDIs são inicialmente definidas e são apresentados alguns exemplos. A seguir é feito um estudo acerca da existência, periodicidade, quase-periodicidade, estabilidade e outras propriedades das soluções das EDIs. Finalmente, é feita uma aplicação em Medicina, em particular na análise da pressão cardíaca. São apresentados alguns modelos para a Pressão Arterial Sangüínea (PAS) e dentre eles destaca-se o modelo que utiliza as EDIs, uma vez que os batimentos cardíacos podem ser entendidas como impulsos.

\begin{abstract}
The Impulsive Differential Equations (IDEs) have applications in several branches of the knowledge, from risk measurement (market, credit, etc) to Physics and the Medicine. In this work, the IDEs are initially defined and a few examples are presented. After this, we study, among other properties, the existence, periodicity, almost - periodicity and stability of the IDE solutions. Finally, an application to Medicine is presented, dealing particularly with the analysis of blood pressure. A few models for Systemic Arterial Pressure (SAP) are presented, and amongst them, we exhibit models based on IDEs, as heart beats can be thought of as impulses.
\end{abstract}




\section{Introdução}

A descrição matemática da evolução de alguns fenômenos naturais que sofrem perturbações instantâneas merece um tratamento adequado. Para tal idealização, o estudo de sistemas dinâmicos com trajetórias descontínuas é uma escolha natural. Alguns desses sistemas são chamados de Equações Diferenciais com Impulso (EDIs).

As EDIs são utilizadas desde os anos 20 do século anterior na Física, mas o estudo de tais equações tem crescido especialmente por causa suas aplicações em Biologia, no estudo de populações que sofrem uma perda abrupta de indivíduos, como no caso da criação de peixes, em que de tempos em tempos parte da população é retirada abruptamente. Na Física, como na condução de ônibus espaciais que têm trajetória com variação súbita e, em particular, na Medicina. Há, também, aplicações em mensuração de riscos financeiros (especialmente o risco de mercado e o risco de crédito) ${ }^{1}$.

Uma das principais funções do coração é bombear sangue. O sangue carrega oxigênio dos pulmões para os vários tecidos e carrega dióxido de carbono dos tecidos de volta para o pulmão. Forma-se, portanto, um circuito fechado. O sangue carregado de dióxido de carbono é oxigenado e levado ao coração que impulsiona o sangue carregado de oxigênio para os tecidos.

A idéia de considerar a circulação sangüínea em seres humanos como um processo dinâmico foi tratada em Hoppensteadt e Peskin [3] e Simon [4]. Nos dois casos são descritos modelos de Pressão Arterial Sangüínea (PAS) usando equações diferenciais ordinárias (EDOs). O coração, como já foi dito, funciona como uma bomba que impulsiona o sangue para as artérias em determinados instantes de tempo. Isso gera descontinuidades no processo evolutivo que não são capturadas pela teoria de equações diferenciais ordinárias (EDOs). Assim, é natural modelarmos o batimento cardíaco utilizando o instrumental oferecido pelas EDIs.

Além de modelarmos o batimento cardíaco, é interessante observarmos algumas anomalias no coração e como o modelo se comporta frente a essas anomalias.

No capítulo 1 apresentaremos alguns modelos de PAS, entre eles um dinâmico que utiliza EDOs, e verificaremos a necessidade das EDIs para um modelo mais realista

\footnotetext{
${ }^{1}$ Tais aplicações podem ser encontradas em Samolienko, 1995.
} 
de PAS. No capítulo 2 apresentaremos a teoria necessária de EDIs para o entendimento do modelo de PAS com EDIs. No capítulo 3 faremos uma aplicação da teoria desenvolvida no modelo de PAS e no capítulo 4 serão observadas algumas anomalias cardíacas que podem ser compreendidas pelo modelo. 


\section{Sumário}

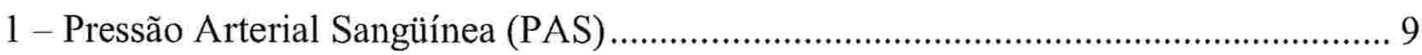

2 - Equações Diferenciais com Impulso (EDIs) .............................................................. 22

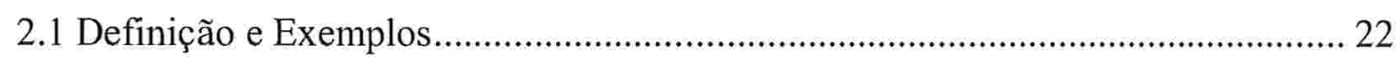

2.2 Solução de Sistemas Lineares de EDIs............................................................... 33

2.3 Oscilação, Estabilidade e Periodicidade em Sistemas de EDIs ............................ 38

3 - Aplicações das EDIs em um modelo de PAS ............................................................ 73

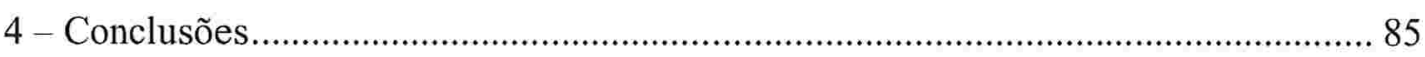

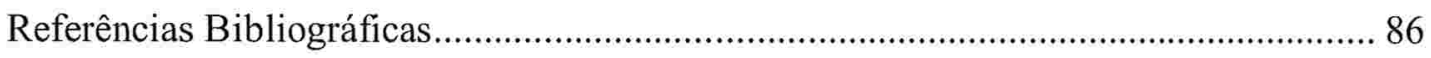




\section{1 - Pressão Arterial Sangüínea (PAS)}

Neste capítulo faremos uma descrição do modelo cardíaco desenvolvido em [3]. Um modelo para a circulação sangüínea deve tratar das seguintes variáveis: Pressão sangüínea (P), Volume de sangue em cada uma das etapas da circulação (V) e fluxo sangüíneo $(\mathrm{Q})$, entendido como o volume de sangue por minuto que passa por um determinado ponto da circulação.

O sangue carrega oxigênio dos pulmões para os vários tecidos do corpo e carrega também dióxido de carbono desses tecidos para o pulmão. Assim, a circulação forma um circuito fechado. O sangue proveniente dos órgãos e dos pulmões chega aos átrios pelas veias (direita e esquerda, respectivamente). Em seguida, é bombeado para os ventrículos, que o impulsionam por meio das artérias para os pulmões (ventrículo direito) e para os órgãos (ventrículo esquerdo).

No circuito circulatório completo, podem-se observar dois tipos distintos de circulação:

- pequena circulação (ou circulação pulmonar), em que o sangue que sai do lado direito do coração vai aos pulmões, onde absorve o oxigênio e libera o gás carbônico, retornando ao coração;

- grande circulação (ou circulação sistêmica), em que o sangue que sai do lado esquerdo do coração é distribuído a todas as partes do corpo, liberando nos tecidos o oxigênio e deles recolhendo o gás carbônico, para em seguida voltar ao coração.

Unindo as duas circulações, o trajeto geral do sangue pelo corpo é o seguinte: coração (ventrículo esquerdo) $=>$ corpo todo $=>$ retorno ao coração pelo átrio direito $=>$ ventrículo direito $=>$ pulmões $\Rightarrow>$ volta ao coração pelo átrio esquerdo $\Rightarrow>$ ventrículo esquerdo, completando uma volta inteira.

O esquema a seguir representa a circulação: 


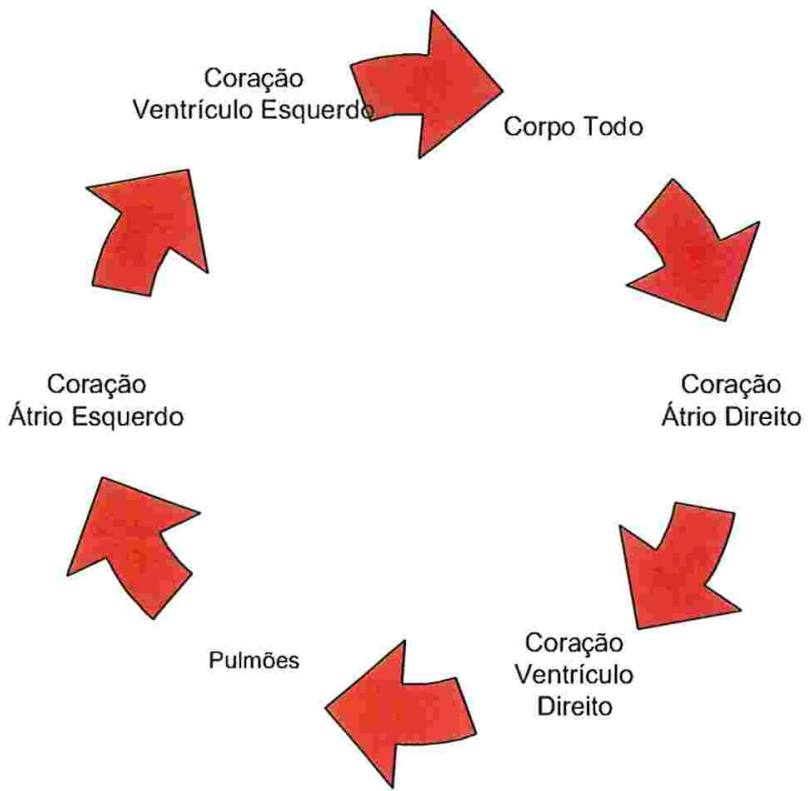

Modelo estático

As circulações sistêmicas e pulmonares funcionam de modo semelhante, com exceção ao tamanho dos circuitos, logo as equações que descreverão a circulação sistêmica terão a mesma forma das equações que descreverão a circulação pulmonar.

Os vasos por onde circulam o sangue possuem duas propriedades: resistência e complacência (compliance).

Se se supõe que um vaso é rígido, isto é que seu volume não se altera, o fluxo de sangue que passa por ele é determinado pela diferença de pressão nas extremidades de tal vaso. Fazendo a hipótese simplificadora de que a relação entre o fluxo e a diferença de pressão nas extremidades é linear, obtemos a seguinte equação:

$Q=\frac{1}{R}\left(P_{1}-P_{2}\right)$, onde

$Q$ - é o fluxo de sangue que passa pelo vaso, medido em litros/minuto,

$P_{1}, P_{2}$ - são as pressões nas extremidades dos vasos, medidas em $\mathrm{mm}$ de $\mathrm{Hg}$,

$R$ - é uma constante que chamaremos de resistência do vaso.

Por outro lado, se se supõe que o vaso não é rígido, então o volume que o vaso pode conter é ajustado até que as pressões nas extremidades sejam iguais a $P$. Desse 
modo, se denotarmos por $V$ o volume (medido em litros) do vaso e admitirmos que a relação entre $V$ e $P$ é linear, obtemos:

$V=C P \quad$ (1) , onde

$C$ - é uma constante que chamaremos de complacência do vaso.

À expressão anterior, poderíamos adicionar o volume residual do vaso $\left(V_{0}\right)$ e obter $V=V_{0}+C P$.

Todo o vaso apresenta complacência e resistência. Nos grandes vasos a complacência é mais importante, no sentido de que nesses vasos não há grande diferença de pressão nas extremidades. Por outro lado, nos vasos pequenos, em que há pouca variação de volume, a resistência é mais importante.

Em todos os modelos que consideraremos, o coração funciona como um par de bombas. O ventrículo esquerdo é equipado com uma válvula de entrada (mitral) e uma válvula de saída (aórtica). Quando o ventrículo é relaxado (diástole), a válvula mitral é aberta e a de saída é fechada. Durante esse período de tempo, o ventrículo esquerdo recebe o sangue à pressão das veias pulmonares $(5 \mathrm{~mm}$ de $\mathrm{Hg})$. Quando o ventrículo contrai (sístole), fecha-se a válvula de entrada e abre-se a de saída. O sangue é então bombeado com pressão de $100 \mathrm{~mm}$ de $\mathrm{Hg}$.

Se assumirmos a fórmula (1), o volume máximo, que ocorre no final da diástole (ED - end of diastole), é dado por:

$V_{E D}=C_{\text {cliastole }} P_{v}$, onde

$V_{E D}$ - Volume de sangue no ventrículo esquerdo imediatamente após a diástole.

$C_{\text {diastole }}$ - Coefíciente de complacência do coração na diástole.

$P_{v} \quad$ - Pressão na veia que chega no ventrículo esquerdo.

Já o volume mínimo, que ocorre no final da sístole (ES - end of systole), é dado por:

$V_{E S}=C_{\text {sistole }} P_{a}$, onde 
$V_{E S}$ - Volume de sangue no ventrículo esquerdo imediatamente após a sístole.

$C_{\text {sistole }}$ - Coeficiente de complacência do coração na sístole.

$P_{a} \quad$ - Pressão nas artérias que saem do ventrículo esquerdo.

Assim, o volume bombeado $\left(V_{B}\right)$ pelo coração a cada batida cardíaca é dado por:

$V_{B}=V_{E D}-V_{E S}$

Simplificaremos o modelo supondo que $C_{\text {sistole }}=0$ e desse modo temos que $V_{B}=C_{\text {diastole }} P_{v}$. Se denotarmos por $F$ a frequiência cardíaca (batidas/minuto), e supormos que $F$ é constante, obtemos a seguinte expressão para o fluxo Q:

$Q=F V_{B}=F C_{\text {diastole }} P_{v}$.

Definimos $K=F C_{\text {diastole }}, K$ é o coeficiente de bombeamento do ventrículo, e obtemos $Q=K P_{v}$.

A freqüência cardíaca é a mesma nos lados direito e esquerdo do coração. Apesar disso, os dois lados do coração estão conectados por diferentes sistemas de vasos. Assim, temos uma expressão para o fluxo cardíaco esquerdo e outra para o fluxo cardíaco direito:

$Q_{R}=K_{R} P_{s v} \quad$ (2) e $Q_{L}=K_{L} P_{p v} \quad$ (3), onde

$Q_{R}=$ Fluxo cardíaco do lado direito do coração,

$K_{R}=$ Coeficiente de bombeamento do lado direito do coração,

$P_{s v}=$ Pressão sistêmica venosa,

$Q_{L}=$ Fluxo cardíaco do lado esquerdo do coração,

$K_{L}=$ Coeficiente de bombeamento do lado esquerdo do coração,

$P_{p v}=$ Pressão pulmonar venosa, 
Se fizermos a hipótese de que as artérias e veias (pulmonares e sistêmicas) são vasos com complacência, obtemos as seguintes equações:

$V_{i}=C_{i} P_{i}$, onde $i=s a$ (arterial sistêmica) (4), $s v$ (venosa sistêmica) (5), pa (arterial pulmonar) (6), $p v$ (venosa pulmonar) (7).

Assumindo também que os tecidos (pulmonares e sistêmicos) possuem resistência ao fluxo sangüíneo, temos que:

$Q_{S}=\frac{1}{R_{S}}\left(P_{s a}-P_{s v}\right)(8)$

$Q_{P}=\frac{1}{R_{P}}\left(P_{p a}-P_{p v}\right)(9)$, onde

$Q_{s}$ - Fluxo sangüíneo sistêmico,

$R_{S}$ - Coeficiente de resistência sistêmica,

$Q_{P}$ - Fluxo sangüíneo pulmonar,

$R_{P}$ - Coeficiente de resistência pulmonar.

$P_{i}$ - Pressão em $i=$ sa (arterial sistêmica), sv (venosa sistêmica), pa (arterial pulmonar), pv (venosa pulmonar).

Temos assim 8 parâmetros $\left(K_{R}, K_{L}, R_{S}, R_{P}, C_{s a}, C_{s v}, C_{p a}, C_{p v}\right), 8$ equações (numeradas de (2) a (9)), e 12 incógnitas $\left(Q_{R}, Q_{L}, Q_{S}, Q_{P}, P_{s a}, P_{s v}, P_{p a}\right.$, $\left.P_{p v}, V_{s a}, V_{s v}, V_{p a}, V_{p v}\right)$. Como o volume total $\left(V_{T}\right)$ de sangue no corpo humano é dado (aproximadamente 5 litros), podemos incluir a seguinte equação:

$V_{T}=V_{s a}+V_{s v}+V_{p a}+V_{p v}(10)$ 
Se, adicionalmente, supormos que o fluxo sangüíneo é o mesmo em qualquer ponto da circulação, isto é que $Q_{R}=Q_{L}=Q_{S}=Q_{P}=Q$, ficamos com 9 equações e 9 incógnitas. A solução desse modelo é dada pelas seguintes expressões:

$$
\begin{aligned}
& Q=\frac{V_{p a}}{T_{p a}}, \\
& V_{i}=\frac{T_{i} V_{T}}{\sum_{i} T_{i}}, \\
& P_{i}=\frac{T_{i} V_{T}}{C_{i} \sum_{i} T_{i}}
\end{aligned}
$$

$i=s a$ (arterial sistêmica), $s v$ (venosa sistêmica), $p a$ (arterial pulmonar), $p v$ (venosa pulmonar);

e,

$$
T_{s v}=\frac{C_{s v}}{K_{R}}, T_{p v}=\frac{C_{p v}}{K_{L}}, T_{s a}=\frac{C_{s a}}{K_{R}}+\frac{C_{s a}}{R_{S}} \text {, e } T_{p a}=\frac{C_{p a}}{K_{L}}+\frac{C_{p a}}{R_{P}}
$$

\section{Modelo estático com mecanismo de resposta}

O modelo anterior não possui qualquer mecanismo externo de controle. O sistema nervoso, por exemplo, não age sobre tal modelo. Na realidade, a PAS é controlada por um mecanismo de resposta. Alguns dos elementos desse mecanismo são:

- Os baroreceptores, que ficam localizados em grandes vasos, na artéria caródita e no arco da aorta, são sensíveis a mudanças na pressão sangüínea. Quando há uma variação na pressão sangüínea, eles reportam essa variação ao sistema nervoso que por sua vez ajusta a freqüência cardíaca e a resistência periférica.

- Além dos baroreceptores, há receptores químicos conhecidos como quimioreceptores, que medem alterações nas concentrações de gás carbônico e oxigênio na circulação. Eles se localizam nos corpos carótidos e aórticos. Os que estão na carótida percebem decréscimos na taxa de oxigênio no sangue e os que 
estão na aorta percebem aumentos de dióxido de carbono. Como resultado dessas medidas, a frequiência respiratória é aumentada ou diminuída pelo sistema nervoso. A freqüência cardíaca ajusta-se com as mudanças na freqüência respiratória e a pressão sangüínea é assim controlada.

No modelo sem mecanismo de resposta, a freqüência cardíaca $F$ era um parâmetro externo. Agora vamos assumir que $F$ é uma incógnita. Vamos assumir também que um ajuste na freqüência cardíaca tem por objetivo levar a pressão arterial sangüínea até um determinado nível de equilíbrio que denotaremos por $P^{*}$. Isso é importante, pois uma pressão alta pode causar desgaste nos vasos, enquanto que uma pressão baixa pode causar desmaios e oxigenação inadequada dos tecidos. Assim, $P_{s a}=P^{*}$. Esse modelo, que é uma alteração do modelo anterior, será chamado de modelo cardíaco com mecanismo de resposta. Como ainda não há um elemento dinâmico no modelo, ele será denominado, juntamente com o anterior, de modelo estático. Negligenciando $P_{s v}$ na equação 8 (isso porque $P_{s v} \ll P_{s a}$ e o erro dessa aproximação nos valores obtidos é no máximo $2 \%$ ) e assumindo $V_{p v}=V_{p a}=0$, esse modelo fica definido pelas seguintes equações:

$$
\begin{aligned}
& Q=Q_{p}=Q_{s}=Q_{L}=Q_{R}=F C_{R} P_{s v}=\frac{P^{*}}{R_{s}}, \\
& V_{T}=V_{s}=V_{s a}+V_{s v}=C_{s a} P^{*}+C_{s v} P_{s v} .
\end{aligned}
$$

Dessas equações, obtemos que:

$$
\begin{aligned}
& Q=\frac{P^{*}}{R_{s}}, \\
& P_{s v}=\frac{V_{T}-C_{s a} P^{*}}{C_{s v}}, \\
& F=\frac{P^{*} C_{s v}}{R_{s} C_{R}\left(V_{T}-C_{s a} P^{*}\right)} .
\end{aligned}
$$


Note que no modelo cardíaco com mecanismo de resposta, $R_{s}$ é inversamente proporcional a $F$, isto é, se houver um aumento da resistência sistêmica a freqüência cardíaca é diminuída. Além disso, o mecanismo responsável pelo acréscimo do fluxo cardíaco é um acréscimo na freqüência cardíaca, já que a pressão e o volume são independentes de $R_{s}$.

Até agora $V, Q$ e $P$ eram constantes no que diz respeito ao tempo. Na realidade o coração injeta sangue nas artérias em bombeadas discretas. Durante a contração a pressão arterial aumenta rapidamente e cai novamente após a descontração. O resultado desse processo é chamado pulso arterial. Uma pressão arterial de 12/8 (lê-se 12 por 8) quer dizer que na sístole a pressão é de $120 \mathrm{~mm}$ de $\mathrm{Hg}$ (utiliza-se também pressão sistólica) e na diástole é de $80 \mathrm{~mm}$ de $\mathrm{Hg}$ (utiliza-se também pressão diastólica). A diferença entre esses dois valores, que nesse caso é de $40 \mathrm{~mm}$ de $\mathrm{Hg}$, é chamada de pressão pulso. Sob condições normais, a pressão sistólica nas artérias sistêmicas é a mesma que pressão sistólica no ventrículo esquerdo, uma vez que a válvula aórtica está aberta durante a sístole. Já a pressão diastólica no ventrículo esquerdo é muito menor do que nas artérias. Isso é possível porque a válvula aórtica está fechada durante a diástole.

\section{Teoria de Controle}

Um problema típico da teoria do controle ótimo tem a seguinte forma geral: uma função objetivo que deve ser maximizada; as equações que restringem a função objetivo que podem ser equações diferenciais (que regem os movimentos do sistema, por isso mesmo determinam onde encontra-se o sistema, em que estado ele está. O grupo de variáveis dinâmicas que compõem essas equações é chamado de variáveis de estado) ou equações de igualdade (que impõe algum conceito conhecido do problema ou algum balanço que deve ser atendido pelo sistema); e as condições de transversalidade (condições de contorno ou seja condições iniciais e terminais). Além disso, devem ser especificadas as variáveis de controle com o objetivo de evidenciar quais as variáveis que serão controladas pelo agente através de normas ou políticas.

A função objetivo deve contabilizar os retornos obtidos pelo agente em questão, como o próprio nome sugere, deve refletir o principal objetivo do agente. As restrições sinalizam o conjunto viável pelo qual poderão passar os valores da função objetivo; ela delimita o espaço de ações do agente. As condições de transversalidade fornecem 
informações de onde está se partindo e onde e como deseja-se chegar; essas condições podem ser ou não restritivas aos objetivos do agente.

\section{Modelo Dinâmico}

Nosso objetivo agora é construir um modelo que dinâmico. Para isso vamos considerar inicialmente um vaso com complacência cujo fluxo numa extremidade é $Q_{1}(t)$ e na outra extremidade é $Q_{2}(t)$. Se $V(t)$ é o volume do vaso no instante $t$, podemos considerar a seguinte equação diferencial ordinária:

$$
\frac{d V(t)}{d t}=Q_{1}(t)-Q_{2}(t)
$$

Assumimos também que a equação de complacência é $V(t)=C P(t)$, onde $C$ é a constante de complacência do vaso. Podemos diferenciar a última equação e obter que $\frac{d V(t)}{d t}=C \frac{d P(t)}{d t}$. Assim, temos que $C \frac{d P(t)}{d t}=Q_{1}-Q_{2}$. No nosso caso:

$P=P_{s a}=$ pressão arterial sistêmica,

$C=C_{s a}=$ constante de complacência sistêmica,

$Q_{1}=Q_{L}=$ fluxo sangüíneo do lado esquerdo do coração,

$Q_{2}=Q_{S}=$ fluxo sangüíneo nos tecidos sistêmicos.

Assumimos ainda que $Q_{S}=\frac{P_{s a}-P_{s v}}{R_{S}}$, onde $R_{S}$ é a constante de resistência sistêmica. Como $P_{s v} \ll P_{s a}$, utilizaremos a seguinte equação para o fluxo sistêmico: $Q_{S}=\frac{P_{s a}}{R_{S}}$. Além disso, durante a diástole, $Q_{L}=0$, pois a válvula aórtica está fechada. Ficamos, assim, com a seguinte EDO:

$$
C_{s a} \frac{d P_{s a}(t)}{d t}=-\frac{P_{s a}(t)}{R_{S}}(*)
$$


Em linguagem de controle, temos que $P_{s a}$ é a variável de estado e que $R_{S}$ e $C_{s a}$ são as variáveis de controle.

Uma solução para esta EDO é $P_{s a}(t)=P_{s a}(0) e^{\left(\frac{-t}{R_{s} C_{s a}}\right)}(* *)$. A constante $P_{s a}(0)$ está por ser determinada e para isso vamos considerar a sístole ventricular.

Faremos a hipótese simplificadora de que todo o volume bombeado $\Delta V_{0}$ é ejetado do coração instantaneamente. Nesse caso, podemos usar a equação (*) para a sístole. Como $V(t)=C P(t)$, temos que $\Delta V_{0}=C_{s a} \Delta P_{s a}(\bullet)$, onde $\Delta P_{s a}$ é a pressão pulso.

Suponha agora que a batida cardíaca é um fenômeno periódico, isto é, que cada diástole dura um período $\mathrm{T}$. Como cada batida cardíaca tem período $\mathrm{T}$, a frequiência cardíaca é $\frac{1}{T}$. Assim, a pressão arterial diastólica é $P_{s a}(T)$, a pressão arterial sistólica é $P_{s a}(0)$ e o salto na pressão causado pela ejeção cardíaca é dado por $\Delta P_{s a}=P_{s a}(0)-P_{s a}(T)(\bullet \bullet)$.

Se fizermos $t=T$ em $(* *)$ e substituirmos $(\bullet \bullet)$ em $(\bullet)$, obtemos as seguintes equações:

$P_{s a}(T)=\Theta P_{s a}(0)$, onde $\Theta=e^{\frac{-T}{R_{s} C_{s a}}}$

$\frac{\Delta V_{0}}{C_{s a}}=P_{s a}(0)-P_{s a}(T)$

Como $0<\Theta<1$, podemos, manipulando as equações anteriores, obter:

$P_{s a}(0)=\frac{\Delta V_{0}}{C_{s a}(1-\Theta)} \quad($ sístole $), \mathrm{e}$

$P_{s a}(T)=\frac{\Delta V_{0} \Theta}{C_{s a}(1-\Theta)} \quad$ (diástole).

Essas são as equações para as pressões sistólicas e diastólicas em termos de volume bombeado, complacência arterial, resistência sistêmica e frequiência cardíaca. 
Para obter uma expressão para a pressão pulso, basta subtrair as duas equações anteriores.

Uma observação interessante diz respeito à pressão arterial média. Uma definição para a média de uma função periódica de período $T f(t)$ é $\langle f\rangle=\frac{1}{T} \int_{0}^{T} f(t) d t$, onde $T$ é o período de $f(t)$. Com esta definição, a pressão arterial média é $\left\langle P_{s a}\right\rangle=\frac{1}{T} \int_{0}^{T} P_{s a}(t) d t$. Daí:

$$
\begin{aligned}
& \left\langle P_{s a}\right\rangle=\frac{1}{T} \int_{0}^{T} P_{s a}(0) e^{\frac{-t}{R_{s} C_{s a}}} d t=\frac{\Delta V_{0}}{T C_{s a}(1-\Theta)} \int_{0}^{T} e^{\frac{-t}{R_{s} C_{s a}}} d t=\left.\frac{\Delta V_{0}}{T C_{s a}(1-\Theta)}\left(-R_{S} C_{s a} e^{\frac{-t}{R_{s} C_{s a}}}\right)\right|_{0} ^{T}= \\
& =\frac{-\Delta V_{0}}{T C_{s a}(1-\Theta)}\left(R_{S} C_{s a} e^{\frac{-T}{R_{s} C_{s a}}}-R_{S} C_{s a}\right)=\frac{-\Delta V_{0}}{T C_{s a}(1-\Theta)} R_{S} C_{s a}\left(e^{\frac{-T}{R_{s} C_{s a}}}-1\right)=\frac{\Delta V_{0} R_{S}}{T} .
\end{aligned}
$$

Como $\frac{\Delta V_{0}}{T}=Q$, temos que $\left\langle P_{s a}\right\rangle=Q R_{S}$, que é a equação da pressão arterial sistêmica no caso modelo estático.

Até aqui tratamos do caso em que as diástoles ocorrem com um intervalo de tempo fixo $(T)$. Isso não é exatamente o que ocorre na circulação, em que a freqüência cardíaca e o volume bombeado respondem à frequiência respiratória e às fases da respiração (inspiração e expiração). A hipótese de periodicidade fixa é ainda menos apropriada para indivíduos que apresentem ritmo anormal da freqüência cardíaca, isto é, sucessivas batidas cardíacas apresentam durações e volumes bombeados distintos uma da outra.

Se o volume bombeado e a duração variam de batida para batida, vamos utilizar um índice $(j=1,2,3, \ldots)$ para denotar cada uma das batidas cardíacas. Sejam $t_{j}$ o instante em que ocorre a $j$-ésima batida e $\Delta V_{j}$ o volume bombeado correspondente. Como $P_{s a}(t)$ salta nos instantes $t_{j}$, vamos distinguir pressão arterial imediatamente antes e depois dos instantes $t_{j}$. Assim, sejam

$P_{s a}\left(t_{j}^{-}\right)=$pressão arterial imediatamente antes da diástole, e 
$P_{s a}\left(t_{j}^{+}\right)=$pressão arterial imediatamente após da sístole.

Entre a diátole e a sístole temos, como antes, a EDO:

$C_{s a} \frac{d P_{s a}(t)}{d t}=-\frac{P_{s a}(t)}{R_{S}}$

Agora é mais conveniente escrevermos as soluções na forma: $P_{s a}(t)=P_{s a}\left(t_{j}^{+}\right) e^{\frac{-\left(t-t_{j}\right)}{R_{s} C_{s a}}}$, para $t_{j}<t<t_{j+1}$. Se fizermos $t=t_{j+1}^{-}$temos que $P_{s a}\left(t_{j+1}^{-}\right)=P_{s a}\left(t_{j}^{+}\right) \Theta_{j}$, onde $\Theta_{j}=e^{\frac{-T_{j}}{R_{s} C_{s a}}}$ e $T_{j}=t_{j+1}-t_{j}$. Esta última equação nos fornece a pressão diastólica imediatamente antes da batida $j+1$ em termos da pressão sistólica após a batida $j$.

A equação para o salto na pressão arterial na batida $j$ fica da forma:

$P_{s a}\left(t_{j}^{+}\right)=P_{s a}\left(t_{j}^{-}\right)+\frac{\Delta V_{j}}{C_{s a}}$

Suponha que sejam dados qualquer seqüência de instantes $t_{j}$, volumes bombeados $\Delta V_{j}$ e os parâmetros constantes $C_{s a}$ e $R_{S}$. Se tivermos também a pressão diastólica imediatamente antes da primeira batida, pode-se usar $P_{s a}\left(t_{j}^{+}\right)=P_{s a}\left(t_{j}^{-}\right)+\frac{\Delta V_{j}}{C_{s a}}$ para encontrar a pressão sistólica imediatamente após aquela batida. Podemos, então, usar $P_{s a}\left(t_{j+1}^{-}\right)=P_{s a}\left(t_{j}^{+}\right) \Theta_{j}$ para encontrar a pressão diastólica após a próxima batida cardíaca. Repetindo esse processo, podemos predizer a seqüência inteira de pressões diatólicas e sistólicas por mais irregulares que elas sejam.

O modelo dinâmico apresentado encontra uma maneira elegante de tratar o problema da descontinuidade da pressão arterial sangüínea, porém o que se tem é uma seqüência de EDOs: uma para cada intervalo entre a sístole e a diástole. Isso, porém, faz com que em cada intervalo tenhamos processos independentes, isto é, o que ocorre no final de um processo (entendido aqui como o que ocorre entre uma sístole e uma diástole) não acarreta nenhuma conseqüência no início do processo subseqüente. Além 
disso, os saltos esperados no tratamento da PAS não estão plenamente tratados no modelo com EDOs. Esses fatos serão absorvidos em um modelo de PAS com EDIs. 


\section{2 - Equações Diferenciais com Impulso (EDIs)}

\subsection{Definição e Exemplos}

Seja $M \subseteq \Re^{n}$, onde $\Re$ representa o conjuntos dos números reais, um espaço de fase de um determinado processo evolutivo, isto é, o conjunto de todos os possíveis estados do processo. Denotaremos por $Z$ o conjunto dos números inteiros e por $N$ o conjunto dos números naturais. Seja $x(t)$ o ponto que descreve o estado do processo no instante $t$. Note que um número finito de parâmetros, $n$, descreve o estado em um determinado instante. Com essas hipóteses, um ponto $x(t)$ pode ser interpretado, para um valor fixo de $t$, como um vetor do espaço euclidiano $\Re^{n}$. O produto cartesiano do espaço de fase $M$ e da reta real $\mathfrak{R}, M \times \Re$, será chamado de espaço de fase estendido do processo evolutivo em questão. A evolução do processo é descrita por:

a. Um sistema de equações diferenciais

$$
\frac{d x}{d t}=f(t, x)(1), x \in M, t \in \Re \text {. }
$$

b. Um determinado conjunto $\mathrm{T}$ dado no espaço de fase estendido.

c. Um operador $\mathrm{A}$ definido por $\mathrm{A}: \mathrm{T} \rightarrow \mathrm{T}^{*}=\mathrm{A} \mathrm{T}$ no espaço de fase estendido.

O processo ocorre da seguinte maneira: o ponto $P_{t}=(t, x(t))$ move-se ao longo da curva $\{(t, x(t))\}$ definida pela solução do sistema (1), $x(t)=x\left(t, t_{0}, x_{0}\right)$, tendo iniciado sua trajetória em $\left(t_{0}, x_{0}\right)$. Ele se move nessa curva até o momento $t=t_{1}>t_{0}$, no qual o ponto $(t, x(t))$ encontra o conjunto $\mathrm{T}_{t}$. No momento $t=t_{1}$, o ponto $P_{t}$ é instantaneamente transferido pelo operador $\mathrm{A}$, do ponto $P_{t_{1}}=\left(\mathrm{T}_{1}, x\left(t_{1}\right)\right)$ para o ponto $P_{t_{1}}^{+}=\mathrm{A}_{t_{1}} P_{t_{1}}=\left(t_{1}, x^{+}\left(t_{1}\right)\right) \in \mathrm{T}_{t_{1}}^{*}$ e então continua a se mover ao longo da curva $\{(t, x(t))\}$ descrita pela solução do sistema (1), $x(t)=x\left(t, t_{1}, x^{+}\left(t_{1}\right)\right)$. Ele se move ao longo dessa curva até o momento $t_{2}>t_{1}$, no qual o ponto $P_{t}$ encontra novamente o conjunto $\mathrm{T}_{t}$. Nesse instante, o operador $\mathrm{A}_{t}$ transfere instantaneamente $P_{t}$ do ponto $P_{t_{2}}=\left(t_{2}, x\left(t_{2}\right)\right)$ 
para $P_{t_{2}}^{+}=\mathrm{A}_{t_{2}} P_{t_{2}}=\left(t_{2}, x^{+}\left(t_{2}\right)\right) \in \mathrm{T}_{t_{2}}^{*}$. Ele continua seu movimento ao longo da curva $\{(t, x(t))\}$ determinada pela solução $x(t)=x\left(t, t_{2}, x^{+}\left(t_{2}\right)\right)$ até encontrar-se novamente com o conjunto $\mathrm{T}_{t}$, e assim por diante.

O conjunto de condições “a) - c)", que caracteriza a evolução do processo, é chamado de Sistema de Equações Diferenciais com Impulso. A curva descrita pelo ponto $P$, no espaço de fase estendido, $\{(t, x(t))\}$, será chamada de uma curva integral e a função $x=x(t)$, que nos fornece essa curva, será chamada de uma solução para o sistema (1).

É comum escrever as condições de "a) a c)" de forma mais concisa:

$\frac{d x}{d t}=f(t, x),(t, x) \notin \mathrm{T}$,

$\left.\Delta x\right|_{(t, x) \in \mathrm{T}_{i}}=\mathrm{A} x-x$.

Assim, a solução do sistema (2), $x=\varphi(t)$, é uma função que satisfaz a equação (1) fora do conjunto $T_{t}$ e tem descontinuidades de primeira espécie nos pontos do conjunto $\mathrm{T}$, com os saltos dados por:

$\Delta x=\varphi(t+)-\varphi(t)=\mathrm{A}, \varphi(t)-\varphi(t)$.

Neste trabalho, o sistema (2) (ou o sistema (1)) poderá ter somente um dos três tipos de soluções:

a. As soluções não têm um instante de salto - a curva integral do sistema (1) não intersecta o conjunto $\mathrm{T}$ ou os pontos de intersecção são pontos fixos do operador A ;

b. As soluções têm um número finito de instantes de saltos - a curva integral intersecta o conjunto $\mathrm{T}$ em um número finito de pontos que não são pontos fixos do operador A ;

c. As soluções têm uma quantidade enumerável de instantes de saltos - a curva integral encontra o conjunto $\mathrm{T}$ em uma quantidade enumerável de pontos que não são pontos fixos do operador $\mathrm{A}$. 
Note que as soluções dos sistemas (1) e (2) são funções com descontinuidades de primeira espécie nos pontos $t_{i}, i \in S$, onde $S$ é um subconjunto dos números naturais, que são os pontos da curva $\{(t, x(t))\}$ que encontram o conjunto $\mathrm{T}_{t}$. Isso elimina desse trabalho o caso em que as soluções têm uma quantidade não-enumerável de instantes de salto. Esse fenômeno é chamado de "beating" das soluções e é tratado em [5].

Dependendo das características dos impulsos, há essencialmente três classes de sistemas:

a. Sistemas com impulsos em instantes determinados, isto é sabemos exatamente em que instantes $t$ o ponto $P_{t}$ saltará. As equações dessa classe podem ser escritas da seguinte forma:

$\frac{d x}{d t}=f(t, x), t \neq \tau_{i}$,

$\left.\Delta x\right|_{t=\tau_{i}}=I_{i}(x)$.

Os instantes de impulso são fixados de antemão e ocorrem nos instantes $t=\tau_{i}$, onde $\left\{\tau_{i}\right\}$ é uma dada seqüência de instantes (no máximo enumerável).

b. Sistemas com impulso em instantes tais que os saltos ocorrem nos instantes em que o ponto $P$, intersecta uma dada superfície no espaço de fase estendido. Se tal superfície é dada, no espaço de fase estendido, pela equação $\Phi(t, x)=0$, então as equações dessa classe podem ser escritas da seguinte forma:

$\frac{d x}{d t}=f(t, x), \Phi(t, x) \neq 0$,

$\left.\Delta x\right|_{\phi(t, x)=0}=\left.I(t, x)\right|_{\phi(t, x)=0}$.

c. Sistemas de equações impulsivas autônomas (essa classe é denominada sistemas dinâmicos descontínuos em [1]), que são aqueles em que a equação diferencial dos sistemas (1) ou (2) não depende explicitamente de $t$, os conjuntos $\mathrm{T}_{t} \mathrm{e}$ $\mathrm{T}_{t}^{*}$ são subconjuntos do espaço de fase e o operador $\mathrm{A}_{t}=\mathrm{A}$ para todo $t \in \mathfrak{R}$. Se fizermos $\mathrm{T}_{t}=\Gamma, \mathrm{T}_{t}^{*}=\Gamma_{0}$ e $\mathrm{A}: \Gamma \rightarrow \Gamma_{0}$, então as equações dessa classe podem ser escritas da seguinte forma: 


$$
\begin{aligned}
& \frac{d x}{d t}=f(x), x \notin \Gamma, \\
& \left.\Delta x\right|_{x \in \Gamma}=\mathrm{A} x-x=I(x) .
\end{aligned}
$$

Vamos a seguir apresentar três exemplos de EDIs.

Exemplo 1 - Considere o conjunto $\mathrm{T}_{t}=\left\{(t, x) \in \mathfrak{R}^{2} / x=\operatorname{atan}(\tan (t))\right\}$, o operador $\mathrm{A}_{t}$ dado pela fórmula $\mathrm{A}_{t}(t, x)=\left(t, x^{2} \operatorname{sigh}(x)\right)$ e o sistema de equações diferenciais dado por $\frac{d x}{d t}=0$. O sistema pode ser reescrito como em (2):

$$
\left\{\begin{array}{c}
\frac{d x}{d t}=0, \quad(t, x) \notin T \\
\left.\Delta x\right|_{(t, x) \in T_{r}}=x^{2} \operatorname{sign}(x)-x
\end{array}\right.
$$

Vamos analisar as possíveis trajetórias desse sistema. Temos os seguintes casos:

(i) A trajetória inicia em $t=0$ no ponto $x_{0},\left|x_{0}\right| \geq \frac{\pi}{2}$. Nesse caso a trajetória é estacionária, pois a curva integral de tal movimento, que é a linha constante $x(t)=x_{0}$, não encontra o conjunto $\mathrm{T}_{\mathrm{t}}$ em nenhum $t \geq 0$. Esse caso pode ser visto na figura abaixo. A solução é a curva vermelha: 


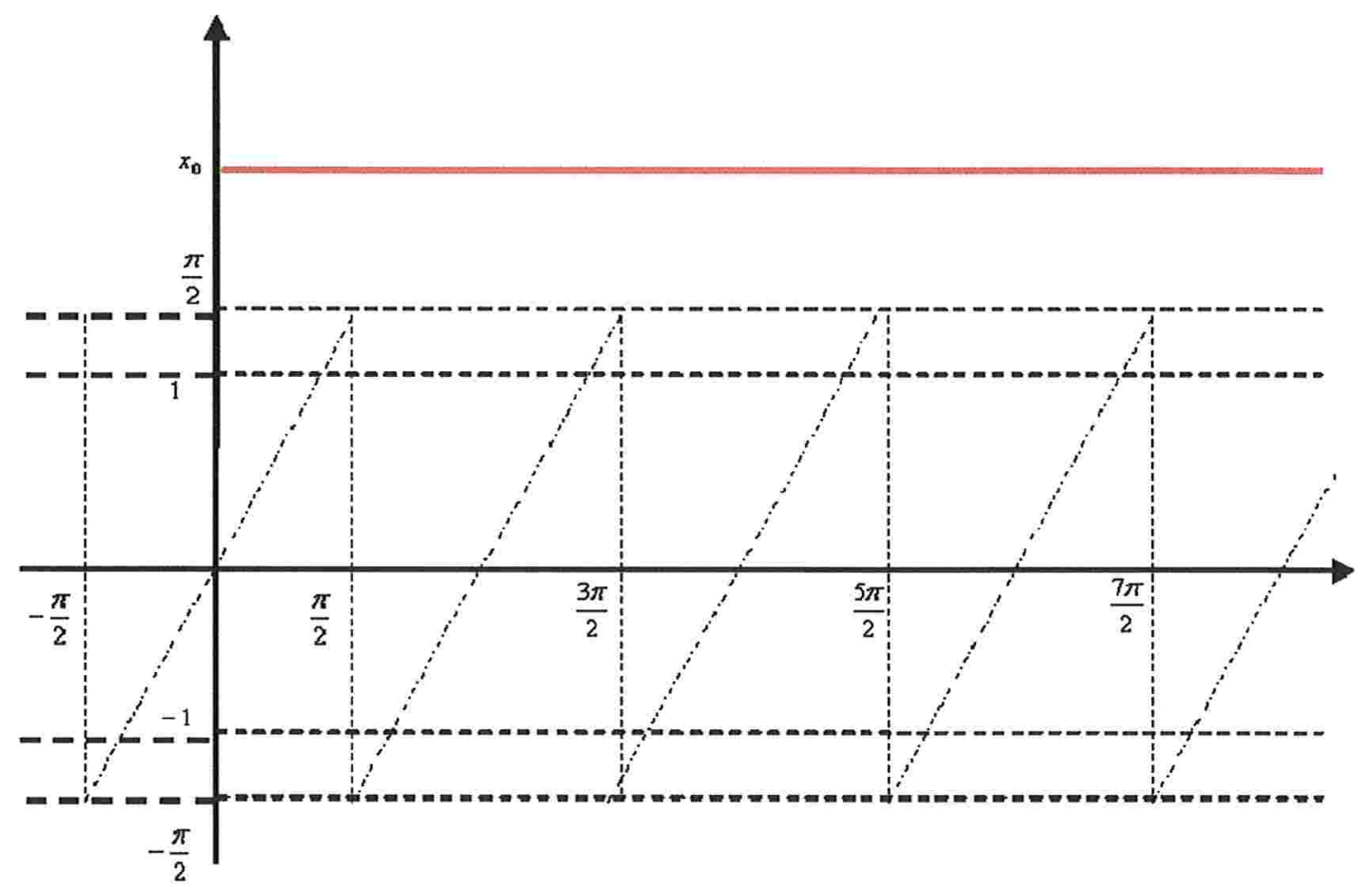

(ii) A trajetória inicia em $t=0$ no ponto $x_{0}, 1 \leq\left|x_{0}\right| \leq \frac{\pi}{2}$. Nesse caso a trajetória tem um número finito de impulsos, pois a curva integral de tal movimento encontra o conjunto $\mathrm{T}_{t}$ em um número finito de vezes. Por exemplo, se a trajetória inicia em $\mathrm{t}=0$ no ponto $x=\sqrt[8]{2}$, então a trajetória assume quatro valores: $x=\sqrt[8]{2}, \sqrt[4]{2}, \sqrt{2}, 2$. Depois de assumir o valor $x=2$ a trajetória não encontra mais o conjunto $\mathrm{T}_{t}$ e não há mais, portanto, saltos na trajetória. Esse caso pode ser visto na figura abaixo. A solução é a curva vermelha: 


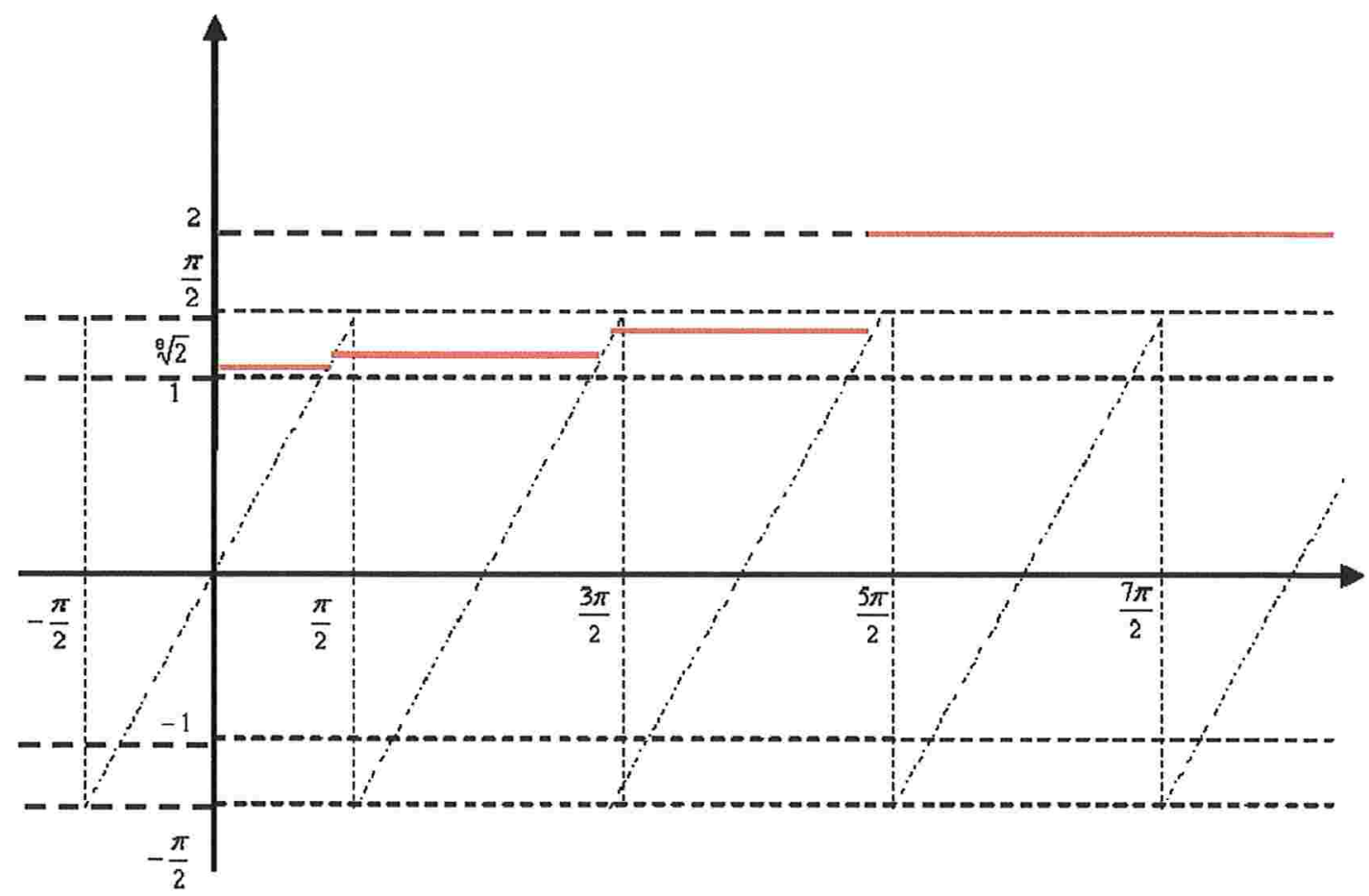

(iii) A trajetória inicia em $t=0$ no ponto $x_{0},\left|x_{0}\right| \leq 1$. Nesse caso a trajetória tem uma quantidade enumerável de saltos. Por exemplo, se a trajetória inicia no ponto $x=\frac{1}{2}$ no instante $t=0$, então a trajetória é o conjunto dos pontos $x=\frac{1}{2^{n}}, n=0,1,2 \ldots$. Esse caso pode ser visto na figura abaixo. A solução é a curva vermelha: 


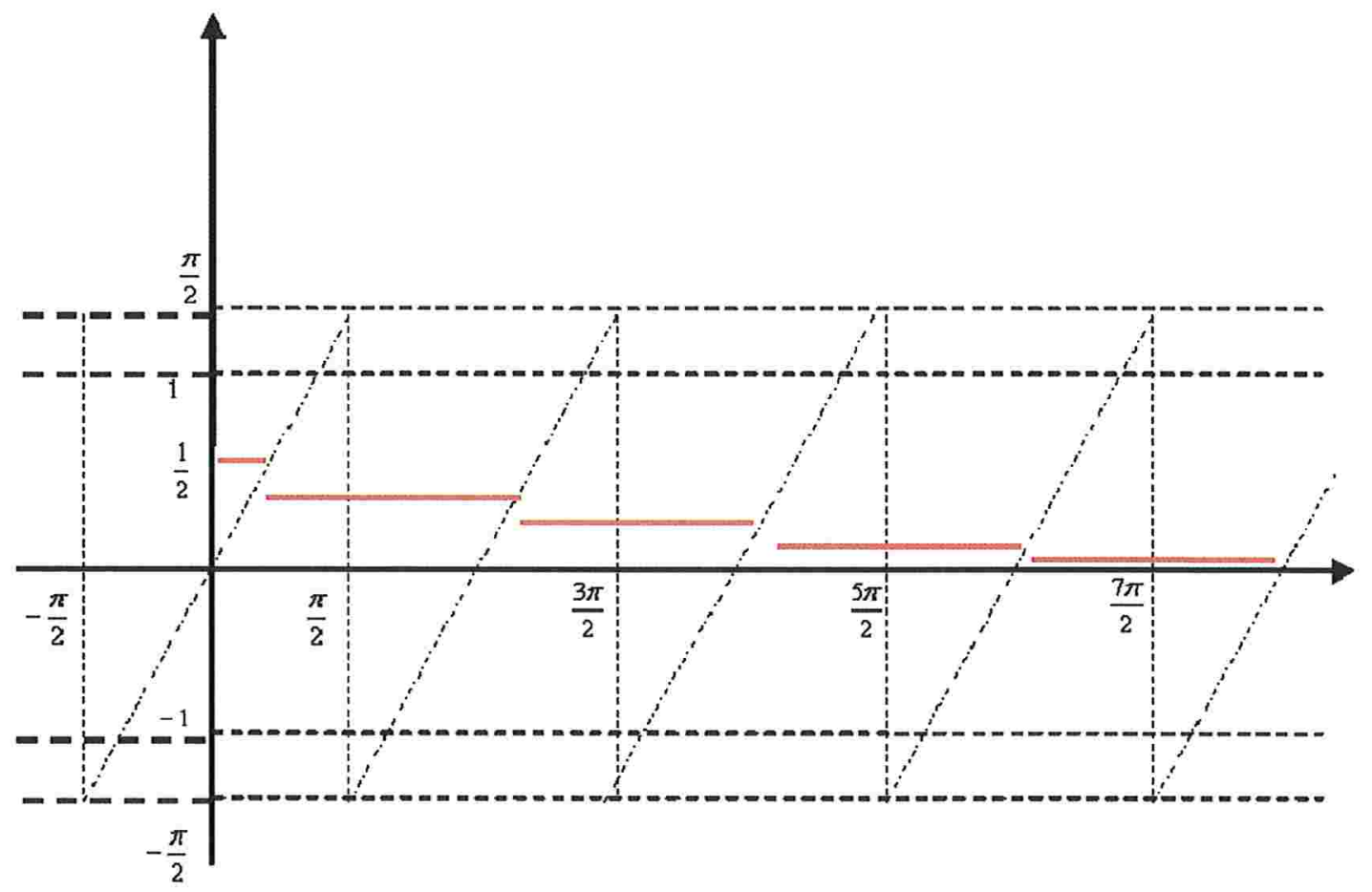

(iv) As trajetórias que em $t=0$ iniciam em $x=0$ ou $x= \pm 1$ intersectam 0 conjunto $T$, numa quantidade enumerável de vezes, mas não há saltos, pois essas trajetórias são estacionárias. $\mathrm{O}$ caso $x=1$ pode ser visto na figura abaixo. A solução é a curva vermelha: 


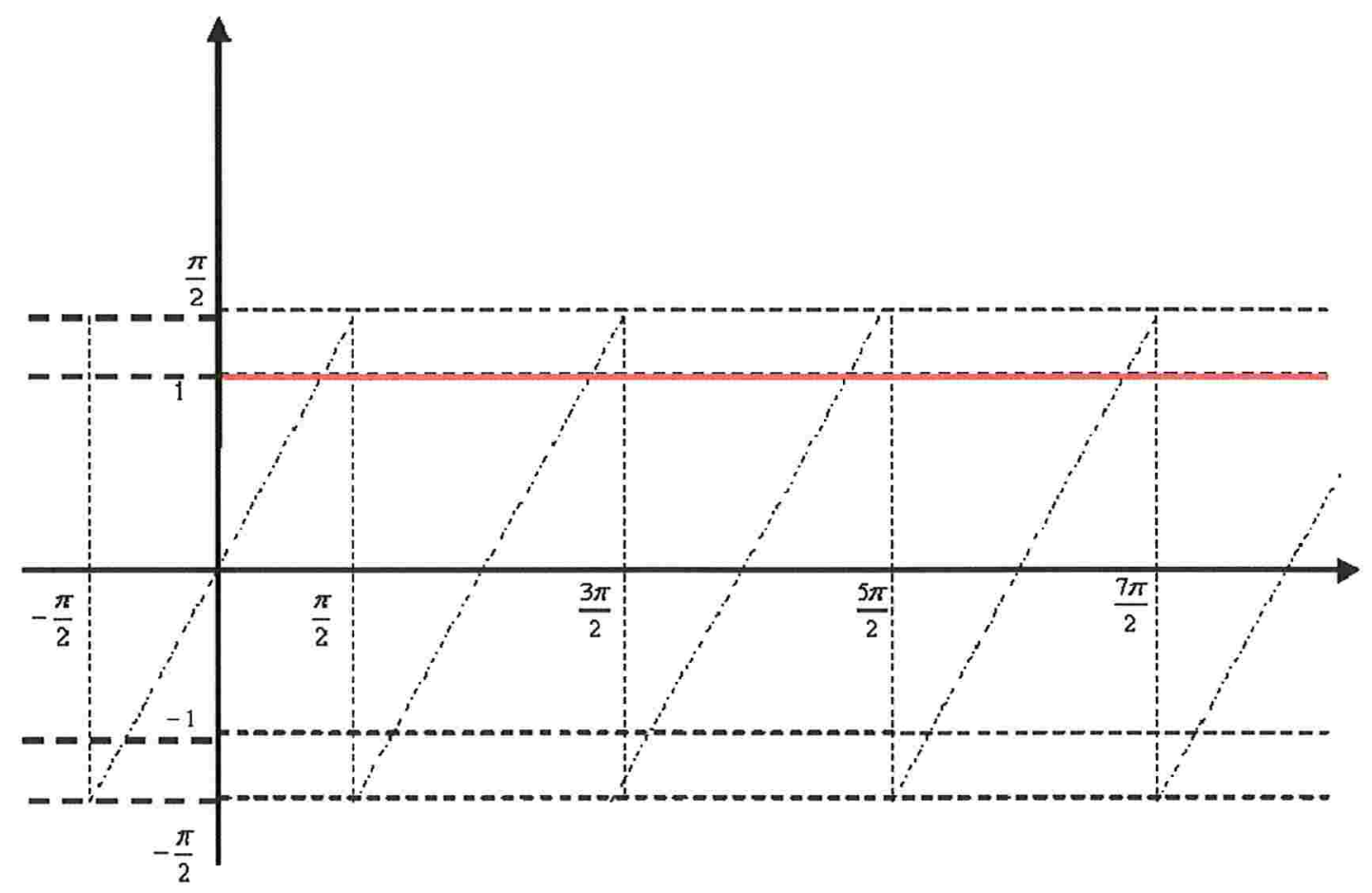

Exemplo 2 - Considere o ponto $(x, y)$ que se move no plano $\Re^{2}$ e seu movimento é descrito por:

$\frac{d x}{d t}=0, \quad \frac{d y}{d t}=-1$

Sejam $\mathrm{T}_{t}$ o eixo $x$ (a reta $y=0$ ) e $\mathrm{T}_{0}$ o semi-círculo $x^{2}+(y-2)^{2}=1, y<2$. O operador $A_{t}: T_{t} \rightarrow T_{0}$ age da seguinte maneira: o ponto $(x, 0)$ é levado para o ponto da intersecção entre o semi-círculo e a reta que passa pelos pontos $(x, 0)$ e $(0,2)$. Vamos denotar por $O$ a origem $(0,0)$ e por $B$ o ponto $(0,2)$.

Todas as soluções desse sistema que iniciam em $\left(x_{0}, y_{0}\right) \in \mathfrak{R}^{2}, y_{0}<0$, não apresentam saltos. Isso pode ser visto na figura a seguir. A solução é a curva vermelha. 




As soluções que iniciam em $\left(0, y_{0}\right) \in \Re^{2}, 0 \leq y_{0} \leq 1$ são periódicas. Isso pode ser visto na figura a seguir. A solução é a curva vermelha.

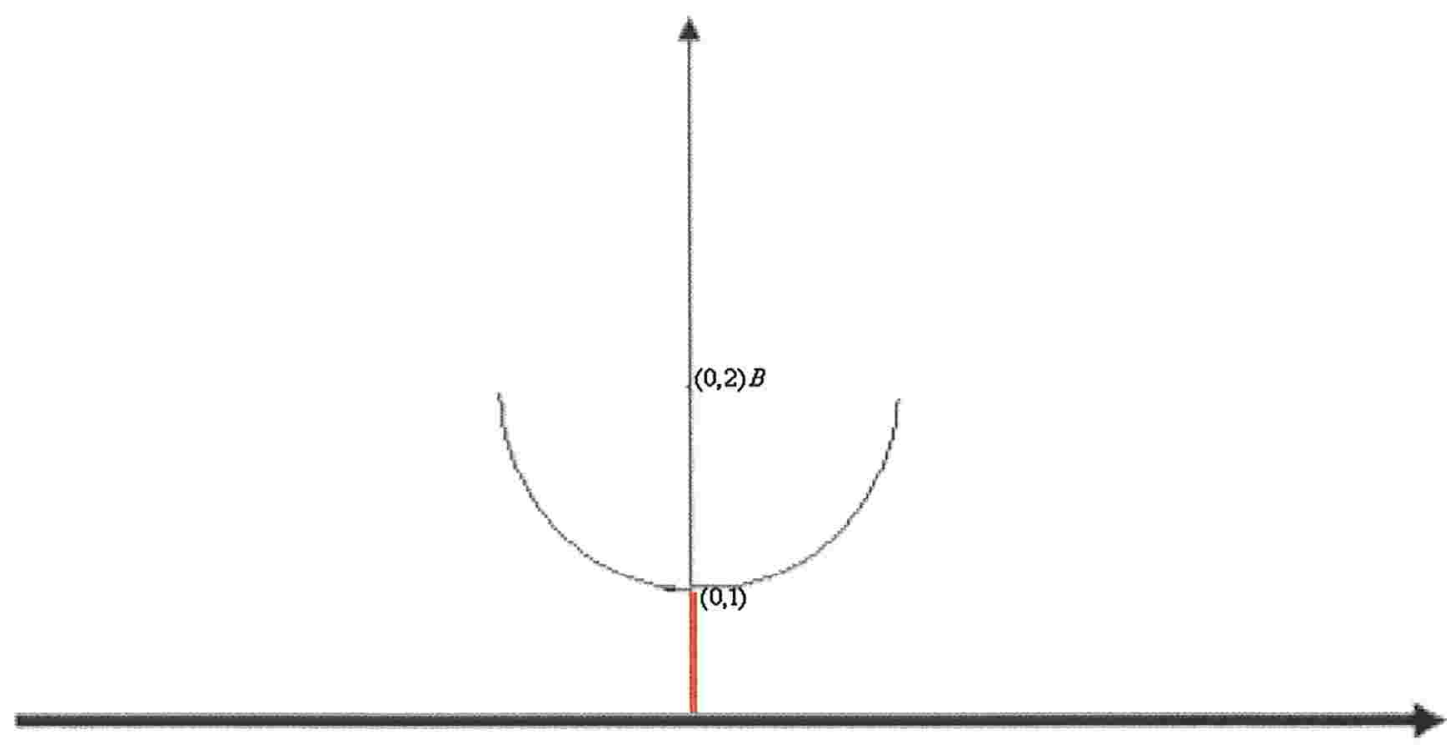


Já as soluções $\left(0, y_{0}\right) \in \Re^{2}, y_{0}>1$ tornam-se periódica após o primeiro salto. Isso pode ser visto na figura a seguir. A solução é a curva vermelha.

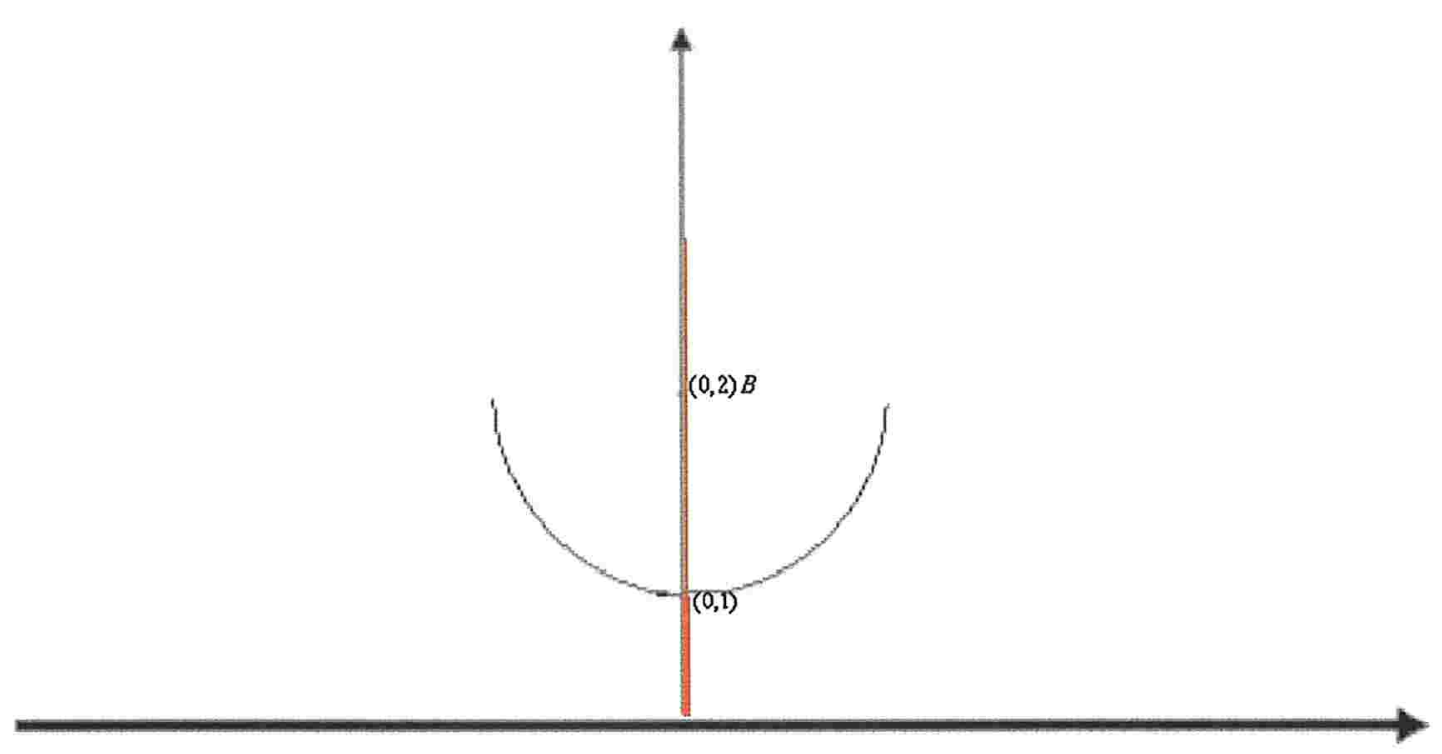

Todas as outras soluções apresentam uma quantidade enumerável de saltos e quando $t \rightarrow \infty$, aproximam-se da solução periódica, que é dada pelo segmento $O B$ que está no eixo y. Assim, o segmento $O B$ atrai as trajetórias de todos as soluções que iniciam nos pontos $(x, y), y \geq 0$. Isso pode ser visto na figura a seguir. A solução é a curva vermelha.

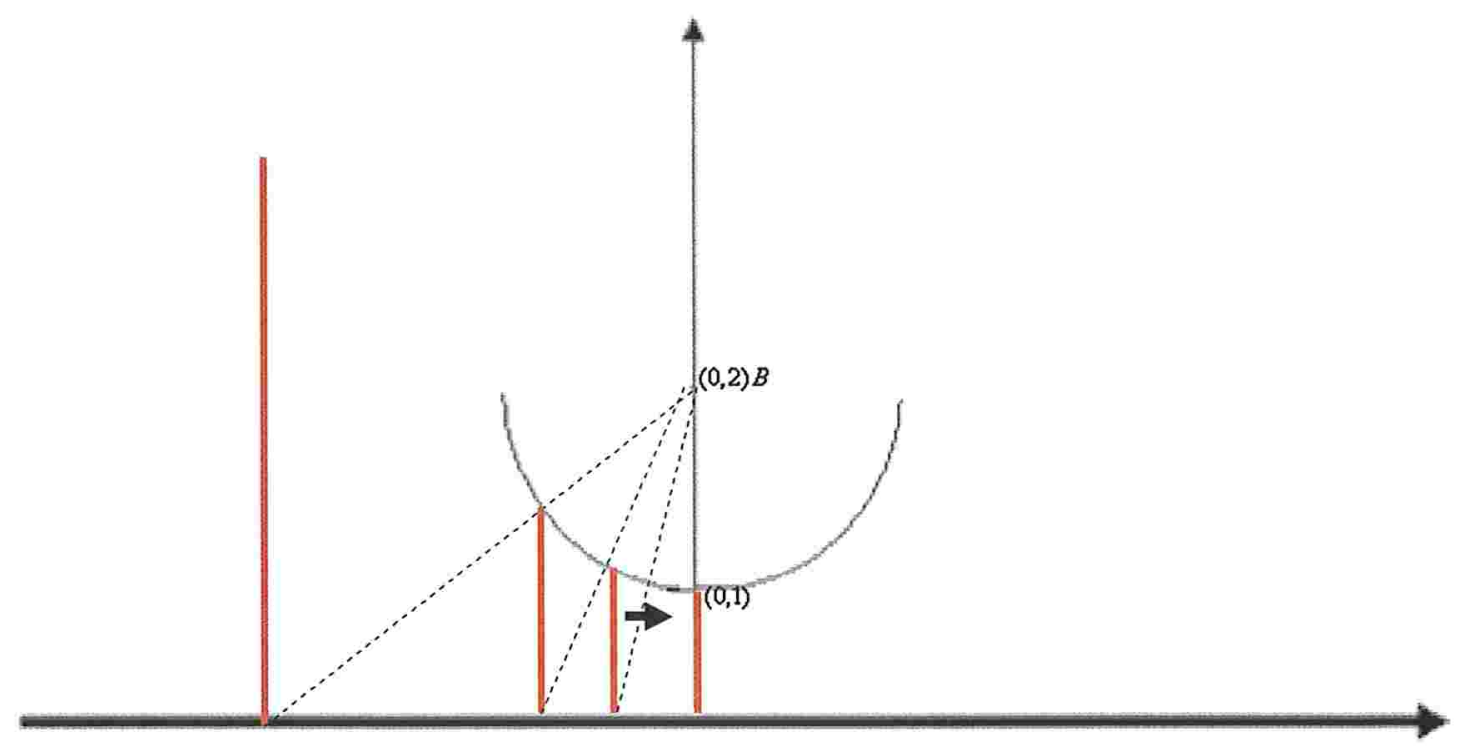


Exemplo 3 - Considere duas circunferências com raios $r$ e $R, R>r$, e com mesmo centro como na figura a seguir:

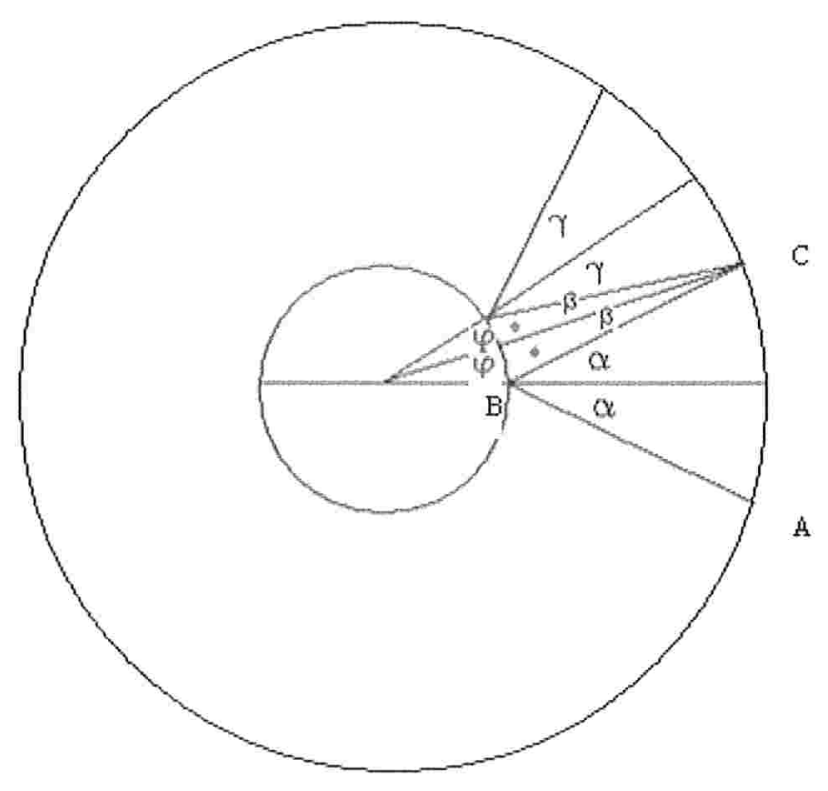

O sistema em questão é descrito da seguinte maneira: A solução parte de um ponto A (como na figura) e sua trajetória é uma reta cujo ângulo com a componente normal $\mathrm{N}$ da circunferência de raio $\mathrm{r}$ é $\alpha$. Ao intersectar com a circunferência de raio $\mathrm{r}$ no ponto $\mathrm{B}$, a trajetória é imediatamente transferida pelo operador $A_{t}$ para o ponto $C$, que corresponde à reflexão do ponto $\mathrm{A}$ no que diz respeito à componente normal $\mathrm{N}$. A seguir a trajetória parte do ponto $\mathrm{C}$, novamente como uma reta cujo ângulo com a componente normal N' da circunferência de raio r é $\gamma$, e assim por diante. No triângulo com bola vermelha, note que $\varphi+\beta+\pi-\alpha=\pi$, donde $\varphi+\beta=\alpha$. Agora note que no triângulo com bola verde $\varphi+\beta+\pi-\gamma=\pi$, donde $\varphi+\beta=\gamma$. Logo $\alpha=\gamma$. Desta forma somente haveria ciclo se $k 2 \alpha=2 \pi$, isto é, se $\pi$ for um múltiplo inteiro de $\alpha$. Caso contrário, as trajetórias formam um conjunto denso na área compreendida entre as duas circunferências. 


\subsection{Solução de Sistemas Lineares de EDIs}

Nesta seção vamos estabelecer algumas propriedades dos sistemas de equações diferenciais impulsivos lineares homogêneos $(\mathrm{H})$ escritos da seguinte forma:

$$
\frac{d x}{d t}=A(t) x, \quad t \neq \tau_{i},\left.\quad \Delta x\right|_{t=\tau_{i}}=B_{i} x \quad(3) .
$$

Trataremos também o caso não-homogêneo $(\mathrm{NH})$, que será escrito da seguinte forma:

$$
\frac{d x}{d t}=A(t) x+f(t), \quad t \neq \tau_{i},\left.\quad \Delta x\right|_{t=\tau_{i}}=B_{i} x+a_{i}
$$

Nos sistemas (3) e (4), $A(t)$ é uma matriz contínua $n \times n$ definida em um intervalo aberto $I, B_{i}$ são matrizes constantes $n \times n, \tau_{i} \in I$ são instantes fixados indexados por um subconjunto dos números inteiros tais que $\tau_{i}<\tau_{i+1}, f(t)$ é uma função contínua (ou contínua por partes) no intervalo $I$ e $a_{i}$ são vetores constantes.

Teorema 1 - Seja o intervalo $\left[t_{0}, t_{0}+h\right] \subset I$ contendo um número fínito de pontos $\tau_{i}$. Então, para qualquer $x_{0} \in \mathfrak{R}^{n}$, existe uma solução do sistema (3) $x\left(t, x_{0}\right), x\left(t_{0}, x_{0}\right)=x_{0}$ para todo $t \in\left[t_{0}, t_{0}+h\right]$. Além disso, se, para todo $i$ tal que $\tau_{i} \in\left[t_{0}, t_{0}+h\right]$, as matrizes $I d+B_{i}$ forem não-singulares, onde $I d$ representa a matriz identidade, então $x\left(t, x_{0}\right) \neq x\left(t, y_{0}\right)$ para todo $t \in\left[t_{0}, t_{0}+h\right]$ se $x_{0} \neq y_{0}$.

Demonstração:

Seja $t_{0}<\tau_{j}<\tau_{j+1}<\ldots<\tau_{j+k} \leq t_{0}+h$. Pelo teorema de Picard, existe uma solução $x=\varphi_{j}(t), \varphi_{j}\left(t_{0}\right)=x_{0}$ do sistema $\frac{d x}{d t}=A(t) x$ no intervalo $\left[t_{0}, \tau_{j}\right]$ para qualquer 
$x_{0} \in \Re^{n}$ e tal solução é única. Se fizermos $t=\tau_{j}$ em (3) temos que $x\left(\tau_{j}+0, x_{0}\right)=\left(I d+B_{j}\right) x\left(\tau_{j}, x_{0}\right)=x_{j}^{+}$, onde $x\left(\tau_{j}+0, x_{0}\right)=\lim _{l \rightarrow 0+} x\left(\tau_{j}+l, x_{0}\right)$.

Novamente aplicando o Teorema de Picard, vemos que existe em $\left[\tau_{j}, \tau_{j+1}\right]$ uma única solução $x=\varphi_{j+1}(t), \varphi_{j+1}\left(\tau_{j}\right)=x_{j}^{+}$do sistema $\frac{d x}{d t}=A(t) x$. Assim, a solução de (3) pode ser estendida até $t=\tau_{j+1}$ fazendo-se $x\left(t, x_{0}\right)=\varphi_{j+1}(t)$ para $\tau_{j}<t<\tau_{j+1}$, e $x\left(\tau_{j+2}(t)+0, x_{0}\right)=\left(I d+B_{j+1}\right) x\left(\tau_{j}, x_{0}\right)$ para $t=\tau_{j+1}+0$.

Denotando por $\varphi_{j+2}(t) \quad$ a solução de $\quad \frac{d x}{d t}=A(t) x$ tal que $\varphi_{j+2}\left(\tau_{j+1}\right)=\left(I d+B_{j+1}\right) x\left(\tau_{j+1}, x_{0}\right)=x_{j+1}^{+}$, podemos estender a solução $x\left(t, x_{0}\right)$ até o instante $\tau_{j+2}$ fazendo $x\left(t, x_{0}\right)=\varphi_{j+2}(t)$ para $\tau_{j+1}<t<\tau_{j+2}$ e assim por diante. Como o intervalo $\left[t_{0}, t_{0}+h\right]$ contém um número finito de pontos $\tau_{j}$, utilizando o procedimento aqui descrito conseguiremos uma solução $x\left(t, x_{0}\right)$ para o intervalo todo.

Até aqui assumimos que $t_{0}<\tau_{j}$. Se $t_{0}=\tau_{j}$, então usamos a mesma construção feita para $t_{0}<\tau_{j}$. A diferença é que fazemos $x\left(t_{0}+0, x_{0}\right)=\left(I d+B_{j}\right) x_{0}$ e construímos a função $\varphi_{j+1}(t)$, solução de $\frac{d x}{d t}=A(t) x$, que satisfaz a condição inicial $\varphi_{j+1}\left(\tau_{j}\right)=\left(I d+B_{j}\right) x_{0}=x_{j}^{+}$.

Para provar a segunda afirmação do teorema, observamos que se $x\left(\tau_{i}+0, x_{0}\right) \neq x\left(\tau_{i}+0, y_{0}\right)$, então pelo Teorema de Picard temos que $x\left(t, x_{0}\right) \neq x\left(t, y_{0}\right)$ para todo $\tau_{i}<t<\tau_{i+1}, i=j, j+1, \ldots, j+k$.

Como $\quad x\left(\tau_{i}+0, x_{0}\right)-x\left(\tau_{i}+0, y_{0}\right)=\left(I d+B_{i}\right)\left(x\left(\tau_{i}, x_{0}\right)-x\left(\tau_{i}, y_{0}\right)\right), \quad$ segue $\quad$ de $x\left(\tau_{i}, x_{0}\right) \neq x\left(\tau_{i}, y_{0}\right)$ que $x\left(\tau_{i}+0, x_{0}\right) \neq x\left(\tau_{i}+0, y_{0}\right)$ se a matriz $I d+B_{i}$ é não-singular. Isso implica que $x\left(t, x_{0}\right) \neq x\left(t, y_{0}\right)$ para todo $t \in\left[t_{0}, t_{0}+h\right]$ se $x_{0} \neq y_{0} \quad \mathrm{e}$ $\operatorname{det}\left(I d+B_{i}\right) \neq 0$ para $i=j, j+1, \ldots, j+k$.

O teorema 1 está para as EDIs assim como o Teorema de Picard está para as EDOs. Temos a existência e unicidade de solução para o problema de Cauchy. Na segunda afirmação do teorema 1, foi feita uma aplicação do teorema de Picard para 
EDOs nos intervalos determinados pelo momento imediatamente posterior a um salto e imediatamente anterior ao salto seguinte.

No que segue, estudaremos somente os sistemas que satisfazem as seguintes condições:

1) Qualquer intervalo compacto $[a, b]$ contém somente um número finito de pontos $\tau_{i}$.

2) Para cada $i$ tal que $\tau_{i} \in I$, as matrizes $I d+B_{i}$ são não-singulares.

Teorema 2 - Sob as condições 1) e 2) acima, o conjunto $\chi$ de todas as soluções do sistema (3), que estão definidas em um intervalo $[a, b]$ formam um espaço vetorial de dimensão $n$.

Demonstração:

Sejam $\varphi_{1}(t)$ e $\varphi_{2}(t)$ soluções do sistema (3) definidas no intervalo $[a, b], c_{1}$ e $c_{2}$ números reais. Claramente $c_{1} \varphi_{1}(t)+c_{2} \varphi_{2}(t)$ é também solução de (3). Assim, as soluções formam um espaço linear.

Para mostrarmos que a dimensão é $n$, vamos mostrar que o conjunto $\chi$ é isomórfico ao espaço de fase do sistema, isto é, o espaço $\Re^{n}$.

De fato, seja $t \in[a, b]$. Considere a aplicação $g_{1}: \chi \rightarrow \mathfrak{R}^{n}, g_{t} \varphi=\varphi(t)$. Note que a aplicação $g_{1}: \chi \rightarrow \Re^{n}$ é linear, sua imagem é todo o espaço $\Re^{n}$, pois pelo Teorema 1 , para qualquer $x_{0} \in \Re^{n}$, existe uma solução $\varphi(t)=x\left(t, x_{0}\right), \varphi\left(t_{0}\right)=x_{0}$. Além disso, 0 núcleo da aplicação $g_{l}: \chi \rightarrow \Re^{n}$ é somente o 0 , pois pelo Teorema $1, \varphi\left(t_{0}\right)=0$ implica $\varphi(t) \equiv 0$.



Esse resultado é o mesmo que para as EDOs, isto é, suas soluções formam um espaço vetorial.

Uma base do espaço $\chi$ é chamada sistema fundamental de soluções de (3). Esse último teorema tem alguns corolários imediatos: 
1) O sistema (3) tem um sistema fundamental de n soluções $\varphi_{1}(t), \varphi_{2}(t), \ldots, \varphi_{n}(t)$;

2) Toda solução de (3) é combinação linear das soluções do sistema fundamental;

3) Se tivermos $n+1$ soluções de (3), então elas formam um conjunto linearmente dependente.

Denotaremos por $X(t)$ a matriz cujas colunas são soluções do sistema (3) que formam o sistema fundamental. A matriz $X(t)$ será chamada matriz fundamental do sistema (3). É claro que a função $x(t)=X(t) c$, onde $c$ é um vetor constante qualquer, é uma solução de (3). Pela definição da matriz $X(t)$, segue que ela satisfaz a seguinte equação impulsiva matricial:

$$
\frac{d X}{d t}=A(t) X, \quad t \neq \tau_{i},\left.\quad \Delta X\right|_{t=\tau_{i}}=B_{i} X(5) .
$$

A relação entre os sistemas (3) e (4) é estabelecida no seguinte teorema:

Teorema 3 - Se $x=\varphi(t)$ é uma solução do sistema (3) e $x=\psi(t)$ é solução do sistema (4), então a função $x=\varphi(t)+\psi(t)$ é solução de (4). Inversamente, se $x=\varphi_{1}(t)$ e $x=\varphi_{2}(t)$ são soluções de (4), então $x=\varphi_{1}(t)-\varphi_{2}(t)$ é solução de (3).

Demonstração:

Basta substituir as soluções nos sistemas (3) e (4) e verificar que de fato são soluções.

Teorema 4 - Seja $S$ uma matriz não-singular, contínua por partes e diferenciável com relação a $t \in[a, b] \backslash\left\{\tau_{i}\right\}$. Então, se $x=S(t) y$, o sistema NH (4) pode ser escrito como:

$$
\begin{aligned}
& \frac{d y}{d t}=S^{-1}(t)\left[A(t) S(t)-\frac{d S}{d t}\right] y+S^{-1}(t) f(t), t \neq \tau_{i}, \\
& \left.\Delta y\right|_{t=\tau_{i}}=\left.S^{-1}\left(\tau_{i}+0\right)\left(-\Delta S+B_{i} S\right) y\right|_{t=\tau_{i}}+S^{-1}\left(\tau_{i}+0\right) a_{i} .
\end{aligned}
$$

Demonstração: 
Basta diferenciar $x=S(t) y$ com relação a $t$ e substituir em (4).

Se, em particular, $S(t)$ é uma matriz fundamental do sistema $\frac{d x}{d t}=P(t) x, t \neq \tau_{i}$, $\left.\Delta x\right|_{t=\tau_{i}}=I_{i} x$, onde as matrizes $I d+I_{i}$ são não-singulares, então o sistema (4) pode, aplicando o teorema 4, ser escrito como:

$$
\begin{aligned}
& \frac{d y}{d t}=S^{-1}(t)[A(t)-P(t)] S(t) y+S^{-1}(t) f(t), t \neq \tau_{i} \\
& \left.\Delta y\right|_{t=\tau_{i}}=\left.S^{-1}\left(\tau_{i}+0\right)\left(B_{i}-I_{i}\right) S y\right|_{t=\tau_{i}}+S^{-1}\left(\tau_{i}+0\right) a_{i} .
\end{aligned}
$$

Observamos que se $A(t)=P(t), B_{i}=I_{i}$ e $S(t)=X(t)$, onde $X(t)$ é a matriz fundamental do sistema (3), a mudança de variável $x=S(t) y$ é chamada variação de parâmetros. Nesse caso, a igualdade acima (e, portanto o sistema (4)) pode ser escrito como:

$$
\frac{d y}{d t}=X^{-1}(t) f(t), t \neq \tau_{i},\left.\Delta y\right|_{t=\tau_{i}}=X^{-1}\left(\tau_{i}+0\right) a_{i} .
$$

Levando-se em conta que $X\left(\tau_{i}+0\right)=\left(I d+B_{i}\right) X\left(\tau_{i}\right)$, a condição de salto de (6) pode ser reescrita como $\left.\Delta y\right|_{t=\tau_{i}}=X^{-1}\left(\tau_{i}\right)\left(I d+B_{i}\right)^{-1} a_{i}$. Usando essa expressão e integrando (5) com relação a $\mathrm{t}$, encontramos para $t \geq t_{0}$ :

$$
y(t)=c+\int_{t_{0}}^{t} X^{-1}(\tau) f(\tau) d \tau+\sum_{t_{11}<\tau_{i}<t} X^{-1}\left(\tau_{i}\right)\left(I d+B_{i}\right)^{-1} a_{i}
$$

onde $c=y\left(t_{0}\right)$ é um vetor constante.

Com isso, temos imediatamente o seguinte corolário:

Corolário 1 - Seja $X(t)$ a matriz fundamental do sistema (3) com matrizes nãosingulares $I d+B_{i}$. Então, toda solução do sistema (4), para $t \geq t_{0}$ é dada pela fórmula: 


$$
x(t)=X(t)\left[c+\int_{t_{0}}^{t} X^{-1}(\tau) f(\tau) d \tau+\sum_{t_{u}<\tau_{i}<t} X^{-1}\left(\tau_{i}\right)\left(I d+B_{i}\right)^{-1} a_{i}\right] .
$$

\subsection{Oscilação, Estabilidade e Periodicidade em Sistemas de EDIs}

Qualquer solução do sistema matricial (5) é uma matriz fundamental do sistema (3). Além disso, toda solução do sistema matricial (5) é dada por $X(t)=X_{0}(t) C$, onde $X_{0}(t)$ é uma solução de (5) e C é uma matriz arbitrária e não-singular qualquer. Esse fato decorre imediatamente dos teoremas 1 e 2. Uma solução de (5) que satisfaz a condição $X\left(t_{0}\right)=I d$ é chamada de matriciante do sistema (3) e é denotada por $X\left(t, t_{0}\right)$.

Denotaremos por $P C$ o espaço das funções contínuas por partes, isto é funções que são descontínuas numa quantidade no máximo enumerável de pontos definida em $\Re$ e com descontinuidades de primeira espécie.

Seja $a_{i}, i \in Z$, uma seqüência em $\Re$. Dizemos que a sequiência $a_{i}$ é $\varepsilon$-quaseperiódica se existe um inteiro $p$, chamado de um $\varepsilon$-quase - período da sequiência $a_{i}$, se $\left|a_{i+p}-a_{i}\right| \leq \varepsilon, i \in Z$. Se $\varepsilon=0, p$ é chamado de período e nesse caso a seqüência $a_{i}$ é uma seqüência periódica.

Um conjunto $S \subseteq \Re$ é chamado de relativamente denso se existe um número $l>0$ tal que $[a, a+l] \cap S \neq \varnothing$ para todo $a \in \Re$. O conjunto $\Re \backslash[0,1]$ não é denso em $\Re$, mas é relativamente denso se tomarmos $l=2$. Outro exemplo de conjunto que não é denso em $\mathfrak{R}$, mas é relativamente denso em $\mathfrak{R}$ é o conjunto dos números naturais $\mathrm{N}$ $\operatorname{com} l=1$. Todo conjunto denso em $\Re$ é relativamente denso com qualquer $l$.

Uma seqüência $a_{i}$ é chamada de quase-periódica se para qualquer $\varepsilon>0$ existe um conjunto relativamente denso $S$ de $\varepsilon$-quase-períodos, isto é, existe um número natural $N=N(\varepsilon)$ tal que, para um número arbitrário $k \in Z$, existe pelo menos um número $p$ no intervalo $[k, k+N]$, para o qual $\left|a_{i+p}-a_{i}\right| \leq \varepsilon, i \in Z$. 
Seja $\Theta_{i}$ uma seqüência de números reais tal que $\Theta_{i}<\Theta_{i+1}$ e $\left|\Theta_{i}\right| \rightarrow \infty$, quando $i \rightarrow \infty$. Vamos denotar $\Theta_{i}^{j}=\Theta_{i+j}-\Theta_{i}$ para todos os inteiros $i, j$.

É fácil verificar que $\Theta_{i+k}^{j}-\Theta_{i}^{j}=\Theta_{i+j}^{k}-\Theta_{i}^{k}$ e que $\Theta_{i}^{j}-\Theta_{i}^{k}=\Theta_{i+k}^{j-k}$.

De fato,

$$
\begin{gathered}
\Theta_{i+k}^{j}-\Theta_{i}^{j}=\left(\Theta_{i+j+k}-\Theta_{i+k}\right)-\left(\Theta_{i+j}-\Theta_{i}\right)= \\
\left(\Theta_{i+j+k}-\Theta_{i+j}\right)-\left(\Theta_{i+k}-\Theta_{i}\right)=\Theta_{i+j}^{k}-\Theta_{i}^{k}
\end{gathered}
$$

Para a outra afirmação,

$$
\begin{aligned}
& \Theta_{i+k}^{j-k}=\Theta_{i+k+j-k}-\Theta_{i+k}= \\
& =\left(\Theta_{i+j}-\Theta_{i}\right)-\left(\Theta_{i+k}-\Theta_{i}\right)=\Theta_{i}^{j}-\Theta_{i}^{k}
\end{aligned}
$$

Uma família de seqüências $\Theta_{i}^{j}, i, j \in Z$ é chamada equipotencialmente quaseperiódica se, para um $\varepsilon>0$ arbitrário, existe um conjunto relativamente denso de $\varepsilon$ quase-períodos que são comuns a todas as seqüências $\left\{\Theta_{i}^{j}\right\}$.

A seguir introduziremos o conceito de função quase-periódica. Observamos que ao restringirmos o domínio de uma função quase-periódica ao conjunto dos inteiros $Z$, obtemos uma seqüência quase-periódica. Assim, a partir das funções que exibiremos como exemplo a seguir, é possível que sejam obtidas seqüências quase-periódicas.

Uma função $T: \Re \rightarrow C$, onde $C$ representa o conjunto dos números complexos, $T(x)=\sum_{k=1}^{n} c_{k} e^{i \lambda_{k} x}$, onde $c_{k}$ são números complexos e $\lambda_{k}$ são números reais, é chamada de polinômio trigonométrico complexo.

Observamos que se $T(x)$ é um polinômio trigonométrico, então seu conjugado, $\overline{T(x)}$, é também um polinômio trigonométrico. De fato, $\overline{T(x)}=\overline{\sum_{k=1}^{n} c_{k} e^{i \lambda_{k} x}}=\sum_{k=1}^{n} \overline{c_{k}} \overline{e^{i \lambda_{k} x}}=\sum_{k=1}^{n} \overline{c_{k}} e^{-i \lambda_{k} x}$.

Observamos também que se $T(x)=\sum_{k=1}^{n} c_{k} e^{i \lambda_{k} x} \quad$ e $\quad S(x)=\sum_{k=1}^{m} d_{k} e^{i \alpha_{k} x} \quad$ são polinômios trigonométricos, então $S(x)+T(x)$ é também um polinômio trigonométrico. 
De fato, $\quad S(x)+T(x)=\sum_{k=1}^{n+m} h_{k} e^{i \beta_{k} x}, \quad$ onde $\quad h_{k}=\left\{\begin{array}{c}c_{k}, 1 \leq k \leq n \\ d_{k}, n+1 \leq k \leq m\end{array} \quad \mathrm{e}\right.$ $\beta_{k}=\left\{\begin{array}{c}\lambda_{k}, 1 \leq k \leq n \\ \alpha_{k}, n+1 \leq k \leq m\end{array}\right.$.

Como podemos escrever $\quad c_{k}=a_{k}+i b_{k}, \quad a_{k}, b_{k} \in \Re, \quad$ e como $e^{i \lambda_{k} x}=\cos \lambda_{k} x+i \sin \lambda_{k} x$, temos que:

$$
\begin{aligned}
& \operatorname{Re}\{T(x)\}=\sum_{k=1}^{n}\left(a_{k} \cos \lambda_{k} x-b_{k} \sin \lambda_{k} x\right), \mathrm{e} \\
& \operatorname{Im}\{T(x)\}=\sum_{k=1}^{n}\left(b_{k} \cos \lambda_{k} x+a_{k} \sin \lambda_{k} x\right) .
\end{aligned}
$$

Assim, como $\operatorname{Re}\{T(x)\}=\frac{T(x)+\overline{T(x)}}{2}$ e $\operatorname{Im}\{T(x)\}=\frac{T(x)-\overline{T(x)}}{2}$, temos que as partes imaginária e real de um polinômio trigonométrico complexo são também polinômios trigonométricos complexos.

Uma função $f: \Re \rightarrow C$, onde $C$ é o conjunto dos números complexos, definida em $-\infty<x<\infty$ é chamada quase-periódica, se para qualquer $\varepsilon \geq 0$, existe um polinômio trigonométrico complexo $T_{\varepsilon}(x)$ tal que:

$$
\left|f(x)-T_{\varepsilon}(x)\right| \leq \varepsilon, \quad-\infty<x<\infty .
$$

Da definição acima, temos que qualquer polinômio trigonométrico complexo é uma função quase-periódica.

Afirmação 0 - Uma função quase-periódica é contínua e limitada na reta real.

Demonstração:

Seja $f(x)$ uma função quase-periódica e T(x) um polinômio trigonométrico tal que:

$$
\left|f(x)-T_{n}(x)\right|<\frac{1}{n},-\infty<x<\infty .
$$

A sequiência $T_{n}(x)$ converge uniformemente para a função $f(x)$ em toda a reta real. Sabemos que o limite de uma seqüência uniformemente convergente de funções 
contínua é uma função contínua. Como os polinômios trigonométricos são funções contínuas, então $f(x)$ é contínua em todo ponto da reta real.

Para mostrar que $f(x)$ é limitada, usamos o fato de que $\left|T_{1}(x)\right| \leq M^{2}$, para algum $M \in \Re$. Então, temos que:

$$
|f(x)| \leq\left|f(x)-T_{1}(x)\right|+\left|T_{1}(x)\right| \leq M+1,-\infty<x<\infty .
$$

Afirmação 1 - Uma função quase-periódica é uniformemente contínua na reta real.

Demonstração:

Primeiro, observamos que qualquer polinômio trigonométrico é uma função uniformemente contínua em $\Re$. Qualquer função $c_{k} e^{i \lambda_{k} x}$ é uniformemente contínua e consequentemente a soma de um número finito de tais funções será também uniformemente contínua.

Considere uma função quase-periódica $f(x)$ e seja $\varepsilon>0$. Existe um polinômio trigonométrico $T_{\varepsilon}(x)$ tal que:

$\left|f(x)-T_{\varepsilon}(x)\right|<\frac{\varepsilon}{3},-\infty<x<\infty$.

Da continuidade uniforme de $T_{\varepsilon}(x)$, seque que existe um número $\delta(\varepsilon)>0$ tal que:

$$
\left|T_{\varepsilon}\left(x_{1}\right)-T_{\varepsilon}\left(x_{2}\right)\right|<\frac{\varepsilon}{3}, \quad \text { se }\left|x_{1}-x_{2}\right|<\delta \text {. }
$$

Como:

${ }^{2}$ Como $T_{1}(x)$ é um polinômio trigonométrico, segue que $T_{1}(x)$ pode ser escrito como $T_{1}(x)=\sum_{k=1}^{n} c_{k} e^{i \lambda_{k} \cdot x} \cdot \operatorname{Assim},\left|T_{1}(x)\right|=\left|\sum_{k=1}^{n} c_{k} e^{i \lambda_{k} x}\right| \leq \sum_{k=1}^{n}\left|c_{k} e^{i \lambda_{k} x}\right|=\sum_{k=1}^{n}\left|c_{k}\right| \leq M$ 
$\left|f\left(x_{1}\right)-f\left(x_{2}\right)\right| \leq\left|f\left(x_{1}\right)-T_{\varepsilon}\left(x_{1}\right)\right|+\left|T_{\varepsilon}\left(x_{1}\right)-T_{\varepsilon}\left(x_{2}\right)\right|+\left|T_{\varepsilon}\left(x_{2}\right)-f\left(x_{2}\right)\right|$, segue que

$\left|f\left(x_{1}\right)-f\left(x_{2}\right)\right| \leq \varepsilon$, se $\left|x_{1}-x_{2}\right|<\delta$.

Assim, $f(x)$ é uniformemente contínua.

Afirmação 2 - O limite de seqüências uniformemente convergentes de funções quaseperiódicas é uma função quase-periódica.

Demonstração:

Seja $\left\{f_{n}(x)\right\}$ uma seqüência de funções quase-periódicas convergindo uniformemente na reta real para a função $f(x)$. Se $\varepsilon>0$, então temos que:

$\left|f(x)-f_{n}(x)\right|<\frac{\varepsilon}{2}, \quad-\infty<x<\infty, \quad n \geq N(\varepsilon)$

Por sua vez, como $f_{N}(x)$ é quase-periódica, existe um polinômio trigonométrico $T_{\varepsilon}(x)$ tal que:

$\left|f_{N}(x)-T_{\varepsilon}(x)\right|<\frac{\varepsilon}{2}, \quad-\infty<x<\infty$.

Das duas desiguladades, obtemos, utilizando a desigualdade triangular, que:

$\left|f(x)-T_{\varepsilon}(x)\right|<\varepsilon, \quad-\infty<x<\infty$.

A definição de quase-periodicidade dada anteriormente não é a defínição mais intuitiva. A definição dada por Bohr é a de que uma função $f(t), f: \Re \rightarrow C$, é quaseperiódica, se para todo $\varepsilon>0$ existir um conjunto relativamente denso de $\varepsilon$-quaseperíodos, isto é, $f(t)$ é quase-periódica se, para todo $\varepsilon>0$ existir um número $l=l(\varepsilon)>0$ tal que em cada intervalo de comprimento $l$ contido em $I$ exista pelo menos 
um número $\xi$ tal que $|f(t+\xi)-f(t)|<\varepsilon$, para $t \in I$. O número $\xi$ é chamado de um $\varepsilon$-quase-período.

Vamos mostrar que as duas definições de quase-periodicidade acima são equivalentes. Denominaremos tais funções indistintamente de funções quase-periódicas ou de funções Bohr quase-periódicas.

Daqui em diante vamos supor sempre que $f(x)$ é contínua em toda a reta real.

Por hora, vamos mostrar que as funções quase-periódicas possuem as seguintes propriedades:

A. De qualquer seqüência da forma $\left\{f\left(x+h_{n}\right)\right\}$, onde $h_{n}$ são números reais e $x+h_{n} \in I$, é possível extrair uma subseqüência uniformemente convergente na reta real.

B. Para todo $\varepsilon>0$ existe um número $l=l(\varepsilon)>0$ tal que em cada intervalo de comprimento $l$ existe pelo menos um número $\xi$ tal que $|f(t+\xi)-f(t)|<\varepsilon$, para $t \in \Re$.

Uma função que satisfaz a propriedade A é chamada função normal. Uma função que satisfaz a propriedade B satisfaz a definição de quase-periodicidade de Bohr.

Proposição 1 - Se $f(x)$ é uma função quase-periódica, então $f(x)$ satisfaz a propriedade A.

Demonstração:

Assuma que $f(x)=e^{i \lambda x}$, sendo $\lambda$ um número real. Se $\left\{h_{n}\right\}$ é uma seqüência arbitrária de números reais, então $\left\{f\left(x+h_{n}\right)\right\}=\left\{e^{i \lambda h_{n}} e^{i x \lambda}\right\}$ contém uma subseqüência uniformemente convergente na reta real. De fato, como $\left|e^{i \lambda h_{n}}\right|=1$, então como toda seqüência limitada possui subseqüência convergente, podemos extrair uma subseqüência $\left\{h_{1 n}\right\}$ da seqüência $\left\{h_{n}\right\}$, tal que a seqüência $\left\{f\left(x+h_{1 n}\right\}\right.$ convirja. Mas, $\mid f\left(x+h_{1 n}\right)-f\left(x+h_{1 m}|=| e^{i \lambda h_{1 n}}-e^{i \lambda h_{1 m}} \mid\right.$, e de acordo com o critério de Cauchy, $\left\{f\left(x+h_{1 n}\right)\right\}$ é uniformemente convergente na reta real. Obviamente, qualquer função da forma $c e^{i \lambda x}$, sendo $c$ um número complexo, também tem a propriedade $\mathrm{A}$. 
Considere agora um polinômio trigonométrico $T(x)=\sum_{k=1}^{m} c_{k} e^{i \lambda_{k} x}$ e a seqüência de números reais $\left\{h_{n}\right\}$. Podemos extrair uma subseqüência $\left\{h_{1 n}\right\}$ tal que $\left\{c_{1} e^{i \lambda_{1}\left(x+h_{1 n}\right)}\right\}$ seja uniformemente convergente na reta real. Então, de $\left\{h_{1 n}\right\}$ extraímos outra subseqüência $\left\{h_{2 n}\right\}$ tal que $\left\{c_{2} e^{i \lambda_{1}\left(x+h_{2 n}\right)}\right\}$ seja uniformemente convergente. Procedendo dessa maneira, concluímos que existe uma subseqüência $\left\{h_{m n}\right\}$ da seqüência $\left\{h_{n}\right\}$ tal que as seqüências $\left\{c_{k} e^{i \lambda_{1}\left(x+h_{k n}\right)}\right\}, k=1,2, \ldots, \mathrm{m}$. converge uniformemente. Assim, $\left\{T\left(x+h_{m n}\right)\right\}$ é uniformemente convergente na reta real, provando assim a normalidade de qualquer polinômio trigonométrico.

Considere agora uma função quase-periódica arbitrária $f(x)$ e uma seqüência de polinômios trigonométricos $\left\{T_{n}(x)\right\}$ convergindo uniformemente para $f(x)$ na reta real. Se $\left\{h_{n}\right\}$ é uma seqüência de números reais, então é possível determinar uma subseqüência $\left\{h_{1 n}\right\}$ tal que $\left\{T_{1}\left(x+h_{1 n}\right)\right\}$ seja uniformemente convergente na reta real. Então, extraímos de $\left\{h_{1 n}\right\}$ uma subseqüência $\left\{h_{2 n}\right\}$ tal que $\left\{T_{2}\left(x+h_{2 n}\right)\right\}$ seja uniformemente convergente na reta real. Procedendo desta maneira, temos que existe uma subseqüência $\left\{h_{p n}\right\}$ para algum inteiro positivo $p$ tal que $\left\{T_{q}\left(x+h_{p n}\right)\right\}$ é uniformemente convergente na reta real para qualquer $q \leq p$. Vamos agora construir uma subseqüência diagonal $\left\{h_{p p}\right\}$ que, com exceção de um número finito de termos, é uma subseqüência de qualquer seqüência $\left\{h_{q n}\right\}$. Desse modo, $\left\{T_{n}\left(x+h_{p p}\right)\right\}$ é uniformemente convergente na reta real para qualquer $n$. Seja $\varepsilon>0$ tal que $\left|f(x)-T_{n}(x)\right|<\frac{\varepsilon}{3}, \quad-\infty<x<\infty . \quad$ Existe $\quad$ um $\quad N(\varepsilon)>0 \quad$ tal que $\left|T_{n}\left(x+h_{p p}\right)-T_{n}\left(x+h_{q q}\right)\right|<\frac{\varepsilon}{3}, \quad-\infty<x<\infty, \quad p, q \geq N(\varepsilon)$. Das duas desiguladades anteriores, temos que:

$$
\begin{aligned}
& \left|f\left(x+h_{p p}\right)-f\left(x+h_{q q}\right)\right| \leq\left|f\left(x+h_{p p}\right)-T_{n}\left(x+h_{p p}\right)\right|+\left|T_{n}\left(x+h_{p p}\right)-T_{n}\left(x+h_{q q}\right)\right|+ \\
& \left|T_{n}\left(x+h_{q q}\right)-f\left(x+h_{q q}\right)\right|<\varepsilon, \quad-\infty<x<\infty, \quad p, q \geq N(\varepsilon) .
\end{aligned}
$$


Assim, a seqüência $\left\{f\left(x+h_{p p}\right)\right\}$ é uniformemente convergente na reta real, provando assim que $f(x)$ é uma função normal.

Proposição 2 - Uma função quase-periódica $f(x)$ satisfaz a propriedade B

Demonstração:

Já sabemos que $f(x)$ satisfaz a propriedade A. Suponha, por absurdo, que $f(x)$ não tem a propriedade B. Então, existe pelo menos um $\varepsilon>0$ tal que para qualquer $l>0$, é possível determinar um intervalo $I$ de comprimento $l$ e um $t(I) \in \mathfrak{R}$ tal que para todo $\zeta \in \Re,|f(t+\zeta)-f(t)| \geq \varepsilon$. Considere um número arbitrário $h_{1}$ e um intervalo $\left(a_{1}, b_{1}\right)$ da reta real de comprimento maior que $2\left|h_{1}\right|$ e que não contém um $\varepsilon$-quase-período de $f(x)$. Denote por $h_{2}=\frac{a_{1}+b_{1}}{2}$. Então, $h_{2}-h_{1} \in\left(a_{1}, b_{1}\right)$ e consequentemente $h_{2}-h_{1}$ não pode ser um $\varepsilon$-quase-período de $f(x)$. Então, existe um intervalo $\left(a_{2}, b_{2}\right)$ da reta real de comprimento maior que $2\left(\left|h_{1}\right|+\left|h_{2}\right|\right)$ e que não contém um $\varepsilon$-quase-período de $f(x)$. Fazendo $h_{3}=\frac{a_{2}+b_{2}}{2}$, temos que $h_{3}-h_{2}, h_{3}-h_{1}, h_{2}-h_{1}$ não são $\varepsilon$-quase-períodos de $f(x)$. Procedendo da mesma maneira, definimos os números $h_{4}, h_{5}, \ldots$, tais que nenhuma das diferenças $h_{i}-h_{j}, i \neq j$, são $\varepsilon$-quase-períodos de $f(x)$. Assim, para quaisquer $i$ e $j$, $\sup _{|x-y|=h_{i}-h_{j}}|f(x)-f(y)|=\sup \left|f\left(x+h_{i}\right)-f\left(x+h_{j}\right)\right|=\sup \left|f\left(x+h_{i}-h_{j}\right)-f(x)\right| \geq \varepsilon$, o que prova que a seqüência $\left\{f\left(x+h_{n}\right)\right\}$ não pode conter nenhuma subseqüência uniformemente convergente, o que contradiz o fato de $f(x)$ ser normal. Logo a normalidade de $f(x)$ implica que $f(x)$ possui a propriedade B.

Assim, se $f(x)$ é uma função quase-periódica, então $f(x)$ é uma função Bohr quase-periódica. A próxima proposição nos dará a recíproca. Para demonstrá-la, enunciamos, sem demonstrar, o seguinte resultado: 
(Fórmula de Parseval) Seja $f(x)$ uma função periódica com período $2 w$. Seja $f(x) \sim \sum_{k=-\infty}^{\infty} a_{k} e^{i w_{k} x}, w_{k}=\frac{k \pi}{w}, a_{k}=\frac{1}{2 w} \int_{0}^{2 w} f(x) e^{-i w_{k} x} d x, k=0, \pm 1, \pm 2, \ldots$, a série de Fourier associada a $f(x)$. Então $\sum_{k=-\infty}^{\infty}\left|a_{k}\right|^{2}=\frac{1}{2 w} \int_{0}^{2 w}|f(x)|^{2} d x$

Proposição 3 - Se uma função $f(x)$ contínua que satisfaz a propriedade B, então $f(x)$ é uma função quase-periódica.

Demonstração:

A demonstração desta proposição é extensa e por isso será dividida em vários passos.

Passo a. Uma função contínua com a propriedade B é limitada na reta real. Tome $\varepsilon=1$ e seja $l=l(1)$ o comprimento dado na propriedade B. Se $x$ é um número real arbitrário, então existe um $\zeta$-quase-período de $f(x)$ para o qual $-x \leq \zeta \leq-x+l$, ou $0 \leq x+\zeta \leq l$. Seja $m=\sup \{|f(x)|, 0 \leq x \leq l\}$. O número $m$ existe porque $f(x)$ é contínua. Como:

$|f(x)| \leq|f(x)-f(x+\varepsilon)|+|f(x+\varepsilon)|<1+m$.

Logo $f(x)$ é limitada.

Passo b. Uma função continua com a propriedade $B$ é uniformemente contínua na reta real.

Sejam $\varepsilon>0$ e $l=l(\varepsilon / 3)$ o comprimento correspondente a $\varepsilon / 3$ de acordo com a propriedade B. A função $f(x)$ é uniformemente contínua no intervalo $-1 \leq x \leq 1+l$, pois uma função contínua é uniformemente contínua em um intervalo fechado. Assim, existe um número $\delta=\delta(\varepsilon / 3), 0<\delta<1$, tal que:

$\left|f\left(y_{1}\right)-f\left(y_{2}\right)\right|<\frac{\varepsilon}{3}$, se $\left|y_{1}-y_{2}\right|<\delta$ ou $-1 \leq y_{1}, y_{2} \leq 1+l$. 
Considere dois números reais $x_{1}, x_{2}$ tais que $\left|x_{1}-x_{2}\right|<\delta$. Então, existe um $\varepsilon / 3$-quaseperíodo de $f(x)$, digamos $\xi$, tal que $0 \leq x_{1}+\xi \leq l$. Do mesmo modo, obtemos $-1 \leq x_{2}+\xi \leq 1+l$. Assim:

$\left|f\left(x_{1}\right)-f\left(x_{2}\right)\right| \leq\left|f\left(x_{1}\right)-f\left(x_{1}+\zeta\right)\right|+\left|f\left(x_{1}+\zeta\right)-f\left(x_{2}+\zeta\right)\right|+\left|f\left(x_{2}+\zeta\right)-f\left(x_{2}\right)\right|<\varepsilon$, desde que $x_{1}+\zeta, x_{2}+\zeta$ pertençam ao intervalo $[-1,1+l]$, e $\left|\left(x_{1}+\zeta\right)-\left(x_{2}+\zeta\right)\right|<\delta$. Segue que $f(x)$ é uniformemente contínua na reta real.

Passo $c$. Se $f(x)$ é contínua e possui a propriedade B, então para qualquer $\varepsilon>0$ existem dois números $l(\varepsilon)>\delta(\varepsilon)>0$ com a propriedade de que qualquer intervalo de comprimento l da reta real contém um subintervalo de comprimento $\delta$ cujos números contidos em tal subintervalo são $\varepsilon$-quase-periodos de $f(x)$.

Suponha $\varepsilon>0$ e seja $l_{1}=l(\varepsilon / 2)$ o comprimento correspondente a $\varepsilon / 2$ de acordo com B. Como provado no passo b, existe um número positivo $\delta_{1}=\delta(\varepsilon / 2)$ para o qual:

$|f(x+h)-f(x)|<\frac{\varepsilon}{2}, \quad-\infty<x<\infty$, se $|h|<\delta_{1}$.

Considere um $\varepsilon / 2$-quase-período, digamos $\zeta$, da função $\mathrm{f}(\mathrm{x}) . \zeta$ pertence a um certo intervalo $\alpha \leq \zeta \leq \alpha+l_{1}$. Note que para $|h|<\delta_{1}, \zeta+h \in\left[\alpha-\delta_{1} \cdot \alpha+l_{1}+\delta_{1}\right]$. Mas, $|f(x+\zeta+h)-f(x)| \leq|f(x+\zeta+h)-f(x+h)|+|f(x+h)-f(x)|<\varepsilon,-\infty<x<\infty$.

Desse modo, $\xi+h$ é um $\varepsilon$-quase-período de $f(x)$. Assim, podemos tomar $l=l_{1}+2 \delta_{1}$ e $\delta=2 \delta_{1}$. 
Passo $d$. Abaixo vamos assumir que $\varepsilon>0$. Sejam $l$ e $\delta$ os números encontrados no passo c. Considere os intervalos $I_{k}=[k l,(k+1) l], k=0, \pm 1, \pm 2, \ldots$ Em cada intervalo $I_{k}$, existe um subintervalo $\Delta_{k}=\left\lfloor\zeta_{k}-\delta / 2, \zeta_{k}+\delta / 2\right\rfloor$, tal que qualquer número em $\Delta_{k}$ é um $\varepsilon$ - quase-período da função $f(x)$.

Definimos uma função $K(s)$ na reta real como:

$K(s)=\left\{\begin{array}{l}\frac{3 l}{\delta}, \text { se } s \in \bigcup_{k=-\infty}^{\infty} \Delta_{k} \\ 0, \text { se } s \notin \bigcup_{k=-\infty}^{\infty} \Delta_{k}\end{array}\right.$.

Se $n$ é um número natural e $f_{n}(x)=\frac{1}{(6 n l)^{2}} \int_{-n l}^{n l} \int_{-n l}^{n l} f(x+s+t) K(s) K(t) d s d t$, então $\left|f(x)-f_{n}(x)\right|<2 \varepsilon, \quad-\infty<x<\infty$.

De fato,

$$
\begin{aligned}
& \frac{1}{(6 n l)} \int_{-n l}^{n l} K(s) d s=\frac{1}{(6 n l)} \sum_{k=-n}^{n-1} \frac{3 l}{\delta} \int_{\Delta_{k}} d s=\frac{1}{(2 n \delta)} \sum_{k=-n_{\Delta_{k}}}^{n-1} \int_{d} d s=\frac{1}{(2 n \delta)} 2 n \delta=1, \mathrm{e} \\
& \frac{1}{(6 n l)^{2}} \int_{-n l}^{n l} \int_{-n l}^{n l} K(s) K(t) d s d t=\left\{\frac{1}{6 n l} \int_{-n l}^{n l} K(s) d s\right\}^{2}=1 .
\end{aligned}
$$

Assim,

$$
f_{n}(x)-f(x)=\frac{1}{(6 n l)^{2}} \int_{-n l}^{n l} \int_{-n l}^{n l}(f(x+s+t)-f(x)) K(s) K(t) d s d t
$$

Supondo $K(s) K(t) \neq 0$, segue que $s, t \in \bigcup_{k=-\infty}^{\infty} \Delta_{k}$, em outras palavras, $s$ e $t$ são $\mathcal{E}$-quaseperíodos para $f(x)$. Consequentemente, 
$|f(x+s+t)-f(x)| \leq|f(x+s+t)-f(x+s)|+|f(x+s)-f(x)|<2 \varepsilon,-\infty<x<\infty$.

Assim,

$\left|f(x)-f_{n}(x)\right|<2 \varepsilon,-\infty<x<\infty$, para qualquer número natural $n$. Isso demonstra o passo d.

Passo e. Para qualquer número natural n definimos duas funções periódicas $\bar{f}_{n}(x)$ e $K_{n}(x)$ com período $6 n l$ da seguinte maneira:

$$
\begin{gathered}
\left\{\begin{array}{c}
\bar{f}_{n}(x)=f(x), \quad-3 n l \leq x \leq 3 n l \\
\bar{f}_{n}(x+6 n l)=\bar{f}(x) .
\end{array}\right. \\
\left\{\begin{array}{c}
K_{n}(x)=K(x), \quad|x| \leq n l \\
K_{n}(x)=0, \quad n l \leq|x| \leq 3 n l \text { e } x=3 n l \\
K_{n}(x+6 n l)=K_{n}(x)
\end{array}\right.
\end{gathered}
$$

Sejam,

$$
\begin{aligned}
& \bar{f}_{n}(x) \sim \sum_{k=-\infty}^{\infty} A_{n, k} e^{i w_{n, k} x}, \\
& K_{n}(x) \sim \sum_{k=-\infty}^{\infty} a_{n, k} e^{i w_{n, k} x},
\end{aligned}
$$

As séries de Fourier das funções $\bar{f}_{n}(x)$ e $K_{n}(x)$.

Os coeficientes das séries de Fourier $A_{n, k}$ e $a_{n, k}$ satisfazem plenamente as seguintes condições:

(i) $\quad \sum_{k=-\infty}^{\infty}\left|A_{n, k}\right|^{2} \leq M^{2}$

(ii) $\quad \sum_{k=-\infty}^{\infty}\left|a_{n, k}\right|^{2}=\frac{3 l}{\delta}$, 
(iii) $\left|a_{n, k}\right| \leq \min \left\{1, \frac{2}{\delta\left|w_{n, k}\right|}\right\}$,

onde $M=\sup |f(x)|, \quad-\infty<x<\infty$ e $\delta$ é aquele encontrado no passo $c$.

A desigualdade em (i) é uma aplicação direta da fórmula de Parseval na função periódica $\bar{f}_{n}(x)$. A equação em (ii) é também uma aplicação direta da fórmula de Parseval na função periódica $K_{n}(x)$. Para demonstrar (iii), observe que:

$$
\begin{aligned}
& \left|a_{n, k}\right| \leq\left|\frac{1}{6 n l} \int_{-3 n l}^{3 n l} K_{n}(x) e^{i w_{n, k} x} d x\right| \leq \frac{1}{6 n l} \int_{n l}^{n l} K(x) d x=\frac{1}{2 n l} \sum_{j=-n_{\Delta_{j}}}^{n-1} \int d x=1, \\
& \left|a_{n, k}\right|=\frac{1}{6 n l}\left|\sum_{j=-n}^{n-1} \frac{3 l}{\delta} \int_{\Delta_{j}} e^{-i w_{n, k} \cdot x} d x\right|=\frac{1}{2 n \delta}\left|\sum_{j=-n_{\xi_{j}}-\delta / 2}^{n-1} \int^{\xi_{j}+\delta / 2} e^{-i w_{n, k} \cdot x} d x\right|=\frac{1}{2 n \delta\left|w_{n, k}\right|} \mid \sum_{j=-n}^{n-1}\left\{e^{-i w_{n, k}\left(\xi_{j}+\delta / 2\right.}-e^{-i w_{n, k}\left(\xi_{j}-\delta / 2\right.}\right\} \leq \\
& \leq \frac{4 n}{2 n \delta\left|w_{n, k}\right|}=\frac{2}{\delta\left|w_{n, k}\right|}
\end{aligned}
$$

Temos assim o resultado. Note que uma conseqüência da desigualdade (i) é que:

$\left|A_{n, k}\right| \leq M$

Passo $f$. Se $|x|<n l$, então $f_{n}(x)$ pode ser expandidas pelas séries $f_{n}(x)=\sum_{k=-\infty}^{\infty} A_{n, k} a_{n,-k}^{2} e^{i w_{n, k} x}$, sendo a convergência absoluta e uniforme. Considere a função auxiliar $f_{n}^{*}(x)=\frac{1}{(6 n l)^{2}} \int_{-3 n l}^{3 n l} \int_{-3 n l}^{3 n l} \bar{f}_{n}(x+s+t) K_{n}(s) K_{n}(t) d s d t$. Claramente, essa é uma periódica com período $6 n l$. Como $K_{n}(s)=0$ para $n l \leq|s| \leq 3 n l$, segue que $f_{n}^{*}(x)=\frac{1}{(6 n l)^{2}} \int_{-n l}^{n l} \int_{-n l}^{n l} \bar{f}_{n}(x+s+t) K(s) K(t) d s d t$. Mas, para $|x|,|s|,|t|<n l$, temos que 
$|x+s+t|<3 n l$, e assim $\bar{f}_{n}(x+s+t)=f(x+s+t)$. Deste modo, para $|x|<n l$ temos que:

$f_{n}^{*}(x)=\frac{1}{(6 n l)^{2}} \int_{-n l}^{n l} \int_{-n l}^{n l} f(x+s+t) K(s) K(t) d s d t=f_{n}(x)$.

Vamos calcular os coeficientes da série de Fourier associada à função $f_{n}^{*}(x)$. Seja $f_{n}^{*}(x) \sim \sum_{k=-\infty}^{\infty} b_{n, k} e^{i w_{n, k} \cdot x}$. Temos que:

$$
\begin{aligned}
& b_{n, k}=\frac{1}{6 n l} \int_{-3 n l}^{3 n l} f_{n}^{*}(x) e^{-i w_{n, k} x} d x=\frac{1}{(6 n l)^{3}} \int_{-3 n l}^{3 n l}\left[\int_{-n l}^{n l} \int_{-n l}^{n l} \bar{f}_{n}(x+s+t) K_{n}(s) K_{n}(t) d s d t\right] e^{-w_{n, k} x} d x= \\
& =\frac{1}{(6 n l)^{3}} \int_{-3 n l}^{3 n l} \int_{-3 n l}^{3 n l}\left(\int_{-3 n l}^{3 n l} \bar{f}_{n}(x+s+t) e^{-w_{n, k} x} d x\right) K_{n}(s) K_{n}(t) d s d t
\end{aligned}
$$

Fazendo $x+s+t=u$,

$$
\begin{aligned}
& \int_{-3 n l}^{3 n l} \bar{f}_{n}(x+s+t) e^{-w_{n, k} x} d x=e^{-i w_{n, k}(-s-t)} \int_{-3 n l+s+t}^{3 n l+s+t} \bar{f}_{n}(u) e^{-w_{n, k} u} d u \text {. Logo, } \\
& b_{n, k}=\frac{1}{(6 n l)^{3}} \int_{-3 n l}^{3 n l} \int_{-3 n l}^{3 n l}\left(\int_{-3 n l}^{3 n l} \bar{f}_{n}(u) e^{-i w_{n, k} u} d u\right) e^{-i w_{n, k}(-s-t)} K_{n}(s) K_{n}(t) d s d t= \\
& =\frac{1}{(6 n l)^{3}}\left(\int_{-3 n l}^{3 n l} \bar{f}_{n}(u) e^{-w_{n, k} u} d u\right)\left(\int_{-3 n l}^{3 n l} K_{n}(s) e^{i w_{n, k} s} d s\right)\left(\int_{-3 n l}^{3 n l} K_{n}(t) e^{i w_{n, k} t} d t\right)=A_{n, k} a_{n,-k}^{2}
\end{aligned}
$$

Como $\left.\left|A_{n, k} a_{n,-k}^{2}\right| \leq\left|A_{n, k}\right|\left|a_{n,-k}\right| \leq\left.\frac{1}{2}|| A_{n, k}\right|^{2}+\left|a_{n,-k}\right|^{2}\right)$, por (i) e (ii) do passo e, a série $\sum_{k=-\infty}^{\infty}\left|b_{n, k}\right|$ é convergente. Assim, a série de Fourier de $f_{n}^{*}(x)$ é uniformemente convergente e consequentemente sua soma é $f_{n}^{*}(x)$. Ainda, como 
$f_{n}^{*}(x)=\frac{1}{(6 n l)^{2}} \int_{-n l}^{n l} \int_{-n l}^{n l} f(x+s+t) K(s) K(t) d s d t=f_{n}(x), \quad$ temos que, para $|x|<n l$, $f_{n}(x)=\sum_{k=-\infty}^{\infty} A_{n, k} a_{n,-k}^{2} e^{i w_{n, k} x}$.

Passo g. Existe um número positivo $\lambda(\varepsilon)$ independente de $n$, tal que $\left|f_{n}(x)-\sum_{\left|w_{n, k}\right| \leq \lambda} A_{n, k} a_{n,-k}^{2} e^{i w_{n, k} x}\right|<\varepsilon,|x|<n l$. Primeiramente, vamos mostrar que é possível encontrar um número positivo $\lambda(\varepsilon)$ tal que:

$\sum_{\left|w_{n, k}\right|>\lambda}\left|A_{n, k}\right|\left|a_{n,-k}^{2}\right|<\varepsilon$

Como $\left|w_{n, k}\right|>\lambda$, segue de (iii) do passo e que $\left|a_{n, k}\right|<\frac{2}{\delta \lambda}$. Além disso, como $l$ e $\delta$ dependem somente de $\varepsilon$, e $M$ é uma constante, temos:

$\sum_{\left|w_{n, k}\right|>\lambda}\left|A_{n, k}\right|\left|a_{n,-k}^{2}\right|=\sum_{\left|w_{n, k}\right|>\lambda}\left|A_{n, k}\right|\left|a_{n,-k}\right|\left|a_{n,-k}\right|<\frac{2}{\delta \lambda} \sum_{\left|w_{n, k}\right|>\lambda}\left|A_{n, k}\right|\left|a_{n,-k}\right| \leq \frac{2}{\delta \lambda}\left(\sum_{k=-\infty}^{\infty}\left|A_{n, k}\right|^{2}\right)^{1 / 2}\left(\sum_{k=-\infty}^{\infty}\left|a_{n, k}\right|^{2}\right)^{1 / 2} \leq$
$\leq \frac{2 \sqrt{3} M \sqrt{l}}{\delta^{3 / 2} \lambda}<\mathcal{E}$ quando $\lambda>\frac{2 \sqrt{3} M \sqrt{l}}{\delta^{3 / 2} \mathcal{E}}=\lambda(\varepsilon)$

Do resultado obtido no passo $f$, segue que para $|x|<n l$, temos que:

$f_{n}(x)-\sum_{\left|w_{n, k}\right| \leq \lambda} A_{n, k} a_{n,-k}^{2} e^{i w_{n, k} x}=\sum_{\left|w_{n, k}\right|>\lambda} A_{n, k} a_{n,-k}^{2} e^{i w_{n, k} x}$.

Assim,

$\left|f_{n}(x)-\sum_{\left|w_{n, k}\right| \leq \lambda} A_{n, k} a_{n,-k}^{2} e^{i w_{n, k} x}\right|=\left|\sum_{\left|w_{n, k}\right|>\lambda} A_{n, k} a_{n,-k}^{2} e^{i w_{n, k} x}\right|<\sum_{\left|w_{n, k}\right|>\lambda}\left|A_{n, k}\right|\left|a_{n,-k}^{2}\right|<\varepsilon$. 
Logo, temos o resultado.

Passo $h$. Fixemos agora o número $n$ e consideremos os $k$ 's para os quais $w_{n, k} \leq \lambda$. Obviamente, existe um número finito de inteiros $k^{\prime} s$ que satisfazem $w_{n, k} \leq \lambda$. Ordenamos os números $A_{n, k}$ em ordem decrescente de seus módulos, e os denotamos por $B_{n, 1}, B_{n, 2}, \ldots, B_{n, r_{n}}:\left|B_{n, q}\right| \geq\left|B_{n, q+1}\right|$. Denotaremos por $\mu_{n, 1}, \ldots, \mu_{n, r_{n}}$ os correspondentes expoentes $w_{n, k}$ na ordem estabelecida e por $\alpha_{n, 1}, \ldots, \alpha_{n, r_{n}}$ os números $a_{n,-k}$ sem a permutação. Seja $C_{n, q}=B_{n, q} \alpha_{n, q}^{2}$.

Existe um inteiro positivo $m=m(\varepsilon)$, independente de $n$, tal que:

$\left|f_{n}(x)-\sum_{q=1}^{m} C_{n, q} e^{i \mu_{n, q} x}\right|<2 \varepsilon,|x|<n l$.

Nós mostraremos que é possível selecionar um número $m$, independente de $n$, tal que:

$$
\sum_{q=m}^{r_{n}}\left|C_{n, q}\right|<\varepsilon
$$

Considere um número natural $p \leq r_{n}$. Como $\sum_{q=1}^{p}\left|B_{n, q}\right|^{2} \leq M^{2}$ e $B_{n, q}$ decresce em módulo, temos que $M^{2} \geq \sum_{q=1}^{p}\left|B_{n, q}\right|^{2} \geq p\left|B_{n, p}\right|^{2}$.

Assim,

$\left|B_{n, p}\right| \leq \frac{M}{\sqrt{p}}$.

Mas,

$\sum_{q=m}^{r_{n}}\left|C_{n, q}\right|=\left.\sum_{q=m}^{r_{n}}\left|B_{n, q}\right||| \alpha_{n, q}^{2}\left|\leq \frac{M}{\sqrt{p}} \sum_{q=m}^{r_{n}}\right| \alpha_{n, q}^{2}\left|\leq \frac{M}{\sqrt{p}} \sum_{q=-\infty}^{\infty}\right| a_{n, q}\right|^{2}<\varepsilon$, se $p>m=\left[\frac{9 l^{2} M^{2}}{\delta^{2} \varepsilon^{2}}\right]$, onde $[x]$, indica a parte inteira de $x$.

Assim, note que do resultado do passo g, podemos escrever: 
$\left|f_{n}(x)-\sum_{q=1}^{r_{n}} C_{n, q} e^{i \mu_{n, q^{x}}}\right|<\varepsilon$, para $|x|<n l$. Desse fato e sabendo que $\sum_{q=m}^{r_{n}}\left|C_{n, q}\right|<\varepsilon$, obtemos o resultado.

Passo i. Observe que das provas feitas nos passos $d$ e $h$, temos que:

$\left|f_{n}(x)-\sum_{q=1}^{m} C_{n, q} e^{i \mu_{n, q} x}\right|<4 \varepsilon$, para $|x|<n l$.

Como $\left|C_{n, q}\right| \leq\left|B_{n, q}\right| \leq M$ e $\left|\mu_{n, q}\right| \leq \lambda(\varepsilon)$, temos que, por sucessivas aplicações do teorema de Bolzano-Weierstrass, é possível extrair uma subseqüência de índices $n_{1}<n_{2}<\ldots<n_{k}<\ldots$ tais que:

$\lim _{k \rightarrow \infty} C_{n_{k}, q}=C_{q}, \quad \lim _{k \rightarrow \infty} \mu_{n_{k}, q}=\mu_{q}$.

Fazendo $n=n_{k}$ em $\left|f_{n}(x)-\sum_{q=1}^{m} C_{n, q} e^{i \mu_{n, q^{x}}}\right|<4 \varepsilon$, obtemos para $n_{k} \rightarrow \infty$ :

$\left|f(x)-\sum_{q=1}^{m} C_{q} e^{i \mu_{q} x}\right| \leq 4 \mathcal{E},-\infty<x<\infty$,

já que para qualquer $x$ é possível encontrar $k$ suficientemente grande para que $|x|<n_{k} l$.

Em outras palavras, dada uma função $f(x)$ com a propriedade B e um número $\varepsilon>0$, é possível determinar um polinômio trigonométrico que satisfaz $\left|f(x)-\sum_{q=1}^{m} C_{q} e^{i \mu_{q} x}\right| \leq 4 \mathcal{E}$. Isso prova a quase-periodicidade da função $f(x)$.

Obviamente que toda função contínua periódica é também uma função quase periódica. A função $f(x)=e^{i x}+e^{i \pi x}$ é um polinômio trigonométrico e, portanto, uma função quase-periódica. Tal função, porém, não é periódica. Para provarmos isso, 
suponha que exista um número real $w \neq 0$ tal que $f(x+w)=f(x)$ para qualquer $x$. Isso implicaria que:

$\left(e^{i w}-1\right) e^{i x}+\left(e^{i \pi w}-1\right) e^{i \pi x}=0$.

Sabemos, porém, que as funções $e^{i x}, e^{i \pi x}$ são linearmente independentes. Assim, $w$ deveria satisfazer $e^{i w}=e^{i \pi w}=1$. Desse modo, teríamos $w=2 k \pi, \pi w=2 h \pi, \operatorname{com} k \mathrm{e}$ $h$ inteiros. Contudo, essas duas equações são incompatíveis, logo $f(x)$ não pode ser periódica.

Outro exemplo de função quase-periódica que não é periódica é $f(x)=\cos (x)+\cos (\pi x), f: \Re \rightarrow \Re$. Note que $\cos x=\operatorname{Re}\left\{e^{i x}\right\}$ e $\cos \pi x=\operatorname{Re}\left\{e^{i \pi x}\right\}$. Como $e^{i x}$ e $e^{i \pi x}$ são polinômios trigonométricos; a parte real de um polinômio trigonométrico é também um polinômio trigonométrico, e finalmente, a soma de polinômios trigonométricos é também um polinômio trigonométrico, temos que $f(x)=\cos (x)+\cos (\pi x)$ é uma função quase-periódica. Vamos mostrar que $f(x)$ não é periódica. Se $p$ fosse um período de $f(x)$, então para qualquer $x$ teríamos:

$$
\begin{gathered}
f(x+p)-f(x)=0 \\
\cos (x+p)+\cos (\pi(x+p))-\cos (x)-\cos (\pi x)=0 \\
\cos (x) \cos (p)-\operatorname{sen}(x) \operatorname{sen}(p)+\cos (\pi x) \cos (\pi p)-\operatorname{sen}(\pi x) \operatorname{sen}(\pi p)-\cos (x)-\cos (\pi x)=0 \\
\cos (x)(\cos (p)-1)-\operatorname{sen}(x) \operatorname{sen}(p)+\cos (\pi x)(\cos (\pi p)-1)-\operatorname{sen}(\pi x) \operatorname{sen}(\pi p)=0
\end{gathered}
$$

Mas, $\cos (x), \operatorname{sen}(x), \cos (\pi x), \operatorname{sen}(\pi x)$ são linearmente independentes. De fato, considere $g(x)=A \cos (x)+B \operatorname{sen}(x)+C \cos (\pi x)+D \operatorname{sen}(\pi x), A, B, C, D \in \Re$. Supondo $g(x)=0$, vem que:

$$
\begin{aligned}
& \frac{d g(x)}{d x}=-A \operatorname{sen}(x)+B \cos (x)-\pi C \operatorname{sen}(\pi x)+\pi D \cos (\pi x) \\
& \frac{d^{2} g(x)}{d x^{2}}=-A \cos (x)-B \operatorname{sen}(x)-\pi^{2} C \cos (\pi x)-\pi^{2} D \operatorname{sen}(\pi x) \\
& \frac{d^{3} g(x)}{d x^{3}}=A \operatorname{sen}(x)-B \cos (x)+\pi^{3} C \operatorname{sen}(\pi x)-\pi^{3} D \cos (\pi x)
\end{aligned}
$$


Como as quatro igualdades acima valem para qualquer $x$, tomamos $x=0$. Desse modo temos que:

$$
\left\{\begin{array}{c}
A+C=0 \\
B+\pi D=0 \\
-A-\pi^{2} C=0 \\
-B-\pi^{3} D=0
\end{array}\right.
$$

Resolvendo o sistema acima, temos que $B=D=0$. Da equação $(\bullet)$, temos que:

$$
\left\{\begin{array}{c}
\cos p=1 \\
\operatorname{sen} p=0 \\
\cos \pi p=1 \\
\operatorname{sen} \pi p=0
\end{array}\right.
$$

Logo, $p=k \pi$ e $\pi p=k \pi$. Como essas duas igualdades são um absurdo, temos que $f(x)$ não pode ser periódica.

Dizemos que uma função $\phi(t)$ é uma função contínua por partes quase-periódica se $\phi(t)$ pertence a $P C$ (conjunto das funções contínuas por partes) e é quase-periódica.

Uma função $\phi \in P C$ é chamada de $\mathcal{E}$-oscilatória para um dado $\mathcal{E}>0$, se existe uma função contínua por partes periódica ou quase-periódica $\psi(t)$ tal que $|\psi(t)-\phi(t)|<\mathcal{E}$ para todo $t \geq 0$.

Uma solução $\psi$ de (3) (ou de (4)) é dita estável se para um dado $\varepsilon>0$, existe um $\delta>0$ tal que qualquer outra solução $\varphi$ de (3) (ou de (4)) que satisfaz $|\varphi(0)-\psi(0)|<\delta$ também satisfaz $|\varphi(t)-\psi(t)|<\mathcal{E}$ para todo $t$ no domínio de (3) ou de (4). A solução $\psi$ de (3) (ou de (4)) é dita assimptoticamente estável se além de ser estável satisfaz $|\varphi(t)-\psi(t)| \rightarrow 0$, quando $t \rightarrow \infty$.

Considere o seguinte sistema:

$$
\frac{d x}{d t}=A x, t \neq \tau_{i},\left.\Delta x\right|_{t=\tau_{i}}=B x(7),
$$


onde $A$ e $B$ são matrizes constantes e os instantes de salto $\tau_{i}$ são indexados por números naturais em ordem crescente tais que $\tau_{i} \rightarrow \infty$ quando $i \rightarrow \infty$.

Utilizando a decomposição de Jordan, vamos assumir que a matriz $A$ está escrita na forma canônica real, isto é, $A=\operatorname{diag}\left\{D_{1}, \ldots, D_{m}\right\}$, onde $D_{j}=\lambda_{j} I d+\varepsilon_{1} W$, $W=\left(\begin{array}{cccccc}0 & 1 & 0 & \ldots & 0 & 0 \\ 0 & 0 & 1 & \ldots & 0 & 0 \\ \ldots & \ldots & \ldots & \ldots & \ldots & \ldots \\ 0 & 0 & 0 & \ldots & 0 & 1 \\ 0 & 0 & 0 & \ldots & 0 & 0\end{array}\right), \lambda_{j}$ são os auto-valores de $A$ e $I d$ é a matriz identidade. Vamos assumir que o parâmetro $\varepsilon_{1}$ que caracteriza esta forma é suficientemente pequeno.

De acordo com o teorema 1, qualquer solução do sistema (7), $x(t), x\left(t_{0}, x_{0}\right)=x_{0}$ é dada pela fórmula $x\left(t, x_{0}\right)=X\left(t, t_{0}\right) x_{0}$, onde $X\left(t, t_{0}\right)=e^{A\left(t-\tau_{i}\right)} \prod_{t_{0}<\tau_{j}<\tau_{i}}(I d+B) e^{A\left(\tau_{j}-\tau_{j-1}\right)}$, $\tau_{0}=t_{0}, \tau_{i}<t<\tau_{i+1}$.

Os resultados a seguir serão importantes para a demonstração do teorema que garantirá, sob algumas condições, a existência de solução para o caso quase-periódico. Tal teorema garantirá, também sob certas condições, que a solução obtida será assintoticamente estável.

Lema 1 - Sejam $A$ uma matriz constante na forma canônica real, $\lambda_{1}, \ldots, \lambda_{n}$ seus autovalores, $\varepsilon_{1}>0$, e $\gamma=\max \left\{\operatorname{Re} \lambda_{j}\right\}, j=1, \ldots, n$. Então, para todo $t \geq 0$, temos que:

$$
\left\|e^{A t} x\right\| \leq e^{\left(\varepsilon_{1}+\gamma\right) t}\|x\| \text {, onde }\|x\|^{2}=\langle x, x\rangle=\sum_{j=1}^{n} x_{j}^{2} .
$$

Demonstração:

Suponha, inicialmente, que $A=\lambda I d+\varepsilon W$. Então, 
$e^{A t}=\sum_{n=0}^{\infty} \frac{t^{n}}{n !} \sum_{k=0}^{n}\left(\begin{array}{l}n \\ k\end{array}\right) \varepsilon^{k} \lambda^{n-k} W^{k}$. É fácil verificar que essa série é convergente. Logo, podemos escrever:

$e^{A t}=\sum_{k=0}^{n} \sum_{n=k}^{\infty}\left(\begin{array}{l}n \\ k\end{array}\right) \frac{t^{n}}{n !} \lambda^{n} \varepsilon^{k} \lambda^{-k} W^{k}=\sum_{k=0}^{\infty} \sum_{n=k}^{\infty}\left(\begin{array}{l}n \\ k\end{array}\right) \frac{t^{n}}{n !} \lambda^{n} \varepsilon^{k} \lambda^{-k} W^{k}$. Essa igualdade é verdadeira, pois existe um $c \in \Re$ tal que $W^{c}$ é a matriz nula. Assim,

$e^{A t}=\sum_{k=0}^{\infty} \frac{1}{k !}\left(t^{k} \lambda^{k}\right) e^{\lambda t} \lambda^{-k} \varepsilon^{k} W^{k}=e^{\lambda t} \sum_{k=0}^{c} \frac{t^{k} \varepsilon^{k}}{k !} W^{k}$. Mas $|W x| \leq|x|$. Logo,

$\left|e^{A t}\right|=\left|e^{\lambda t} \sum_{k=0}^{c} \frac{t^{k} \varepsilon^{k}}{k !} W^{k} x\right| \leq e^{\lambda t}\left(\sum_{k=0}^{c} \frac{t^{k} \varepsilon^{k}}{k !}\right)|x| \leq e^{\lambda t} e^{\varepsilon t}|x|$.

Para generalizarmos o resultado, suponha $n=2$, isto é, $A=\operatorname{diag}\left\{D_{1}, D_{2}\right\}$. Então, denotamos por $B_{i}$, a matriz que possui as mesmas dimensões que a matriz $\mathrm{A}$, e seja tal que:

$\left(\begin{array}{cccc}D_{i} & 0 & \ldots & 0 \\ 0 & 0 & \ldots & 0 \\ \ldots & \ldots & \ldots & 0 \\ 0 & 0 & 0 & 0\end{array}\right), i=1,2$. Então, $\left|e^{A t}\right| \leq\left|e^{B_{1}}\right|+\left|e^{B_{2} t}\right|$. Isso reduz o caso $\mathrm{n}=2$ ao caso em que $A=\lambda I d+\varepsilon W$. Por indução, temos o resultado.

Teorema 5 - Sejam no sistema (7) a matriz A na forma real canônica e os instantes $\tau_{i}$ tais que existe o limite $\lim _{T \rightarrow \infty} \frac{i(t, t+T)}{T}=p$, onde $i(t, t+T)$ é o número de pontos da sequiência $\left\{\tau_{i}\right\}$ que pertencem ao intervalo $[t, t+T]$. Sejam também $\gamma=\max \left\{\operatorname{Re} \lambda_{j}\right\}$ e $\alpha^{2}=\max \left\{\lambda_{j}\left((I d+B)^{T}(I d+B)\right\}\right.$, onde $T$ representa o operador conjugação e $\lambda_{j}(A)$ representa o $j$-ésimo autovalor da matriz $A$. Se valer a desigualdade $\gamma+p \ln \alpha<0$, então o sistema (7) é assimptoticamente estável.

Demonstração: 
Para quaisquer duas soluções do sistema (7), $x(t), x\left(t_{0}\right)=x_{0}$ e $y(t), y\left(t_{0}\right)=y_{0}$, temos $x(t)-y(t)=e^{A\left(t-\tau_{i}\right)} \prod_{t_{0}<\tau_{i}<t}(I d+B) e^{A\left(\tau_{j}-\tau_{j-1}\right)}\left(x_{0}-y_{0}\right)$. Do lema 1, temos que:

$\|x(t)-y(t)\|=e^{\left(\varepsilon_{1}+\gamma\right)\left(t-t_{0}\right)} \alpha^{i\left(t_{0}, t\right)}\left\|x_{0}-y_{0}\right\|, t \geq t_{0}$. Como o limite $\lim _{T \rightarrow \infty} \frac{i(t, t+T)}{T}=p$ existe, segue que para qualquer $\varepsilon_{2}>0$, existe um $K=K\left(\varepsilon_{2}\right)>0$ tal que $\alpha^{i\left(t_{0}, t\right)} \leq K e^{\left(\varepsilon_{2}+p \ln \alpha\right)\left(t-t_{0}\right)}$.

De fato, $\alpha^{i\left(t_{0}, t\right)}=\alpha^{\frac{i\left(t_{0}, t\right)}{t-t_{0}}\left(t-t_{0}\right)}=e^{\frac{i\left(t_{0}, t\right)}{t-t_{0}}\left(t-t_{0}\right) \ln \alpha}=e^{\frac{i\left(t_{0}, t\right)}{t-t_{0}} \ln \alpha\left(t-t_{0}\right)} \leq K e^{\left(\varepsilon_{2}+p \ln \alpha\right)\left(t-t_{0}\right)}$.

Consequentemente, $\|x(t)-y(t)\|=K e^{\left(\varepsilon_{1}+\varepsilon_{2}+\gamma+p \ln \alpha\right)\left(t-t_{0}\right)}\left\|x_{0}-y_{0}\right\|, t \geq t_{0}$. Isso encerra a demonstração, pois como $\gamma+p \ln \alpha<0$ e $\varepsilon_{1}, \varepsilon_{2}$ podem ser escolhidos suficientemente pequenos, temos que $\|x(t)-y(t)\| \rightarrow 0$, quando $t \rightarrow \infty$.

Teorema 6 - Para toda seqüência quase-periódica $\left\{x_{i}\right\}$, existe um valor médio finito definido por $M\left(\left\{x_{i}\right\}\right)=\lim _{n \rightarrow \infty} \frac{1}{n} \sum_{j=1}^{i+n-1} x_{j}$, que é uniforme com respeito a $i \in Z$.

\section{Demonstração:}

Inicialmente, vamos fazer uma estimativa para $\sum_{j=1}^{i+n-1} x_{j}-\sum_{j=0}^{n-1} x_{j}$. Como a seqüência é quase-periódica, para o número $\frac{\varepsilon}{4}>0$, existe um número $N=N(\varepsilon)$ tal que, entre os inteiros do intervalo $[i, i+N]$, poderia ser encontrado um $\frac{\varepsilon}{4}-$ quase-período $p$, isto é, um inteiro $p$ tal que $\left\|x_{i+p}-x_{i}\right\|<\frac{\varepsilon}{4}$ para todo $i \in Z$. Tendo escolhido $\varepsilon$ e $i$ e determinado $N$ e $p$, podemos escrever:

$$
\begin{aligned}
& \sum_{j=1}^{i+n-1} x_{j}-\sum_{j=0}^{n-1} x_{j}=\sum_{j=p}^{p+n-1} x_{j}-\sum_{j=0}^{n-1} x_{j}+\sum_{j=i}^{p-1} x_{j}-\sum_{j=i+n}^{p+n-1} x_{j} . \\
& \text { Como }\left\|x_{i+p}-x_{i}\right\|<\frac{\varepsilon}{4} \text { e } 0 \leq p-i \leq N, \text { temos que: }
\end{aligned}
$$


$\left\|\sum_{j=1}^{i+n-1} x_{j}-\sum_{j=0}^{n-1} x_{j}\right\| \leq \sum_{j=0}^{n-1}\left\|x_{j+p}-x_{j}\right\|+\sum_{j=i}^{p-1}\left\|x_{j}\right\|+\sum_{j=i+n}^{p+n-1}\left\|x_{j}\right\| \leq \frac{\varepsilon}{4} n+2 N \sup _{i \in Z}\left\|x_{i}\right\|$

Vamos mostrar que a seqüência $\left\{y_{k}\right\}, y_{k}=\frac{1}{k} \sum_{j=0}^{k-1} x_{j}$ tem limite para $k \rightarrow \infty$. Para isso vamos usar o critério de Cauchy. Para quaisquer números naturais $n$ e $m$ temos:

$$
\begin{aligned}
& \left\|\frac{1}{m} \sum_{j=0}^{m-1} x_{j}-\frac{1}{n} \sum_{j=0}^{n-1} x_{j}\right\| \leq \frac{1}{m n}\left\|n \sum_{j=0}^{m-1} x_{j}-\sum_{j=0}^{m n-1} x_{j}\right\|+\frac{1}{m n}\left\|\sum_{j=0}^{m n-1} x_{j}-m \sum_{j=0}^{n-1} x_{j}\right\| \leq \\
& \frac{1}{m n} \sum_{v=1}^{n}\left\|\sum_{j=0}^{m-1} x_{j}-\sum_{j=(v-1) m}^{m v-1} x_{j}\right\|+\frac{1}{m n} \sum_{v=1}^{m}\left\|\sum_{j=(v-1) n}^{m-1} x_{j}-\sum_{j=0}^{n-1} x_{j}\right\| .
\end{aligned}
$$

Usando a estimativa anterior, temos que:

$\left\|\frac{1}{m} \sum_{j=0}^{m-1} x_{j}-\frac{1}{n} \sum_{j=0}^{n-1} x_{j}\right\| \leq \frac{1}{m n} n\left(\frac{\varepsilon}{4} m+2 N \sup _{i \in Z}\left\|x_{i}\right\|\right)+\frac{1}{m n} m\left(\frac{\varepsilon}{4} n+2 N \sup _{i \in Z}\left\|x_{i}\right\|\right)=\frac{\varepsilon}{2}+2 N\left(\frac{1}{m}+\frac{1}{n}\right) \sup _{i \in Z}\left\|x_{i}\right\|$ Escolhendo $N_{0}$ tal que $N_{0}>\frac{8 N}{\varepsilon} \sup _{i \in Z}\left\|x_{i}\right\|$, temos que para todos $m>N_{0}, n>N_{0}$, $\left\|\frac{1}{m} \sum_{j=0}^{m-1} x_{j}-\frac{1}{n} \sum_{j=0}^{n-1} x_{j}\right\| \leq \varepsilon$

O critério de Cauchy implica que a seqüência $\left\{y_{k}\right\}$ converge, isto é $\lim _{n \rightarrow \infty} \frac{1}{n} \sum_{j=1}^{i+n-1} x_{j}=x^{0}$ existe. Finalmente, vamos mostrar que $M\left(\left\{x_{i}\right\}\right)=\lim _{n \rightarrow \infty} \frac{1}{n} \sum_{j=1}^{i+n-1} x_{j}$ existe. Note que de $\left(^{*}\right)$ temos que $\left\|\frac{1}{k} \sum_{i=(i-1) k}^{i k-1} x_{i}-\frac{1}{k} \sum_{i=0}^{k-1} x_{j}\right\| \leq \frac{\varepsilon}{4}+\frac{2 N}{k} \sup _{i \in Z}\left\|x_{i}\right\|$, para $i=1,2, \ldots$. Consequentemente, pela expressão $\frac{1}{n} \sum_{k=1}^{n}\left[\frac{1}{i} \sum_{j=(k-1) i}^{k i-1} x_{j}-\frac{1}{i} \sum_{j=0}^{i-1} x_{j}\right]=\frac{1}{n i} \sum_{j=0}^{n i-1} x_{i}-\frac{1}{i} \sum_{j=0}^{i-1} x_{i}$, temos que:

$\left\|\frac{1}{n i} \sum_{j=0}^{n i-1} x_{j}-\frac{1}{i} \sum_{j=0}^{i-1} x_{j}\right\| \leq \frac{\varepsilon}{4}+\frac{2 N}{i} \sup _{i \in Z}\left\|x_{i}\right\|$

Se aplicarmos o limite nesta última desigualdade fazendo $n \rightarrow \infty$, temos que: $\left\|x^{0}-\frac{1}{i} \sum_{j=0}^{i-1} x_{j}\right\| \leq \frac{\varepsilon}{4}+\frac{2 N}{i} \sup _{i \in Z}\left\|x_{i}\right\| \cdot$

Segue de $\left(^{*}\right)$ que $\left\|x^{0}-\frac{1}{n} \sum_{j=i}^{i+n-1} x_{j}\right\| \leq \frac{\varepsilon}{2}+\frac{4 N}{n} \sup _{i \in Z}\left\|x_{i}\right\|<\varepsilon$, para $n>\frac{8 N}{\varepsilon} \sup \left\|x_{i \in Z}\right\|$. Isto implica que $\lim _{n \rightarrow \infty} \frac{1}{n} \sum_{j=1}^{i+n-1} x_{j}=M\left(\left\{x_{i}\right\}\right)=x^{0}$. 
Lema 2 - Sejam os números $\tau_{i}, i \in Z$, tais que a sequiência $\left\{\bar{\tau}_{i}\right\}, \bar{\tau}_{i}=\tau_{i+1}-\tau_{i}, i \in Z$ seja quase-periódica. Então existe o limite $\lim _{T \rightarrow \infty} \frac{i(t, t+T)}{T}=p$, para algum $p \in \Re$. Tal convergência é uniforme com respeito a $t \in \mathfrak{R}$.

Demonstração:

Vamos mostrar inicialmente que o limite $\lim _{n \rightarrow \infty} \frac{\tau_{n}}{n}=\frac{1}{p}$ existe e é diferente de zero. De fato, sem perda de generalidade, podemos assumir que $\tau_{-1}<0$ e $\tau_{0} \geq 0$. Então, $\tau_{n}=\tau_{0}+\sum_{j=0}^{n-1} \bar{\tau}_{j}$. Logo, $\frac{\tau_{n}}{n}=\frac{\tau_{0}}{n}+\frac{1}{n} \sum_{j=0}^{n-1} \bar{\tau}_{j}$. Como a seqüência é quase-periódica, o teorema 6 implica que o limite $\lim _{n \rightarrow \infty} \frac{1}{n} \sum_{j=0}^{n-1} \bar{\tau}_{j}$ existe e é finito. Além disso, como a assumimos no item 2 do capítulo 2 que seqüência $\left\{\bar{\tau}_{i}\right\}$ é positiva, este limite é diferente de zero. Deste modo, o limite $\lim _{n \rightarrow \infty} \frac{\tau_{n}}{n}=\lim _{n \rightarrow \infty}\left(\frac{\tau_{0}}{n}+\frac{1}{n} \sum_{j=0}^{n-1} \bar{\tau}_{j}\right)=\frac{1}{p}$ existe e é diferente de zero.

De fato, a existência é dada pelo Teorema 6. Para verificarmos que tal limite é diferente de zero, vamos mostrar que se $\tau_{j} \geq 0$, então $M\left\{\tau_{j}\right\}=0$, se e somente se, $\tau_{j}=0$ para qualquer $j \in N$. Esse resultado, conjugado com o fato de que a seqüência $\left\{\tau_{j}\right\}$ é crescente, implica que $\lim _{n \rightarrow \infty} \frac{\tau_{n}}{n}>0$. Vamos supor $\tau_{1}>0$. Podemos escolher $\rho \in \Re$ com $\tau_{1}-\rho>0$. Então, dado $\frac{\rho}{2}$, existe $N_{\frac{\rho}{2}} \in N$ tal que para qualquer $i \in N$, existe $p \in\left[i, i+N_{\frac{\rho}{2}}\right]$ tal que para qualquer $k \in N$, temos que $\left|\tau_{k+p}-\tau_{p}\right|<\frac{\rho}{2}$. Começando com $i_{1}=2$, escolha $p_{1} \in\left[i_{1}, i_{1}+N_{\frac{\rho}{2}}\right]$ com $\left|\tau_{1+p_{1}}-\tau_{1}\right|<\frac{\rho}{2}$. Assim, $\tau_{1}-\frac{\rho}{2}<\tau_{1+p_{1}}$. Mas, $\tau_{1}-\rho<\tau_{1}-\frac{\rho}{2}$. Logo, $\tau_{1}-\rho<\tau_{1+p_{1}}$. Agora, seja $i_{2}=i_{1}+N_{\frac{\rho}{2}}+1$. Podemos 
escolher $p_{2} \in\left[i_{2}, i_{2}+N_{\frac{\rho}{2}}\right]$ tal que $\left|\tau_{1+p_{2}}-\tau_{1}\right|<\frac{\rho}{2}$. Note que $p_{2}-p_{1}<2\left(N_{\frac{\rho}{2}}+1\right)$. Procedendo assim, podemos encontrar $p_{3}, p_{4}, \ldots$ tais que $p_{k}-p_{1}<(k+1)\left(N_{\frac{\rho}{2}}+1\right)$, isto é, $\quad p_{k}<(k+1)\left(N_{\frac{\rho}{2}}+1\right)+p_{1}$. Logo, $\quad \frac{1}{p_{k}}>\frac{1}{(k+1)\left(N_{\frac{\rho}{2}}+1\right)+p_{1}}$. Então, $\frac{1}{p_{k}} \sum_{j=1}^{p_{k}} \tau_{j}>\frac{k\left(\tau_{1}-\rho\right)}{p_{k}}>\frac{k}{(k+1)} \frac{\left(\tau_{1}-\rho\right)}{\left(2 N_{\frac{\rho}{2}}+1\right)+p_{1}}$. Como $\lim _{k \rightarrow \infty}\left(\frac{k}{(k+1)} \frac{\left(\tau_{1}-\rho\right)}{\left(2 N_{\frac{\rho}{2}}+1\right)+p_{1}}\right)=\frac{\left(\tau_{1}-\rho\right)}{\left(2 N_{\frac{\rho}{2}}+1\right)+p_{1}}>0$, temos o resultado. Portanto, o limite $\lim _{n \rightarrow \infty} \frac{i\left(0, \tau_{n}\right)}{\tau_{n}}=p$ também existe. Agora, vamos mostrar que $\lim _{T \rightarrow \infty} \frac{i(0, T)}{T}=p$. De fato, $\frac{T}{i(0, T)}=\frac{\tau_{i}+\theta_{i}}{i}$ para algum número natural $i$ e algum $\theta_{i}$ tal que $0 \leq \theta_{i} \leq \sup _{i \in Z} \bar{\tau}_{i}$. Assim, $\frac{T}{i(0, T)}-\frac{\tau_{i}}{i}=O\left(\frac{1}{i}\right)^{3}$. Temos, do mesmo modo que fizemos na demonstração do teorema 6 , que a propriedade da seqüência $\left\{\bar{\tau}_{i}\right\}$ implica que $\left\|\sum_{j=v}^{r+i-1} \bar{\tau}_{j}-\sum_{j=0}^{i-1} \bar{\tau}_{j}\right\| \leq \frac{\varepsilon i}{4}+2 N \sup _{i \in Z} \bar{\tau}_{j}$. Usando essa desigualdade, podemos escrever: $\left\|\frac{1}{i} \sum_{j=(v-1) i}^{v i-1} \bar{\tau}_{j}-\frac{1}{i} \sum_{j=0}^{i-1} \bar{\tau}_{j}\right\| \leq \frac{\varepsilon i}{4}+\frac{2 N}{i} \sup _{i \in Z} \bar{\tau}_{j}$, para $v=1,2, \ldots$.Consequentemente, como a média aritmética é $\frac{1}{n} \sum_{v=1}^{n}\left[\frac{1}{i} \sum_{j=(v-1) i}^{v i-1} \bar{\tau}_{j}-\frac{1}{i} \sum_{j=0}^{i-1} \bar{\tau}_{j}\right]=\frac{1}{n i} \sum_{j=0}^{n i-1} \bar{\tau}_{i}-\frac{1}{i} \sum_{j=0}^{i-1} \bar{\tau}_{j}$, podemos fazer a seguinte estimativa: $\left|\frac{1}{n i} \sum_{j=0}^{n i-1} \bar{\tau}_{i}-\frac{1}{i} \sum_{j=0}^{i-1} \bar{\tau}_{j}\right| \leq \frac{\varepsilon}{4}+\frac{2 N}{i} \sup _{j \in Z} \bar{\tau}_{j}$.

${ }^{3}$ Se $F$ e $G$ são funções, vale a notação: $F(x)=O(G(x)) \Rightarrow$ Existe $M \in \Re$ tal que $\left|\frac{F(x)}{G(x)}\right| \leq M$. 
Usando as desigualdades acima obtemos:

$\left|\frac{1}{i} \sum_{i=v}^{v+i-1} \bar{\tau}_{j}-\frac{1}{p}\right| \leq \frac{\varepsilon}{2}+\frac{4 N}{i} \sup _{j \in Z} \bar{\tau}_{j}$. Se substituirmos $i$ por $n$ e $v$ por $i$, obtemos que $\lim _{n \rightarrow \infty} \frac{\tau_{i+n}-\tau_{i}}{n}=\frac{1}{p}$ uniformemente com respeito a $i \in Z$.

Suponha que o intervalo $[t, t+T]$ contenha $k$ termos da seqüência $\left\{\tau_{i}\right\}$. Sejam esses termos: $\tau_{v+1}, \tau_{v+2}, \ldots, \tau_{v+k}$. Então, $\frac{T}{i(t, t+T)}=\frac{T}{k}=\frac{\tau_{v+k}-\tau_{v}+\theta_{k}}{k},\left|\theta_{k}\right| \leq \sup _{j \in Z} \bar{\tau}_{j}$. Como $\lim _{n \rightarrow \infty} \frac{\tau_{i+n}-\tau_{i}}{n}=\frac{1}{p}$, segue que $\lim _{T \rightarrow \infty} \frac{i(t, t+T)}{T}=p$, para todo $t \in \Re$.

Sejam $\varepsilon>0$ e $\Gamma_{\varepsilon}$ o conjunto de todos os números reais $r$ para os quais existe pelo menos um número $k$ tal que $\left|\tau_{i}^{k}-r\right|<\mathcal{E}, i \in Z, \tau_{i}^{k}=\tau_{i+k}-\tau_{i}$. Vamos denotar por $\mathrm{P}_{r}$ o conjunto de todos os números $k$ que satisfazem $\left|\tau_{i}^{k}-r\right|<\varepsilon, i \in Z$, para $\varepsilon$ e $r$ fixados. Denotaremos por $\mathrm{P}_{\varepsilon}$ a união de todos os conjuntos $\mathrm{P}_{r}$ para todos os $r \in \Gamma_{\varepsilon}$, isto é, $\mathrm{P}_{\varepsilon}=\bigcup_{r \in \Gamma_{\varepsilon}} \mathrm{P}_{r}$.

Lema 3 - Se $p$ é $\varepsilon$-quase-período, comum a todas as seqüências $\left\{\tau_{i}^{j}\right\}, j \in N$ fixado, então $\tau_{0}^{p} \in \Gamma_{\varepsilon}$ e $p \in P_{\varepsilon}$. Se $p \in P_{\varepsilon}$, então $p$ é $2 \varepsilon$-quase-período, comum a toda seqüência $\left\{\tau_{i}^{j}\right\}$.

Demonstração:

Sabemos que $\tau_{i}^{p}-\tau_{0}^{p}=\tau_{p}^{i}-\tau_{0}^{i}$. Se interpretarmos a família de seqüências $\left\{\tau_{i}^{j}\right\}$ como um conjunto de seqüências em $i$, sendo tal conjunto indexado em $j$, então a seqüência $\tau_{n}^{i}$ (seqüência em $n$ ) é quase-periódica e sendo $p$ um $\varepsilon$-quase-período, temos que $\left|\tau_{p}^{i}-\tau_{0}^{i}\right|<\varepsilon$, como desejado. Desse modo, $\tau_{0}^{p} \in \Gamma_{\varepsilon}$ e $p \in P_{\tau_{i}^{p}} \subseteq P_{\varepsilon}$. 
Agora, suponha $p \in P_{\varepsilon}$. Então, existe $r \in \Gamma_{\varepsilon}$ tal que $p \in P_{r}$, isto é, para qualquer $i \in N, \quad\left|\tau_{i}^{p}-r\right|<\mathcal{E}$. Fixado $j \in N$, temos que para qualquer $i \in N$, $\left|\tau_{i+p}^{j}-\tau_{i}^{j}\right|=\left|\tau_{i+j}^{p}-\tau_{i}^{p}\right|$. Assim, $\left|\tau_{i+p}^{j}-\tau_{i}^{j}\right| \leq\left|\tau_{i+p}^{p}-r\right|+\left|r-\tau_{i}^{p}\right|<2 \varepsilon$.

Lema 4 - Sejam $\Gamma \subset \Gamma_{\varepsilon}, \Gamma \neq \phi$, e $\mathrm{P} \subseteq \underset{r \in \Gamma}{\mathrm{P}_{r}}$ com $\mathrm{P} \cap \mathrm{P}_{r} \neq \phi$ para todo $r \in \Gamma$. Então, o conjunto $\Gamma$ é relativamente denso se, e somente se, o conjunto $\mathrm{P}$ é relativamente denso.

\section{Demonstração:}

Os inteiros de $\mathrm{P}$ podem ser arranjados de modo que a seqüência $\left\{p_{k}\right\}$ seja crescente. Como a seqüência $\left\{\tau_{i}\right\}$ é crescente, a seqüência $\mathrm{P}^{\prime}=\left\{\tau_{0}^{p_{k}}\right\}$ também é crescente. Vamos mostrar inicialmente que $\Gamma$ é relativamente denso se, e somente se, $\mathrm{P}^{\prime}$ também o é.

Suponha que $\Gamma$ é relativamente denso. Queremos mostrar que $\mathrm{P}^{\prime}$ também o é, isto é, mostrar que existe $L>0$ tal que, para qualquer $x \in \Re$ temos que $[x, x+L] \cap \mathrm{P}^{\prime} \neq \varnothing$. Sabemos que $\Gamma$ é relativamente denso. Logo existe $l>0$ tal que, para qualquer $x \in \Re$ temos que $[x, x+l] \cap \Gamma \neq \varnothing$. Agora, escolha $x$ arbitrariamente. Existe $r \in \Gamma$ com $x \leq r \leq x+l$. Como $r \in \Gamma$, existe $k \in \mathrm{P}_{r}$ tal que para qualquer $i \in N$ temos $\left|\tau_{i}^{k}-r\right|<\varepsilon . \quad$ Em particular, $\quad\left|\tau_{0}^{k}-r\right|<\varepsilon$. Assim, $\quad r-\varepsilon<\tau_{0}^{k}<r+\varepsilon$. Mas, $r+\varepsilon \leq x+l+\varepsilon$ e $r-\varepsilon \geq x-\varepsilon, \log 0 x-\varepsilon<\tau_{0}^{k}<x+l+\varepsilon$. Essa última desigualdade vale para qualquer $k \in \mathrm{P}_{r}$, assim seja $k \in \mathrm{P}_{r} \cap \mathrm{P}$. Desse modo, existe $m \in N$ com $k=p_{m}$ e temos que $x-\varepsilon<\tau_{0}^{p_{m}}<x+l+\mathcal{E}$. Como $x$ é arbitrário, se fizermos $z=x-\varepsilon$, temos que $z<\tau_{0}^{p_{m}}<z+l+2 \varepsilon$, isto é $L=l+2 \varepsilon$ e, portanto, $\mathrm{P}^{\prime}$ é relativamente denso. No sentido inverso, suponha que $\mathrm{P}^{\prime}$ é relativamente denso. Queremos mostrar que $\Gamma$ é relativamente denso. Assim, escolhemos $x \in \mathfrak{R}$. Sabemos que existe $l>0$ com $[x, x+l] \cap \mathrm{P}^{\prime} \neq \varnothing$. Assim, seja $\tau_{0}^{p_{k}} \in \mathrm{P}^{\prime}$ e $x \leq \tau_{0}^{p_{k}} \leq x+l$. Como $p_{k} \in \mathrm{P}$, existe $r \in \Gamma$ tal que $p_{k} \in \mathrm{P}_{r}$, isto é, para qualquer $i \in N$ temos que $\left|\tau_{i}^{p_{k}}-r\right|<\varepsilon$. Em particular $\tau_{0}^{p_{k}}-\varepsilon<r<\tau_{0}^{p_{k}}+\varepsilon$. Deste modo, $x-\varepsilon<r<x+l+\varepsilon$. Como já feito anteriormente, 
se $z=x-\varepsilon, z<r<z+l+2 \varepsilon$. Como $x$ é arbitrário, $z$ também o é e, portanto, $\Gamma$ é relativamente denso.

Agora, vamos mostrar que $\mathrm{P}$ é relativamente denso se, e somente se, $\mathrm{P}^{\prime}$ é relativamente denso. Isso será provado se mostrarmos que:

1) $\lim _{k \rightarrow-\infty} p_{k}=-\infty$ se e somente se $\lim _{k \rightarrow-\infty} \tau_{0}^{p_{k}}=-\infty$;

2) $\lim _{k \rightarrow \infty} p_{k}=\infty$ se e somente se $\lim _{k \rightarrow \infty} \tau_{0}^{p_{k}}=\infty$;

3) A sequiência $\left\{p_{k+1}-p_{k}\right\}$ é limitada se e somente se a seqüência $\left\{\tau_{0}^{p_{k+1}}-\tau_{0}^{p_{k}}\right\}$ é limitada.

Antes de mostrarmos 1), 2) e 3), observamos que se $\left\{x_{i}\right\}$ é uma seqüência de números reais crescente, $i \in N$, com $x_{i} \underset{i \rightarrow \infty}{\rightarrow \infty}, x_{i} \rightarrow-\infty$ e $\sup \left\{x_{i+1}-x_{i}\right\}=M<\infty$, então o conjunto $\left\{x_{i}\right\}$ é relativamente denso. De fato, para um dado $x \in \mathfrak{R}$, o intervalo $[x, x+M]$ deve conter algum $x_{j}$. Caso contrário, como $\underset{i \rightarrow \infty}{x_{i \rightarrow \infty}} \rightarrow$, então existiria um máximo $p$ tal que $x_{p}<x$ e $x+M<x_{p+1}$. Mas, teríamos $x_{p+1}-x_{p}>M$, o que é uma contradição. Logo, $\left\{x_{i}\right\}$ é relativamente denso. Assim, 1), 2) e 3) valem para $\mathrm{P}$ se e somente se valerem para $\mathrm{P}^{\prime}$.

As afirmações 1) e 2) seguem diretamente das definições e propriedades das seqüências $\left\{\tau_{i}\right\},\left\{p_{k}\right\},\left\{\tau_{0}^{p_{k}}\right\}$. Resta assim provarmos a afirmação 3 ). Como $\tau_{i+k}^{j}-\tau_{i}^{j}=\tau_{i+j}^{k}-\tau_{i}^{k}$ e $\tau_{i}^{j}-\tau_{i}^{k}=\tau_{i+k}^{j-k}$, temos que:

$$
\begin{aligned}
& \left|\left(\tau_{0}^{p_{k+1}}-\tau_{0}^{p_{k}}\right)-\tau_{0}^{p_{k+1}-p_{k}}\right|=\left|\left(\tau_{0}^{p_{k+1}}-\tau_{0}^{p_{k}}\right)-\tau_{-p_{k}+p_{k}}^{p_{k+1}-p_{k}}\right|=\mid\left(\tau_{0}^{p_{k+1}}-\tau_{0}^{p_{k}}\right)-\left(\tau_{-p_{k}}^{p_{k+1}}-\tau_{-p_{k}}^{p_{k}}\right) \leq \leq \\
& \leq\left|\left(\tau_{-p_{k}}^{p_{k}}-\tau_{0}^{p_{k}}\right)+\right|\left(\tau_{-p_{k}}^{p_{k}}-\tau_{0}^{p_{k+1}}\right) .
\end{aligned}
$$

Sabemos que $p_{k}$ e $p_{k+1}$ estão em $\mathrm{P}_{\varepsilon}$. Assim, ambos são $2 \varepsilon$-quase-períodos de todos os $\tau_{i}^{j}$. Assim, para $j=p_{k}$ e para $j=p_{k+1}$, temos que $\left|\tau_{-p_{k}}^{p_{k}}-\tau_{0}^{p_{k}}\right|<2 \varepsilon$ e $\left|\tau_{-p_{k}}^{p_{k+1}}-\tau_{0}^{p_{k+1}}\right|<2 \varepsilon$. Lembremos que se $p$ é um $\varepsilon$-quase-período, então - $p$ é também um $\varepsilon$-quase-período. Assim, $\left|\left(\tau_{0}^{p_{k+1}}-\tau_{0}^{p_{k}}\right)-\tau_{0}^{p_{k+1}-p_{k}}\right|$ é limitada. 
Assim, assuma $p_{k+1}-p_{k}$ limitada. Então, $p_{k+1}-p_{k}$ toma valores em um conjunto finito. Assim, há somente finitos valores para $\tau_{0}^{p_{k+1}-p_{k}}$, o que faz com que $\tau_{0}^{p_{k+1}}-\tau_{0}^{p_{k}}$ seja limitada. Inversamente, admita que $\tau_{0}^{p_{k+1}}-\tau_{0}^{p_{k}}$ é limitado. Então, $\tau_{0}^{p_{k+1}-p_{k}}$ é limitado, isto é, $\tau_{p_{k+1}-p_{k}}-\tau_{0}$ é limitado já que $\tau_{p_{k+1}-p_{k}}$ é limitado. Como $\tau_{i} \rightarrow \infty$ quando $i \rightarrow \infty$, não podemos ter $p_{k+1}-p_{k}$ ilimitado.

Lema 5 - As seguintes afirmações são equivalentes:

a) As seqüências $\left\{\tau_{i}^{j}\right\}, i, j \in Z$, são equipotencialmente quase-periódicas.

b) O conjunto $\mathrm{P}$ é relativamente denso para algum $\varepsilon>0$.

c) O conjunto $\Gamma_{\varepsilon}$ é relativamente denso para algum $\varepsilon>0$.

Demonstração:

Que b) implica c) e que c) implica b), basta utilizar o lema 4. Vamos mostrar que a) implica b). Devemos mostrar que dado $\varepsilon>0, \mathrm{P}_{\varepsilon}$ é relativamente denso, isto é, existe $L>0$ tal que para qualquer $x \in \Re$, tenhamos $[x, x+L] \cap \mathrm{P}_{\varepsilon} \neq \varnothing$.

Sabemos que existe um conjunto relativamente denso de $\varepsilon$-quase-períodos para todo $\tau_{i}^{j}$. Assim, existe $l>0$ tal que para qualquer $z \in \Re$, temos que $[z, z+L]$ contém um $\varepsilon$-quase-período $p$ para todo $\tau_{i}^{j}$.

Agora, se tomarmos $L=l, z=x$, temos que $p \in[x, x+L]$. Além disso, pelo Lema 3, sabemos que $p \in \mathrm{P}_{\varepsilon}$. Logo, $[x, x+L] \cap \mathrm{P}_{\varepsilon} \neq \varnothing$, o que prova que $\mathrm{P}_{\varepsilon}$ é relativamente denso.

Vamos agora mostrar que b) implica a). Devemos mostrar que dado $\varepsilon>0$, existe $L>0$ tal que para qualquer $x \in \mathfrak{R}$, o intervalo $[x, x+L]$ contém um $\varepsilon$-quase-período $p$. Sabemos que $\mathrm{P}_{\varepsilon / 2}$ é relativamente denso e assim, existe $l>0$ tal que para qualquer $x \in \mathfrak{R}$, é possível encontrar $p \in[x, x+l] \cap \mathrm{P}_{\varepsilon / 2}$.

Assim, seja $L=l$. Dado $x$, seja $p \in[x, x+l] \cap \mathrm{P}_{\varepsilon / 2}$. Pelo Lema $3, p$ é um $\varepsilon$-quaseperíodo para todo $\tau_{i}^{j}$. 
Lema 6 - Se a família de seqüências $\left\{\tau_{i}^{j}\right\}$ é equipotencialmente quase-periódica, então para algum $i>0$ existe um número natural $d$ tal que em qualquer intervalo de comprimento $l>0$ não há mais que $d$ termos na seqüência $\left\{\tau_{i}\right\}$.

\section{Demonstração:}

Escolha $\varepsilon=1$. Como $\tau \in \Gamma_{\varepsilon}$ é relativamente denso pelo lema 5, então existe $L>0$ tal que $[x, x+L] \cap \Gamma_{\varepsilon} \neq \varnothing$ para qualquer $x \in \Re$. Então, para qualquer $l>0$, podemos escolher $x$ tal que $1+l<x$. Então, existe um número $r \in[x, x+L] \cap \Gamma_{\varepsilon}$ tal que $l<r-1$. Como $l>0$, temos que $r>1$.

Além disso, existe $k \in Z$ tal que para qualquer $i \in Z$, temos $\left|\tau_{i}^{k}-r\right|<1$. Em particular, se $i=0$, temos que $\left|\tau_{0}^{k}-r\right|<1$. Como $r>1$ e $\left|\tau_{0}^{k}-r\right|<1$, temos que $1<r=r-\tau_{0}^{k}+\tau_{0}^{k}<1+\tau_{0}^{k}$. Logo, $\tau_{0}^{k}>0$, isto é $0<\tau_{0}^{k}<\tau_{k}-\tau_{0} \Rightarrow \tau_{k}>\tau_{0}$ e desse modo, $k \geq 1$.

Escolha $d=k$ e sejam $(a, a+l)$ um intervalo e $\tau_{i_{0}}$ o menor termo da seqüência $\left\{\tau_{i}\right\}$ que pertence a este intervalo. Como $\left|\tau_{0}^{k}-r\right|<1$, temos $-1<\tau_{i_{0}}^{k}-r=\tau_{i_{0}+k}-\tau_{i_{0}}-r$, isto é, $r-1+\tau_{i_{0}}<\tau_{i_{0}+k}$. Mas, $l<r-1$. Então, $l+\tau_{i_{0}}<\tau_{i_{0}+k}$. Ainda, temos que $a<\tau_{i_{0}}$, pois $\tau_{i_{0}}$ é o menor elemento da seqüência $\left\{\tau_{i}\right\}$ que pertence ao intervalo $(a, a+l)$. Logo, $a+l<\tau_{i_{0}+k}$, isto é, $\tau_{i_{0}+k}$ já não pertence ao intervalo $(a, a+l)$.

Para o lema a seguir considere o sistema de equações diferenciais dado por $\frac{d x}{d t}=A(t) x$, onde $A(t)$ é uma matriz quadrada $n \times n$, contínua com relação a $t$.

Lema 7 - Seja $U(t, s)$ a matriz fundamental de soluções do sistema $\frac{d x}{d t}=A(t) x, A(t)$ quase-periódica, $f(t)$ é função quase-periódica, a seqüência de vetores $\left\{I_{i}\right\}$ e as matrizes $\left\{B_{i}\right\}$ são quase-periódicas, a seqüência de instantes $\left\{\tau_{i}\right\}$ é tal que as seqüências $\left\{\tau_{i}^{j}\right\}$, $i, j \in Z$, são positivas e equipotencialmente quase-periódicas. Então, para qualquer $\varepsilon>0$ e qualquer $\theta>0$, existem um número $v, 0<v<\varepsilon$, um conjunto de números 
reais relativamente denso $\Gamma$ e um conjunto de números inteiros $Q$ que satisfazem às seguintes relações:

1) $\|U(t+\tau, s+\tau)-U(t, s)\|<\mathcal{E} \quad 0 \leq t-s \leq \theta$ e $\tau \in \Gamma$.

2) $\|f(t+\tau)-f(t)\|<\varepsilon \quad t \in \mathfrak{R},\left|t-\tau_{i}\right|>\varepsilon, i=0, \pm 1, \ldots$,

3) $\left\|I_{k+q}-I_{q}\right\|<\varepsilon, \quad q \in Q, \quad k=0, \pm 1, \ldots$,

4) $\left\|B_{k+q}-B_{q}\right\|<\mathcal{E}, \quad q \in Q, \quad k=0, \pm 1, \ldots$

5) $\left\|\tau_{k}^{q}-\tau\right\|<\varepsilon, \quad q \in Q, \quad k=0, \pm 1, \ldots$.

Demonstração:

Pode ser encontrada em [1], lema 35.

Lema 8 - Sejam $A(t)$ a matriz quase-periódica do sistema $\frac{d x}{d t}=A(t) x,\left\{B_{i}\right\}$ matrizes quase-periódicas, e $\left\{\tau_{i}^{j}\right\}, i, j \in Z$ seqüências equipotencialmente quase-periódicas. Se a matriz $X(t, s)$, matriciante do sistema (3), satisfaz a desiguladade $\|X(t, s)\| \leq C e^{-\alpha(t-s)}, \quad t \geq s$, onde $C$ e $\alpha$ são números reais positivos, então a diagonal da matriz $\mathrm{X}(\mathrm{t}, \mathrm{s})$ é quase-periódica, isto é, para alguns $\varepsilon>0, t, s \in \Re, \quad|t-\tau+i|>\varepsilon, \quad\left|s-\tau_{i}\right|>\varepsilon, i=0, \pm 1, \ldots$, existe um conjunto relativamente denso de quase-períodos $\Gamma$ tal que para qualquer $\tau \in \Gamma$ temos que:

$$
\|X(t+\tau, s+\tau)-X(t, s)\|<\varepsilon C e^{-\frac{\alpha}{2}(t-s)} \text {, onde } C \text { é uma constante positiva. }
$$

Demonstração:

Temos que:

$\frac{\partial X}{\partial t}=A(t+r) X(t+r, s+r), t \neq \tau_{i}^{\prime}$

$\frac{\partial X}{\partial t}=A(t+r) X(t+r, s+r)+A(t) X(t+r, s+r)-A(t) X(t+r, s+r), t \neq \tau_{i}^{\prime}$ 
Logo:

$\frac{\partial X}{\partial t}=A(t) X(t+r, s+r)+(A(t+r)-A(t)) X(t+r, s+r), t \neq \tau_{i}^{\prime}$. Temos também que $\left.\Delta X(t+\tau, s+\tau)\right|_{t=\tau^{\prime}}=B_{i} X\left(\tau_{i}^{\prime}+r, s+\tau\right)+\left(B_{i+q}-B_{i}\right) X\left(\tau_{i}+r, s+\tau\right), \quad$ onde $\quad \tau_{i}^{\prime}=\tau_{i}-\tau$.

Tomemos os números $\tau$ e $q$ definidos de acordo com as condições do lema 7 .

Se escolhermos $x_{0}$ como sendo um dos vetores da base canônica de $\Re^{n}, e_{1}, e_{2}, \ldots, e_{n}$, e considerarmos a matriz $n x n$ em cada coluna é dada por $X\left(t, e_{i}\right), i=1, \ldots, n$, isto é, a matriz $\left[X\left(t, e_{1}\right) \ldots X\left(t, e_{n}\right)\right]_{n x n}$, temos de acordo com o corolário 1 do teorema 4 que:

$\left[X\left(t, e_{1}\right) \ldots X\left(t, e_{n}\right)\right]=X\left(t, t_{0}\right)+\left[\int_{t_{0}}^{t} X(t, \tau) f_{1}(\tau) \mathrm{d} \tau \ldots . \int_{t_{0}}^{t} X(t, \tau) f_{n}(\tau) \mathrm{d} \tau\right]+\left[\sum_{t_{0}<\tau_{i}<t} X\left(t, \tau_{i}+\right) a_{1 i} \ldots \sum_{t_{n}<\tau_{i}<t} X\left(t, \tau_{i}+\right) a_{n i}\right]$

Nesta expressão, $x\left(t, x_{0}\right)$ é solução de $\frac{d x}{d t}=A(t) x+f_{i}(t)$, onde $f_{i}$ é a i-ésima coluna da matriz $[A(t+\tau)-A(t)] X(t+r, s+r)$.

Em outras palavras, considere $n$ sistemas da forma $\frac{d X_{i}}{d t}=A(t) X_{i}+[A(t+\tau)-A(t)] X(t+r, s+r) e_{i}$ com $X_{i}\left(t, e_{i}\right)$ no instante $s$ sendo $X_{i}\left(s, e_{i}\right)=e_{i}$. Então:

$X_{i}\left(t, e_{i}\right)=X(t, s) e_{i}+\int_{s}^{t} X(t, \tau)[A(t+\tau)-A(t)] X(\tau+r, s+r) e_{i} \mathrm{~d} \tau+\sum_{s<\tau_{i}^{<<t}} X\left(t, \tau_{i}\right)\left(B_{i+q}-B_{i}\right) X\left(\tau_{i}+r, s+r\right) e_{i}$

Se considerarmos a matriz $\left[X_{1}\left(t, e_{1}\right) \ldots . X_{n}\left(t, e_{n}\right)\right]_{n x n}$, temos que:

$X(t+\tau, s+\tau)=X(t, \tau)+\int_{s}^{t} X(t, u)[A(u+\tau)-A(u)] X(u+\tau, s+\tau) d u+$

$+\sum_{v \leq \tau_{i}^{\prime} \leq t} X\left(t, \tau_{i}^{\prime}\right)\left(B_{i+q}-B_{i}\right) X\left(\tau_{i}^{\prime}+\tau, s+\tau\right)$

pois $X(t+\tau, s+\tau)$ é solução também e assim deve valer a igualdade.

Pelo lema 7, temos que $\left|t-\tau_{i}^{\prime}\right|>\varepsilon$ implica que $\tau_{i+q}^{\prime}<t+\tau<\tau_{i+q+1}^{\prime}$. Desse modo, temos que: 


$$
\begin{aligned}
& \|X(t+\tau, s+\tau)-X(t, s)\| \leq \int_{s}^{t}\|X(t, u)\| A(u+\tau)-A(u)\|X(u+\tau, s+\tau)\| d u+ \\
& +\sum_{s \leq t_{i} \leq t}\left\|X\left(t, \tau_{i}^{\prime}\right)\right\| B_{i+q}-B_{i}\left\|X\left(\tau_{i}^{\prime}+\tau, s+\tau\right)\right\| \leq \\
& \leq \int_{s}^{t} \varepsilon C_{1} e^{-\alpha(t-s)} d u+\sum_{s<\tau_{i}<t} \varepsilon C_{2} e^{-\alpha(t-s)}=\frac{\varepsilon C_{1}}{\alpha} e^{-\alpha(t-s)}(t-s)+i(s, t) \varepsilon C_{2} e^{-\alpha(t-s)},
\end{aligned}
$$

Onde o número $i(s, t)$ corresponde ao número de pontos $\tau_{t}^{\prime}$ no intervalo aberto $(s, t) \mathrm{e}$ $C_{1}, C_{2}$ são constantes. Seja $N$ o número definido como no lema 6 . Então,

$$
\|X(t+\tau, s+\tau)-X(t, s)\| \leq \varepsilon\left(\frac{2 C_{1}}{\alpha^{2}}+\frac{2 N C_{2}}{\alpha}\right) e^{-\frac{\alpha}{2}(t-s)}=\varepsilon C e^{-\frac{\alpha}{2}(t-s)}
$$

Considere agora o sistema diferencial com impulso:

$$
\begin{aligned}
& \frac{d x}{d t}=A(t) x+f(t), \quad t \neq \tau_{i}, \\
& \left.\Delta x\right|_{t=\tau_{i}}=B_{i} x+I_{i}
\end{aligned}
$$

Onde as matrizes $A(t), B_{i}, i=0, \pm 1, \pm 2, \ldots$, as seqüências $\left\{\tau_{i}\right\},\left\{I_{i}\right\}$ e a função $f(t)$ satisfazem as condições dos lemas 7 e 8 . Vamos usar a seguinte notação:

$$
\gamma=\sup \Lambda(t), \quad \alpha^{2}=\sup _{t} \Lambda_{i}^{2}
$$

onde $\Lambda(t)$ é o maior autovalor da matriz $\frac{1}{2}\left(A(t)+A^{c}(t)\right)$ e $\Lambda_{i}$ é o maior autovalor da matriz $\left(I d+B_{i}\right)^{C}\left(i d+B_{i}\right)$, e $C$ é o operador conjugação, isto é , se $a_{i j}=A$, $a_{i j}=b_{i j}+c_{i j} i$, então $a_{i j}{ }^{c}=b_{i j}-c_{i j} i$. Pelo lema 2, existe e é finito o limite:

$$
\lim _{t \rightarrow \infty} \frac{i\left(t_{0}, t_{0}+t\right)}{t}=p
$$


que é uniforme para $t_{0} \in \Re$. Pelo teorema 5 , temos que $\|X(t, s)\| \leq K e^{(\varepsilon+\gamma+p \ln \alpha)(t-s)}$, onde $\varepsilon>0$ é arbitrário, $K=K(\varepsilon) \geq 1$. Vamos denotar por $\beta(\varepsilon)=\varepsilon+\gamma+p \ln \alpha$.

Teorema 7 - Se o sistema (8) satisfaz as condições acima listadas e $\gamma+p \ln \alpha<0$, então tal sistema admite uma única solução quase-periódica e esta solução é assimptoticamente estável.

Demonstração:

Uma solução quase-periódica do sistema (8) é dada pela expressão:

$$
x_{0}(t)=\int_{-\infty}^{t} X(t, s) f(s) d s+\sum_{t_{i}<t} X\left(t, t_{i}\right) I_{i} .
$$

Para isso, basta substituir essa expressão no sistema (8).

Tome agora um $\varepsilon$-período como definido nos lemas 7 e 8 , e usando o lema 6 , encontramos:

$$
\begin{aligned}
& \left\|x_{0}(t+\tau)-x_{0}(t)\right\| \leq \int_{-\infty}^{t}\|X(t+\tau, s+\tau) f(s+\tau)-X(t, s) f(s)\| d s+ \\
& +\sum_{\tau_{i}<i}\left\|X\left(t+\tau, \tau_{i+d}\right) I_{i+d}-X\left(t, \tau_{i}\right) I_{i}\right\|<\Gamma_{1}(\varepsilon) \mathcal{E},
\end{aligned}
$$

onde $\Gamma_{1}(\varepsilon)$ é uma função positiva e limitada de $\varepsilon$. Isso mostra que a solução é quaseperiódica.

Como as soluções de (8) podem ser representadas por

$$
x(t)=X\left(t, t_{0}\right) x_{0}+\int_{t_{0}}^{t} X(t, s) f(s) d s+\sum_{t_{0} \leq \tau_{i}<t} X\left(t, \tau_{i}\right) I_{i},
$$

Então para duas soluções quaisquer de (8), $\varphi(t)$ e $\psi(t)$, temos que: 
$\|\varphi(t)-\psi(t)\| \leq K e^{\beta\left(1-t_{0}\right)}\left\|\varphi\left(t_{0}\right)-\psi\left(t_{0}\right)\right\|$.

Segue que a solução de (8) é assimptoticamente estável e única.

O teorema 7 e o teorema 1, apresentado na seção anterior, são os teoremas mais importantes para nossa aplicação. Vamos agora retomar o modelo de PAS e aplicar nele a teoria de EDIs até aqui desenvolvida. 


\section{3 - Aplicações das EDIs em um modelo de PAS}

Vimos, apesar de algumas simplificações, que as variações na PAS satisfazem às seguintes equações:

$$
\frac{d P_{s a}(t)}{d t}=-\alpha P_{s a}(t), \alpha=-\frac{1}{C_{s a} R_{S}}, \text { se } t=t_{i},
$$

onde $t_{i+1}-t_{i}=T, i \in Z, T \in \mathfrak{R}^{+}, \mathrm{e}$

$$
P\left(t_{i}+\right)=P\left(t_{i}-\right)+I
$$

onde $I>0$ é fixado, $P\left(t_{i}+\right)$ e $P\left(t_{i}-\right)$ são os valores da PAS depois e antes da contração cardíaca no instante $t_{i}$. Essas equações podem ser reescritas na forma:

(3) $\left\{\begin{array}{c}\frac{d P_{s a}(t)}{d t}=-\alpha P_{s a}(t) \quad t \neq t_{i} \\ \left.\Delta P_{s a}\right|_{t=t_{i}}=I_{i}\end{array}\right.$,

onde $\left.\Delta P_{s a}\right|_{t=t_{i}}=P_{s a}\left(t_{i}+\right)-P_{s a}\left(t_{i}-\right), I_{i+1}=I_{i}, \quad i \in Z$. Além do sistema (3), vamos considerar também o seguinte sistema:

(4) $\left\{\begin{array}{c}\frac{d P_{s a}(t)}{d t}=-\alpha P_{s a}(t)+Q(t) \quad t \neq t_{i} \\ \left.\Delta P_{s a}\right|_{t=t_{i}}=I_{i}\end{array}\right.$,

onde $Q(t) \in P C$. O sistema (4) é chamado de sistema $Q$-perturbado.

Da mesma forma que no modelo dinâmico construído no capítulo 1 , o volume bombeado pelo coração é dado por $\Delta V=C_{s a} \Delta P_{s a}$, onde $\left.\Delta P_{s a}\right|_{t=t_{i}}=P_{s a}\left(t_{i}+\right)-P_{s a}\left(t_{i}-\right)$. Já o fluxo cardíaco $(Q)$ é simplesmente a razão entre volume bombeado pelo coração e 
o período $\left(T_{i}\right)$ entre duas sístoles. Como freqüência $(F)$ é o inverso do período, temos que $Q_{i}=\Delta V_{i} F_{i}$.

Os sistemas (3) e (4) são sistemas de equações diferenciais com impulso. No que segue, vamos verificar condições acerca da existência de soluções dos sistemas (3) e (4).

a) Existência de uma solução positiva, assimptoticamente estável e T-periódica do sistema (3).

Sejam $t_{i}=i T, i \in Z$ os momentos de salto das soluções do sistema (3), $I_{i}=I d$, $i \in Z$ e $P(t), P(0)=P_{0}>0$ uma solução de (3). Pelo corolário 1 , sabemos que a solução é dada por:

$$
P(t)=e^{-\alpha t}\left[P_{0}+\sum_{0 \leq i_{i}<t} e^{\alpha t_{i}} I d\right]
$$

Denote por $P^{0}=\left(e^{\alpha T}-1\right)^{-1}$ e seja $P_{0}(t), P_{0}(0)=P^{0}$ uma solução de (3). Então,

$$
P_{0}(t+T)=e^{-\alpha(t+T)}\left[P^{0}+\sum_{0 \leq t_{i}<t+T} e^{\alpha t_{i}}\right]=e^{-\alpha t} e^{-\alpha T}\left[\frac{1}{e^{\alpha T}-1}+\sum_{0 \leq t_{t}<t+T} e^{\alpha\left(t_{i}-T\right)}\right]
$$

Suponha que no intervalo $[0, t[$ existam $m$ saltos. Então, como os saltos ocorrem com período $\mathrm{T}$, segue que no intervalo $[0, t+T$ [ existirão $m+1$ saltos. Assim,

$$
\begin{aligned}
& P_{0}(t+T)=e^{-\alpha t} e^{-\alpha T}\left[\frac{1}{e^{\alpha T}-1}+e^{0}+\ldots+e^{\alpha d_{m}}+e^{\alpha d_{m+1}}\right]=e^{-\alpha t} e^{-\alpha T}\left[\frac{1}{e^{\alpha T}-1}+1+\ldots+e^{\alpha m T}+e^{\alpha(m+1) T}\right]= \\
& =e^{-\alpha t}\left[\frac{e^{-\alpha T}}{e^{\alpha T}-1}+e^{-\alpha T}+1+\ldots+e^{\alpha(m-1) T}+e^{\alpha m T}\right]=e^{-\alpha t}\left[\frac{1-e^{-\alpha T}+e^{-\alpha T}}{e^{\alpha T}-1}+\sum_{0 \leq t_{i}<t} e^{\alpha t_{i}}\right]= \\
& e^{-\alpha t}\left[\frac{1}{e^{\alpha T}-1}+\sum_{0 \leq t_{i}<t} e^{\alpha t_{i}}\right]=e^{-\alpha t}\left[P^{0}+\sum_{0 \leq t_{i}<t} e^{\alpha t_{i}}\right]=P_{0}(t+T)
\end{aligned}
$$


Assim $P_{0}(t)$ é solução T-periódica, pois $P_{0}(t+T)=P_{0}(t)$ para todo $t$.

Seja $P(t)$ uma solução arbitrária do sistema (3), $P(0)=P_{0}>0$. Então,

$$
P(t)-P_{0}(t)=e^{-\alpha t}\left(P_{0}+\sum_{0 \leq t_{i}<t} e^{\alpha t_{i}}\right)-e^{-\alpha t}\left(P^{0}+\sum_{0 \leq t_{i}<t} e^{\alpha t_{i}}\right)=e^{-\alpha t}\left(P_{0}-P^{0}\right) .
$$

Então, $\quad P(t)-P_{0}(t) \rightarrow 0$ quando $t \rightarrow \infty$. Assim, $P_{0}(t)$ é uma solução assimptoticamente estável e T-periódica do sistema (3).

b) Existência de uma solução positiva, assimptoticamente estável e T-periódica do sistema (4) quando $Q(t)$ é uma função positiva T-periódica.

Assuma que $Q(t)$ é T-periódica. Seja $P(t)$ uma solução do sistema (4), $P(0)=P_{0}$. Então, pelo corolário 1, temos que:

$$
P(t)=e^{-\alpha t}\left[P_{0}+\int_{0}^{t} e^{\alpha s} Q(s) d s+\sum_{0 \leq t_{i}<t} e^{\alpha t_{i}}\right]
$$

Denote por $P^{0}=\left(e^{\alpha T}-1\right)^{-1}\left(\int_{0}^{T} e^{\alpha s} Q(s) d s+I d\right)$ e seja $P_{0}(t), P_{0}(0)=P^{0}$ uma solução de (4). Vamos mostrar que $P_{0}(t)$ é periódica, isto é, que $P_{0}(t+T)=P_{0}(t)$.

$$
\begin{aligned}
& P(t+T)=e^{-\alpha(t+T)}\left[P^{0}+\int_{0}^{t+T} e^{\alpha s} Q(s) d s+\sum_{0 \leq t_{i}<t+T} e^{\alpha t_{i}}\right]= \\
& e^{-\alpha t} e^{-\alpha T}\left[\frac{1}{e^{\alpha T}-1}\left(\int_{0}^{T} e^{\alpha s} Q(s) d s+1\right)+\int_{0}^{t+T} e^{\alpha s} Q(s) d s+1+\ldots+e^{\alpha(m+1) T}\right]= \\
& e^{-\alpha t}\left[\frac{e^{-\alpha T}}{e^{\alpha T}-1}\left(\int_{0}^{T} e^{\alpha s} Q(s) d s+1\right)+e^{-\alpha T} \int_{0}^{t+T} e^{\alpha s} Q(s) d s+e^{-\alpha T}+\sum_{0 \leq t_{i}<t} e^{\alpha t_{i}}\right] .
\end{aligned}
$$


Mas, $\int_{0}^{t+T} e^{\alpha s} Q(s) d s=\int_{0}^{T} e^{\alpha s} Q(s) d s+\int_{T}^{t+T} e^{\alpha s} Q(s) d s$. Fazendo uma mudança de variável $(u=s-T)$ no segundo termo do lado direito da equação, teremos que:

$$
\int_{0}^{t+T} e^{\alpha s} Q(s) d s=\int_{0}^{T} e^{\alpha s} Q(s) d s+e^{\alpha T} \int_{0}^{t} e^{\alpha s} Q(s) d s
$$

Temos então:

$$
\begin{aligned}
& P_{0}(t+T)=e^{-\alpha s}\left[\frac{e^{-\alpha T}}{e^{\alpha T}-1}\left(\int_{0}^{T} e^{\alpha s} Q(s) d s+1\right)+e^{-\alpha T}\left(\int_{0}^{T} e^{\alpha s} Q(s) d s+e^{\alpha T} \int_{0}^{t} e^{\alpha s} Q(s) d s\right)+e^{-\alpha T}+\sum_{0 \leq t_{i}<t} e^{\alpha t_{i}}\right]= \\
& =e^{-\alpha t}\left[\frac{e^{-\alpha T}}{e^{\alpha T}-1}\left(\int_{0}^{T} e^{\alpha s} Q(s) d s+1\right)+e^{-\alpha T} \int_{0}^{T} e^{\alpha s} Q(s) d s+\int_{0}^{t} e^{\alpha s} Q(s) d s+e^{-\alpha T}+\sum_{0 \leq t_{i}<t} e^{\alpha t_{i}}\right]= \\
& =e^{-\alpha t}\left[\frac{1}{e^{\alpha T}-1}\left(e^{-\alpha T} \int_{0}^{T} e^{\alpha s} Q(s) d s+e^{-\alpha T}+\int_{0}^{T} e^{\alpha s} Q(s) d s-e^{-\alpha T} \int_{0}^{T} e^{\alpha s} Q(s) d s+1+e^{-\alpha T}\right)+\int_{0}^{t} e^{\alpha s} Q(s) d s+\sum_{0 \leq t_{i}<t} e^{\alpha t_{i}}\right]= \\
& =e^{-\alpha t}\left[\frac{1}{e^{\alpha T}-1}\left(\int_{0}^{T} e^{\alpha s} Q(s) d s+1\right)+\int_{0}^{t} e^{\alpha s} Q(s) d s+\sum_{0 \leq t_{i}<t} e^{\alpha t_{i}}\right]=P_{0}(t)
\end{aligned}
$$

Assim, temos que $P_{0}(t)$ é T-periódica. Além disso, como $Q(t)>0$, então a solução $P_{0}(t)$ é positiva para todo $t$.

Seja $P(t)$ uma solução arbitrária do sistema (4). Então, da mesma forma que em a), temos que

$$
P(t)-P_{0}(t)=e^{-\alpha t}\left(P_{0}-P^{0}\right) .
$$

Assim, $P_{0}(t)$ é uma solução assimptoticamente estável do sistema $Q$-perturbado.

c) Existência de uma solução assimptoticamente estável e $\mathcal{E}$-oscilatória do sistema (4), $\operatorname{com} \varepsilon$ arbitrariamente pequeno, quando $Q(t)$ não é periódica, mas é pequena na norma do sup e o sistema não perturbado é periódico. 
Vamos assumir que o sistema (3), associado ao sistema (4), satisfaz as condições do problema a) e a função $Q(t)$ não é T-periódica, mas existe um número $\delta>0$ tal que $|Q(t)| \leq \delta, t \geq 0$. Devemos mostrar que para um dado $\varepsilon>0$, existe uma $\varepsilon$ oscilação de (4), se $\delta$ é suficientemente pequeno.

De fato, assuma que $\delta<\alpha \varepsilon$. Seja a função $P_{0}(t)$ definida pela fórmula:

$$
P_{0}(t)=e^{-\alpha t}\left[P^{0}+\sum_{0 \leq t_{i}<t} e^{c s_{i}}\right]
$$

onde $P^{0}=\left(e^{\alpha T}-1\right)^{-1}$. Já foi provado que $P_{0}(t)$ é uma função periódica. Seja $\tilde{P}(t), \tilde{P}(0)=P^{0} \quad$ uma solução do sistema (4). Então, usando
$\tilde{P}(t)=e^{-\alpha t}\left[P_{0}+\int_{0}^{t} e^{\alpha s} Q(s) d s+\sum_{0 \leq t_{i}<t} e^{\alpha t_{i}}\right]$ obtemos: $\tilde{P}(t)-P_{0}(t)=\int_{0}^{t} e^{-\alpha(t-s)} Q(s) d s$,

assim, $\left|\tilde{P}(t)-P_{0}(t)\right| \leq \varepsilon$. Logo, a solução $\tilde{P}(t)$ é $\varepsilon$-oscilatória.

Denote por $\beta=\min _{0 \leq \leq \leq T} P_{0}(t)$. Assuma que $\varepsilon$ é tal que $\varepsilon<\beta$. Temos então que $\tilde{P}(t)>0$ para todo $t$.

Seja $P(t)$ solução de do sistema (4) definida por $P(t)=e^{-\alpha t}\left[P_{0}+\int_{0}^{t} e^{\alpha s} Q(s) d s+\sum_{0 \leq t_{i}<t} e^{\alpha t_{i}}\right]$. Então $|P(t)-\tilde{P}(t)|=e^{-\alpha t}\left(P^{0}-P_{0}\right)$. Assim, $\tilde{P}(t)$ é assimptoticamente estável.

d) Existência de uma solução quase-periódica e assimptoticamente estável do sistema (3) tal que todos seus elementos são quase-periódicos. 
Consideraremos o problema de existência de soluções contínuas por partes e quaseperiódicas do sistema (3), onde $I_{i}$ é uma seqüência quase-periódica e as seqüências $t_{i}^{j}, j \in Z$, derivadas de $t_{i}, i \in Z$, são equipotencialmente quase-periódicas.

Usando o teorema 7, temos que a função

$$
P^{0}(t)=\sum_{t_{i}<t} e^{-\alpha\left(t-t_{i}\right)} I_{i}
$$

é solução contínua por partes, quase-periódica e assimptoticamente estável do sistema (3). Além disso, tal solução é positiva.

e) Existência de uma solução assimptoticamente estável e quase-periódica do sistema $Q$-perturbardo, quando $Q(t)$ é função contínua por partes e quaseperiódica.

Assumimos aqui que todos os elementos, exceto $Q(t)$, são os mesmos do problema d) e que $Q(t)$ é uma função contínua por partes e quase-periódica. Novamente usando o teorema 7 temos que:

$$
P(t)=\int_{-\infty}^{t} e^{-\alpha(t-s)} Q(s) d s+\sum_{t_{i}<t} e^{\alpha\left(t-t_{i}\right)} I_{i}
$$

é solução assimptoticamente estável e quase-periódica do sistema $Q$-perturbardo.

f) Existência de uma solução estável e $\varepsilon$-oscilatória, com $\varepsilon>0$ arbitrariamente pequeno, do sistema (4) quando $Q(t)$ é positiva e pequena na norma do sup e o sistema (3), associado ao sistema (4), é quase-periódico.

Vamos supor que a equação $Q(t)$ satisfaz $|Q(t)|<\delta, t \in \Re, \delta<\varepsilon|\alpha|$. Então $P(t)=\int_{-\infty}^{t} e^{-\alpha(t-s)} Q(s) d s+\sum_{t_{i}<t} e^{\alpha\left(t-t_{i}\right)} I_{i}$ é solução do sistema (4) pelo Teorema 7 e tanto a integral como a série são convergentes. 
De fato, $\int_{-\infty}^{t} e^{-\alpha(t-s)} Q(s) d s=e^{-\alpha t} \int_{-\infty}^{t} e^{\alpha s} Q(s) d s$

Basta analisarmos a convergência de $\int^{t} e^{\alpha s} Q(s) d s$. Como $Q(t)$ é positiva, temos que: $0 \leq\left|\int_{-\infty}^{t} e^{\alpha s} Q(s) d s\right| \leq \int_{-\infty}^{t} e^{|\alpha| s} \delta d s=\delta \lim _{u \rightarrow-\infty} \int_{u}^{t} e^{|\alpha| s} d s=\left.\delta \lim _{u \rightarrow-\infty} \frac{e^{|\alpha| s}}{|\alpha|}\right|_{u} ^{t}=\delta \lim _{u \rightarrow-\infty}\left(\frac{e^{|\alpha| t}}{|\alpha|}-\frac{e^{|\alpha| u}}{|\alpha|}\right)=$ $=\delta \frac{e^{|\alpha| t}}{|\alpha|}$.

Segue do critério da comparação que a integral em questão é convergente.

Vamos agora verificar a convergência da série. Para isso, denotamos $s_{i}=\sum_{t_{i}<t} e^{\alpha\left(t-t_{i}\right)} I_{i}$. Como $I_{i}$ é seqüência quase periódica, segue que $I_{i}$ é limitada, isto é, existe $M>0$ tal que $I_{i}<M$. Assim, $s_{i}=\sum_{t_{i}<t} e^{\alpha\left(t-t_{i}\right)} I_{i} \leq M \sum_{t_{i}<t} e^{\alpha\left(t-t_{i}\right)}$ e basta analisarmos a convergência de $S_{i}=\sum_{t_{i}<t} e^{\alpha\left(t-t_{i}\right)}=\sum_{t_{i}<t} x_{i}$. Aplicando o teste da razão, temos que $\frac{x_{n}}{x_{n+1}}=\frac{e^{\alpha(t-n T)}}{e^{\alpha(t-(n+1) T)}}=e^{\alpha T}<1$, pois $\alpha<0$. Desse modo, a série em questão é convergente.

Se subtrairmos da solução $P^{0}(t)=\sum_{t_{i}<t} e^{-\alpha\left(t-t_{i}\right)} I_{i}$, temos que:

$$
\begin{aligned}
& \left|P(t)-P^{0}(t)\right|=\left|\int_{-\infty}^{t} e^{-\alpha(t-s)} Q(s) d s\right|<\delta\left|\int_{-\infty}^{t} e^{-\alpha(t-s)} d s\right| \leq \delta\left|e^{-|\alpha| t} \int_{-\infty}^{t} e^{|\alpha| \cdot s} d s\right|=\delta \mid e^{-|\alpha| t}\left(\lim _{u \rightarrow-\infty}\left(\frac{e^{|\alpha| t}}{|\alpha|}-\frac{e^{|\alpha| u}}{|\alpha|}\right)\right)= \\
& \delta\left|e^{-|\alpha| t}\left(\frac{e^{|\alpha| t}}{|\alpha|}\right)\right|=\frac{\delta}{|\alpha|}<\varepsilon
\end{aligned}
$$

Assim, $P(t)$ é solução estável e $\varepsilon$-oscilatória do sistema (4). 
Até aqui estudamos o caso em que os momentos de salto ocorriam em momentos determinados. Vamos agora verificar o caso em que as descontinuidades ocorrem quando a PAS atinge um valor mínimo especial $\bar{P} \in \mathfrak{R}, \bar{P}>0$. Como vimos, o mecanismo de controle do fluxo cardíaco é controlado pelos baroreceptores. Os baroreceptores estão localizados nas paredes das grandes artérias do sistema circulatório, no arco aórtico e no sino da carótida. A queda da pressão faz com que esse mecanismo passe a atuar fazendo aumentar o fluxo cardíaco. O modelo que representa essa situação será descrito por:

(5) $\left\{\begin{array}{c}\frac{d P(t)}{d t}=-\alpha P(t) \quad P \neq \bar{P} \\ \left.\Delta P\right|_{P=\bar{P}}=I\end{array}\right.$,

onde $P \in \Re, P \geq \bar{P}$ e $\alpha<0, I>0$ são números reais fixados. Vamos considerar o seguinte problema:

g) Provar que toda solução do sistema (5), $P(t)=P\left(t, 0, P_{0}\right), P_{0} \geq \bar{P}$, é periódica e estável.

Fixe $P_{0} \in \Re, P_{0}>\bar{P}$. Considere a solução do sistema (5) $P(t)=P\left(t, 0, P_{0}\right)$. Tal solução existe pelo Corolário 1 . O primeiro momento de salto $t=\theta_{1}^{0}$ pode ser encontrado a partir da equação:

$$
P_{0} e^{\alpha \theta_{1}^{\prime \prime}}=\bar{P}
$$

Assim, $\theta_{1}^{0}=\frac{1}{\alpha} \ln \frac{\bar{P}}{P_{0}}$. Desse modo, $P_{0}\left(\theta_{1}^{0}+\right)=\bar{P}+I$. O segundo momento de salto satisfaz a equação:

$$
(\bar{P}+I) e^{\alpha\left(\theta_{2}^{\prime \prime}-\theta_{1}^{\prime \prime}\right)}=\bar{P},
$$


Assim, $\quad \theta_{2}^{0}=\theta_{1}^{0}+\frac{1}{\alpha} \ln \frac{\bar{P}}{\bar{P}+I}$. Novamente, temos que $P_{0}\left(\theta_{2}^{0}+\right)=\bar{P}+I$.

Procedendo desta maneira, vamos encontrar que:

$$
\theta_{i+1}^{0}=\theta_{i}^{0}+\frac{1}{\alpha} \ln \frac{\bar{P}}{\bar{P}+I}
$$

para todo $i=1,2,3, \ldots$. Denote $\omega=\frac{1}{\alpha} \ln \frac{\bar{P}}{\bar{P}+I}$, então temos $i-1$ equações:

$$
\left\{\begin{array}{c}
\theta_{i}^{0}-\theta_{i-1}^{0}=w \\
\theta_{i-1}^{0}-\theta_{i-2}^{0}=w \\
\cdots \\
\theta_{2}^{0}-\theta_{1}^{0}=w
\end{array}\right.
$$

Se somarmos as equações acima, obtemos $\theta_{i}^{0}=\theta_{1}^{0}+(i-1) \omega$, e para todo $i=1,2,3, \ldots$, temos que

$$
P_{0}(t)=e^{-\alpha\left(t-\theta_{i}^{0}\right)}, \text { se } \theta_{i}^{0} \leq t \leq \theta_{\mathrm{i}+1}^{0} .
$$

Agora, temos que $\theta_{i}^{0} \leq t \leq \theta_{i+1}^{0}$ implica que $\theta_{i+1}^{0} \leq t+\omega \leq \theta_{i+2}^{0}$ e assim:

$$
P_{0}(t+\omega)=e^{-\alpha\left(t+\omega-\theta_{i+1}^{0}\right)}=e^{-\alpha\left(t+\omega-\left(\theta_{i}^{0}-\omega\right)\right)}=e^{\left(t-\theta_{i}^{\prime \prime}\right)}=P_{0}(t), \text { se } t \geq \theta_{0}^{1} .
$$

Assim, $P_{0}(t)$ é solução periódica do sistema (5).

Vamos agora investigar a estabilidade da solução do sistema (5). Para isso, vamos introduzir uma topologia no espaço das funções contínuas por partes $(P C)$.

Sejam $u(t), v(t):[0, \infty) \rightarrow \Re$ funções contínuas por partes e $\theta_{i}^{u}, \theta_{i}^{v}, i=1,2,3$, ..., seqüências estritamente crescentes dos momentos de descontinuidades de $u(t)$ e $v(t)$ respectivamente, $\operatorname{com} \theta_{i}^{u}, \theta_{i}^{v} \rightarrow \infty$ quando $i \rightarrow \infty$.

Dizemos que $u$ está em uma $\varepsilon$-vizinhança de $v$ se:

a) $\left|\theta_{i}^{u}-\theta_{i}^{v}\right|<\varepsilon, \forall i \geq 1$ 
b) $|u(t)-v(t)|<\varepsilon, t \in[0, \infty) \backslash \bigcup_{i=0}^{\infty}\left(\min \left\{\theta_{i}^{u}, \theta_{i}^{\prime \prime}\right\}-\varepsilon, \max \left\{\theta_{i}^{u}, \theta_{i}^{v}\right\}+\varepsilon\right)$.

Como visto, a solução $P_{0}(t)$ é estável se para um dado $\varepsilon>0$ existe um $\delta=\delta(\varepsilon)>0$ tal que $\left|P_{0}-P^{0}\right|<\delta$ implica que a solução $P(t)=P\left(t, 0, P^{0}\right), P^{0}>\bar{P}$ do sistema (5) está numa $\varepsilon$-vizinhança de $P_{0}(t)$.

Fixe $\varepsilon>0$ tal que $\varepsilon<\bar{P}+I$ e seja $\theta_{i}, i \geq 1$ a sequiência de pontos de descontinuidade de $P(t)$. Então, como $P_{0} e^{\alpha \theta_{1}^{0}}=\bar{P}$, temos:

$$
\begin{aligned}
& e^{\alpha \theta_{1}^{0}}=\frac{\bar{P}}{P_{0}} \\
& \alpha \theta_{1}^{0}=\ln \left(\frac{\bar{P}}{P_{0}}\right) \\
& \theta_{1}^{0}=\frac{1}{\alpha} \ln \left(\frac{\bar{P}}{P_{0}}\right)
\end{aligned}
$$

Do mesmo modo, $\theta_{1}=\frac{1}{\alpha} \ln \left(\frac{\bar{P}}{P^{0}}\right)$. Assim,

$$
\left|\theta_{1}-\theta_{1}^{0}\right|=\left|\theta_{1}^{0}-\theta_{1}\right|=\left|\frac{1}{\alpha} \ln \frac{\bar{P}}{P_{0}}-\frac{1}{\alpha} \ln \frac{\bar{P}}{P^{0}}\right|=\frac{1}{|\alpha|}\left|\ln \frac{P^{0}}{P_{0}}\right| .
$$

Assuma que $P^{0}>P_{0}>\bar{P}>0$. Assim, $\frac{P^{0}}{P_{0}}>1$ e deste modo:

$$
0<\ln \left(\frac{P^{0}}{P_{0}}\right)=\ln \left(1+\frac{P^{0}-P_{0}}{P_{0}}\right)<\frac{P^{0}-P_{0}}{P_{0}} \text {. }
$$

Logo,

$$
\frac{1}{|\alpha|}\left|\ln \frac{P^{0}}{P_{0}}\right|<\frac{1}{|\alpha|}\left(\frac{P^{0}-P_{0}}{P_{0}}\right)<\left(P^{0}-P_{0}\right) \frac{1}{|\alpha| \bar{P}} .
$$


Do modo como procedemos, escolhemos $\delta_{1}=\bar{P}|\alpha| \varepsilon$ e tal escolha implica que se $\left|P^{0}-P_{0}\right|<\delta_{1}$, então $\frac{1}{|\alpha|}\left|\ln \frac{P^{0}}{P_{0}}\right|<\varepsilon$.

Do mesmo modo, se $P_{0}>P^{0}>\bar{P}>0$, temos que $\frac{P_{0}}{P^{0}}>1$ e deste modo:

$$
0<\ln \left(\frac{P_{0}}{P^{0}}\right)=\ln \left(1+\frac{P_{0}-P^{0}}{P^{0}}\right)<\frac{P_{0}-P^{0}}{P^{0}} .
$$

Logo,

$$
\frac{1}{|\alpha|}\left|\ln \frac{P_{0}}{P^{0}}\right|<\frac{1}{|\alpha|}\left(\frac{P_{0}-P^{0}}{P^{0}}\right)<\left(P_{0}-P^{0}\right) \frac{1}{|\alpha| \bar{P}}
$$

Novamente, $\delta_{1}=\bar{P}|\alpha| \varepsilon$ o que implica que se $\left|P_{0}-P^{0}\right|<\delta_{1}$, então $\frac{1}{|\alpha|}\left|\ln \frac{P^{0}}{P_{0}}\right|<\varepsilon$.

Antes de prosseguir, vamos mostrar que se $x>0$, então $e^{x}-1 \geq 1-e^{-x}$, pois essa desigualdade será útil no que segue. De fato,

$$
\left(e^{x}-1\right)^{2} \geq 0 \Leftrightarrow e^{2 x}-2 e^{x}+1 \geq 0 \Leftrightarrow e^{2 x}-e^{x} \geq e^{x}-1 \Leftrightarrow e^{x}-1 \geq 1-e^{-x}>0
$$

Assuma, sem perda de generalidade, que $\theta_{1}<\theta_{1}^{0}$. Assim, $\theta_{i}<\theta_{i}^{0}$ para todo $i$ e $\theta_{i}^{0}-\theta_{i}=\theta_{1}^{0}-\theta_{1}<\mathcal{E}$. Se $t \in\left\lfloor\theta_{i}^{0}, \theta_{i+1}\right\rfloor$, então:

$\left|P_{0}(t)-P(t)\right|=\left|(\bar{P}+I) e^{\alpha\left(t-\theta_{i}^{0}\right)}-(\bar{P}+I) e^{\alpha\left(t-\theta_{i}\right)}\right|=(\bar{P}+I) e^{\alpha\left(t-\theta_{i}\right)}\left|e^{-\alpha\left(\theta_{i}^{0}-\theta_{t}\right)}-1\right|$.

Mas, $t>\theta_{i}$, pois $t \in\left\lfloor\theta_{i}^{0}, \theta_{i+1}\right\rfloor$ e $\theta_{i}<\theta_{i}^{0}$. Além disso, $e^{\alpha\left(t-\theta_{i}\right)}<1$. Logo,

$(\bar{P}+I) e^{\alpha\left(t-\theta_{i}\right)}\left|e^{-\alpha\left(\theta_{i}^{0}-\theta_{i}\right)}-1\right|<(\bar{P}+I)\left|e^{-\alpha\left(\theta_{i}^{0}-\theta_{i}\right)}-1\right|=(\bar{P}+I)\left|1-e^{-\alpha\left(\theta_{i}^{0}-\theta_{i}\right)}\right|$, pois $\alpha<0$.

Uma vez que $\theta_{i}^{0}-\theta_{i}<\varepsilon$, $(\bar{P}+I)\left|1-e^{-\alpha\left(\theta_{i}^{0}-\theta_{i}\right)}\right|<(\bar{P}+I)\left|1-e^{-\alpha \varepsilon}\right|$. Como $e^{\varepsilon|\alpha|}-1 \geq 1-e^{-\varepsilon|\alpha|}$, segue que: 
$(\bar{P}+I)\left|1-e^{-\alpha \varepsilon}\right|<(\bar{P}+I)\left|e^{\alpha \varepsilon}-1\right| \leq(\bar{P}+I)\left(1-e^{\alpha \varepsilon}\right)$.

Escolhemos agora $\delta_{2}=\frac{\varepsilon}{(\bar{P}+I)\left(1-e^{\alpha \varepsilon}\right)}$.

Finalmente, escolhemos $\delta=\delta(\varepsilon)=\min \left\{\delta_{1}, \delta_{2}\right\}$.Isso implica que:

$$
\left|P_{0}(t)-P(t)\right|<\varepsilon, t \in\left[\theta_{i}^{0}, \theta_{i+1}\right], i=1,2,3, \ldots
$$

Se $\left|P_{0}-P^{0}\right|<\delta$. Além disso, no intervalo $\left[0, \theta_{1}\right]$ temos que:

$$
\left|P_{0}(t)-P(t)\right|=\left|P^{0} e^{\alpha t}-P_{0} e^{\alpha t}\right|=e^{\alpha t}\left|P^{0}-P_{0}\right| \leq\left|P^{0}-P_{0}\right|<\delta
$$

Temos, então, que $P(t)$ está em uma $\varepsilon$-vizinhança de $P_{0}(t)$, se $\delta=\delta(\varepsilon)=\min \left\{\delta_{1}, \delta_{2}\right\}$. Isto implica que $P_{0}(t)$ é estável. 


\section{4 - Conclusões}

As Equações Diferenciais com Impulso (EDIs) têm importância em diversas aplicações na Física, na Economia e, em particular, na Medicina. Neste trabalho foram apresentados os principais resultados das EDIs no que diz respeito a existência de soluções e discutimos a estabilidade e a periodicidade das mesmas. Os Teoremas 1, 4 e 7 provam a existência de soluções das EDIs para determinadas condições.

A seguir, foi modelada a Pressão Arterial Sangüínea (PAS) usando a teoria de EDIs desenvolvida. Os problemas a) - g) apresentados no capítulo 3 mostram que o modelo proposto nos fornece, sob certas condições, soluções regulares, isto é estáveis e quase-periódicas (em alguns casos periódicas).

Acreditamos que esse modelo possa ser utilizado na investigação de arritmias e de outras anomalias cardíacas. 


\section{Referências Bibliográficas}

[1] AKHMET, M. U.; BEKMUKHAMBETOVA, G. A.; SERINAGAOGLU, Y.; The Dynamics of the Systemic Arterial Pressure Through Impulsive Differential Equations. http://www.iam.metu.edu.tr/research/preprint/34a.pdf, 1993.

[2] AKHMETOV, M.; PERESTYUK, N. A.; Periodic and Almost Periodic of Strongly Nonlinear Impulse Systems. Journal of Applied Mathematics, 56, 1992.

[3] BAINOV, D.; SIMEONOV, P.; Impulsive Differential Equations: Periodic Solutions and Applications. John Wiley \& Sons, New York, 1993.

[4] BOHR, Harald; Almost Periodic Functions. Chelsea Publishing Company, New York, 1947.

[5] BUCK, Robert C.; Advanced Calculus. McGraw-Hill, New York, 1978.

[6] CODDINGTON, Earl A.; LEVINSON, Norman.; Theory of Ordinary Differential Equations. McGraw-Hill, New York, 1955.

[7] COELHO, Flávio Ulhoa; LOURENÇO, Mary Lilian; Um Curso de Álgebra Linear. EDUSP, São Paulo, 2001.

[8] CORDUNEANU, C.; Almost Periodic Functions. Interscience Publishers, New York, 1968.

[9] HÖNIG, Chaim S.; Análise Funcional e Aplicações. IME/USP, São Paulo, 1970.

[10] HOPPENSTEADT, Frank. C.; Mathematical Aspects of Physiology - Lectures in Applied Mathematics, vol. 19. American Mathematical Society, Rhode Island, 1981.

[11] HOPPENSTEADT, Frank. C.; PESKIN, Charles S.; Mathematics in Medicine and Life Sciences. Springer-Verlag, New York, 1992. 
[12] PONTRYAGIN, L. S.; BOLTYANSKII, V. G.; GAMKRELIDZE, R. V.; MISHCHENKO, E. F.; The Mathematical Theory of Optimal Processes. John Wiley \& Sons, New York, 1962.

[13] SAMOILENKO, A. M.; PERESTYUK, N. A.; Impulsive Differential Equations. World Scientific, Singapura, 1995.

[14] SIMON, William; Mathematical Techniques for Biology and Medicine. Dover Publications, New York, 1986.

[15] SOTOMAYOR, Jorge; Lições de Equações Diferenciais Ordinárias. IMPA, Rio de Janeiro, 1979.

[16] RUDIN, Walter; Principles of Mathematical Analysis. McGraw-Hill, New York, 1976. 